\title{
Object Detection and Tracking for Creation of Interactive Videos
}

\author{
by \\ Jueya Li, B.Eng.

\begin{abstract}
A thesis submitted to the
Faculty of Graduate and Postdoctoral Affairs

in partial fulfillment of the requirements for the degree of
\end{abstract}

Master of Computer Science

Ottawa-Carleton Institute for Computer Science

The School of Computer Science

Carleton University

Ottawa, Ontario

November, 2017

(C) Copyright

Jueya Li, 2017 
The undersigned hereby recommends to the

Faculty of Graduate and Postdoctoral Affairs acceptance of the thesis

\title{
Object Detection and Tracking for Creation of Interactive Videos
}

\author{
submitted by Jueya Li, B.Eng. \\ in partial fulfillment of the requirements for the degree of \\ Master of Computer Science
}

Professor Oliver van Kaick, Thesis Supervisor

Professor Yuhong Guo, School of Computer Science

Professor Wonsook Lee, School of Electrical Engineering and Computer Science

Professor Doug Howe, Director, School of Computer Science

Ottawa-Carleton Institute for Computer Science

The School of Computer Science

Carleton University

November, 2017 


\section{Abstract}

Interactive videos can help to draw audiences' attention to certain content in a video, resulting in a more appealing or intriguing type of video. Object detection is a fundamental approach that can be used to automatically create interactive videos. In this thesis, we propose a new method for object detection based on combining object recognition with tracking in a neural network. Specifically, we use GoogLeNet [51] as a feature extractor, and then apply a long short-term memory (LSTM) network to further adjust the feature vectors extracted by GoogLeNet according to the context of the feature vectors extracted from the previous frame. We feed the output of the LSTM to a classifier and regressor as in the Overfeat network [46], to obtain predicted confidences and predicted bounding boxes. We pre-train the feature extractor on images of the ImageNet [29] and Pascal Visual Object Classes (VOC) [14] datasets. We then evaluate our network on the visual tracker benchmark OTB100 [5], which is a dataset composed of videos with objects from a variety of classes. We use this dataset for training and testing our network. We compare our results to results obtained without tracking, where GoogLeNet [51] is only combined with the Overfeat network [46] for classification and regression. Our model shows a better performance at predicting objects in frames where occlusion and background clutter appear, and results in more consistent object bounding boxes across frames. 


\section{Acknowledgments}

This thesis is in memory of my former supervisor, Dr. Anthony Whitehead. He gave me the basic idea for this research, which we started to work on together. He was such a great person and a helpful supervisor. He was always willing to help when I had any questions. May he rest in peace.

First of all, I would like to thank my supervisor, Dr. Oliver van Kaick, for helping me out in such a tough situation. He helped me with finding the research ideas for this thesis. He is also encouraging and inspiring when I face any problems.

Secondly, I would like to thank my parents for giving me financial support, so that I can conduct my research and pursue my dream here at Carleton University.

Thirdly, I sincerely thank the thesis committee for reviewing my work and giving me valuable suggestions.

In addition, I would like to thank all professors in the School of Computer Science. Thanking you for teaching me valuable knowledge and always being there to help. I would also like to thank the professors in the College of Information Technology for comforting me when I heard the sad news about my former supervisor.

At last, all the images and annotations we used in this paper come from ImageNet ILSVRC and Pascal VOC. We would like to thank all the contributors. 


\section{Table of Contents}

Abstract

Acknowledgments $\quad$ iv

Table of Contents $\quad$ v

List of Tables vii

List of Figures viii

1 Introduction 1

2 Background and related work $\quad 6$

2.1 Neural networks . . . . . . . . . . . . . . . . . 6

2.1.1 Inspiration from biology and the perceptron . . . . . . . 6

2.1.2 Architecture of modern neural networks . . . . . . . . . . . 9

2.1.3 Backpropagation algorithm ... . . . . . . . . . . 9

2.1.4 Commonly used activation functions . . . . . . . . . . . . . 9

2.2 Convolutional neural networks . . . . . . . . . . . . . . . . 13

2.2.1 Convolutional layer . . . . . . . . . . . . . . 17

2.2.2 ReLU layer . . . . . . . . . . . . . . . . . . . . 20

2.2.3 Pooling layer . . . . . . . . . . . . . . . . 20

2.2.4 Overfitting and dropout layer . . . . . . . . . . . . 20

2.3 Long Short-Term Memory (LSTM) network . . . . . . . . . . . . 22

2.4 Object detection and object tracking with neural networks . . . . . . 24

2.5 Implementation tools . . . . . . . . . . . . . . . . . . . 30

2.5.1 Tenforflow . . . . . . . . . . . . . . . 30

2.5.2 TF-Slim . . . . . . . . . . . . . . . . . . 30 
2.5.3 Optimizers used for learning . . . . . . . . . . . . . . . . 32

3 Object detection and tracking $\quad 34$

3.1 Overview . . . . . . . . . . . . . . . . . . . . 34

3.2 Architecture of the network . . . . . . . . . . . . . . 35

3.3 Pre-processing . . . . . . . . . . . . . . . . . . . 39

3.4 Training of the neural network . . . . . . . . . . . . . . . 39

3.5 Loss function . . . . . . . . . . . . . . . . . . . . . 41

3.6 Prediction . . . . . . . . . . . . . . . . . . . 43

3.7 Implementation notes . . . . . . . . . . . . . . . . . . . 43

4 Results $\quad 44$

4.1 Datasets . . . . . . . . . . . . . . . . . . 44

4.2 Results with video data. . . . . . . . . . . . . . . . 44

4.2.1 Results with our own data . . . . . . . . . . . . . 45

4.2 .2 Results with OTB videos . . . . . . . . . . . . . 55

4.3 Results with Image Data . . . . . . . . . . . . . . . . . . . . . 67

4.3.1 Results with Pascal VOC data . . . . . . . . . . . . . 67

4.3.2 Results with ImageNet Data . . . . . . . . . . . . . . . . . 70

$\begin{array}{lll}5 & \text { Conclusion } & 72\end{array}$

$\begin{array}{lll}6 & \text { Future Work } & 73\end{array}$

List of References $\quad 74$ 


\section{List of Tables}

2.1 Main Components available in TF-Slim . . . . . . . . . . . . . 32

4.1 Comparison of our work to the best-performing method mentioned in [52]. The evaluation measure is the mean Average Precision (mAP). . 68

4.2 The results of the ILSVRC2017 object detection task with provided

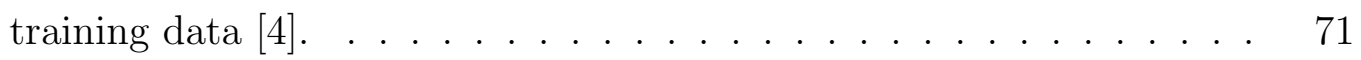




\section{List of Figures}

1.1 An example of an interactive video. Top: when the mouse cursor is moved over the beer bottle, the mouse glyph remains a pointer, which shows that the beer bottle is not clickable. Bottom: when the mouse cursor is moved over the soda can, its glyph becomes a hand, indicating that the soda can is the target object, which can be clicked by the user. Note that the object bounding boxes are shown for reference only and would not be visible in the video. . . . . . . . . . . . . . 2

2.1 Illustration of a biological Neuron. . . . . . . . . . . . . . . 7

2.2 Model of an artificial neuron (MP model). . . . . . . . . . . . . 8

2.3 Structure of the perceptron neural network, with learnable weights denoted in the neural connections. . . . . . . . . . . . . . 8

2.4 A 2-layer Neural Network (above) and a 3-layer Neural Network (below). 10

2.5 Plot of the sigmoid function. . . . . . . . . . . . . . . 12

2.6 Plot of the tanh function. . . . . . . . . . . . . . . . . . . 12

2.7 Plot of the ReLU function. . . . . . . . . . . . . . . . 13

2.8 Comparison of the tanh (dashed line) and ReLU (solid line) functions, showing the convergence rate of learning when using each function as the activation function of neurons. Image courtesy of Krizhevsky et al. [29]. . . . . . . . . . . . . . . . . . . . . . . . . 14

2.9 Interconnections between layers in the Neocognitron. Image Courtesy of Fukushima et al. [15]. . . . . . . . . . . . . . . . . . . . . 15

2.10 The shift invariant neural network model. Image courtesy of Zhang et. al [61]. . . . . . . . . . . . . . . . . . . . . . . . . 15

2.11 LeNet-5 neural network. Image courtesy of LeCun et al. [31] . . . . . 16

2.12 Convolutional pyramid. Image courtesy of Behnke et al. [11] . . . . . 17

2.13 Example of outputs generated from a $7 \times 7$ pixel input when the stride parameter is varied. When stride $=1$ (above); When stride $=2$ (below). 18 
2.14 Example of how a $7 \times 7$ input is modified with zero-padding. When the zero-padding is 2 , the input becomes $11 \times 11$. . . . . . . . . . 19

2.15 Example of max pooling with a $2 \times 2$ filter and stride of 2 . . . . . . 20

2.16 An example of overfitting: training data (left), and new test data (right). Please refer to the text for further explanation of the example. 21

2.17 A standard neural network (left) and a network with dropout regularization added (right). Only connections with a probability higher than a parameter $p$ are kept in the network. Image courtesy of Srivastava et al. [48]. . . . . . . . . . . . . . . . . . . . . . 22

2.18 Simple recurrent network. Image courtesy of Elman et al. [12] . . . . . 22

2.19 A Long Short-Term Memory cell. Image courtesy of Graves et al. [17]. 23

2.20 Naive Inception Module used in GoogLeNet. . . . . . . . . . . . . . . 25

2.21 Advanced Inception Module used in GoogLeNet. . . . . . . . . . . . . 26

2.22 Illustration of GoogLeNet with all layers included (continued in the next figure). Image courtesy of Szegedy et al. [51] . . . . . . . . . . . 27

2.23 Illustration of GoogLeNet with all layers included (continued from the previous figure). Image courtesy of Szegedy et al. [51]. . . . . . . . . 28

2.24 Example Python code (above) and its corresponding computation graph (below). Image courtesy of Abadi et al. [7]. . . . . . . . . . . . 31

2.25 Types of Operations Available in Tensorflow. Image courtesy of Abadi et al. [7]. . . . . . . . . . . . . . . . . . . . . .

3.1 The architecture of the ConvNet portion of our network. For each layer, we denote the type of unit, the number of units in the layer, the size of the image block processed by each unit, and the size of the output. 37

3.2 The architecture of the LSTM in our network. We denote the operations that are applied to the data before and after feeding it to the LSTM cells. . . . . . . . . . . . . . . . . . . . . 38

3.3 The Relative Position Formulas . . . . . . . . . . . . . . . . . . . . . 39

3.4 Training of the object detection neural network. . . . . . . . . . . . . 40

4.1 Frame 1 with predicted boxes (above) and hand-labeled boxes (below): 46

4.2 Frame 2 with predicted boxes (above) and hand-labeled boxes (below). 47

4.3 Frame 3 with predicted boxes (above) and hand-labeled boxes (below). 48

4.4 Frame 4 with predicted boxes (above) and hand-labeled boxes (below). 49

4.5 Frame 5 with predicted boxes (above) and hand-labeled boxes (below). 50 
4.6 Frame 6 with predicted boxes (above) and hand-labeled boxes (below). 51

4.7 Frame 7 with predicted boxes (above) and hand-labeled boxes (below). 52

4.8 Precision-Recall curves without LSTM and with LSTM . . . . . . . . 53

4.9 Result obtained with the network combined with the LSTM (below), compared to a network without this component (above). Note that the network with LSTM does not detect objects from other classes, e.g., the beer bottle. . . . . . . . . . . . . . . . .

4.10 Comparison of our results (above) to ROLO's result (below). Image courtesy of Ning et al. [40]. Our network detects several objects for each frame, while Ning's method only tracks one object in each video. 56

4.11 Training frame of the Bird class (above) and evaluation frame (below). The background of the frames in the training data is above the clouds, while the background in the evaluation frames is closer to the ground, where roofs and poles appear. . . . . . . . . . . . . . .

4.12 Detection results on some frames in a video clip of the Bird class with the LSTM (above) and without LSTM (below). The network with LSTM only detects one object incorrectly in each frame, while the network without LSTM detects two to three objects incorrectly in each frame. . . . . . . . . . . . . . . . . .

4.13 Precision-Recall curve for a video of the Bird class with LSTM and without LSTM. . . . . . . . . . . . . . . . . . .

4.14 An example of our network with LSTM successfully detecting and tracking the newly-appeared bird on the right of the frames. . . . . .

4.15 An example of our network with LSTM tracking the fading bird on the top-left, and removing the box when the bird fully disappears. . . . .

4.16 Training frame for the person class (left) and evaluation frame (right). The backgrounds of the two videos are different. There is occlusion in the evaluation frame, while there is none in the training frame. . . . .

4.17 Detection results on some frames of the Woman clip with LSTM (above) and without LSTM (below). The detections obtained by the network with LSTM are more consistent, especially for the frame in the middle.

4.18 Precision-Recall curves on the video clip Woman obtained with LSTM and without LSTM. . . . . . . . . . . . . . . . . . . 
4.19 Training frame of the Car class (above) and evaluation frame (below). The backgrounds of the frames are different, and there is scale variation in the evaluation frames. . . . . . . . . . . . . . . . . . 64

4.20 Detection results on frames of the video clip CarScale obtained with the LSTM (above) and without the LSTM (below). Both results are not satisfactory. The result obtained by the network with LSTM is slightly more accurate by avoiding the detection on the road given by the network without LSTM. . . . . . . . . . . . . . . .

4.21 Precision-Recall curve on the video clip Carscale with LSTM and without LSTM. . . . . . . . . . . . . . . . . . . . . . 66

4.22 Ground-truth boxes in a training frame. . . . . . . . . . . . . 66

4.23 Comparison of DetectorNet to other object detection works, in terms of mean Average Precision (mAP). Image courtesy of Szegedy et al. [52]. 68

4.24 Comparison of the Recall and Precision for the Bird class: our network (left), DetectorNet (right, image courtesy of Szegedy [52]). . . . . . . 70

4.25 Comparison of the Recall and Precision for the Bus class: our network (left), DetectorNet (right, image courtesy of Szegedy [52]). . . . . . . 70 


\section{Chapter 1}

\section{Introduction}

Hammoud describes interactive video as "a digitally enriched form of the original raw video sequence, allowing viewers attractive and powerful interactivity forms and navigational possibilities" [19]. The interactive videos that we aim to create are inspired by commercials on video streaming websites, e.g., YouTube. Commercials on YouTube provide static links placed at specific regions of the video screen. Then, if a viewer is interested in the product advertised in the video, he or she can click the link to be redirected to a page selling the product. However, this type of static link may not be appealing or intriguing enough for the audiences to click on. Thus, our goal is to make the objects that appear in the video clickable for greater interactivity. Such interactivity would be useful not only for commercials, but also for educational videos. For example, in a documentary video about wild animals in West Africa, by making the animals in the video clickable, the audiences would be able to click on the animal they are interested in to find more information about the animal species. An example of an interactive video is shown in Figure 1.1, where the soda can is the interactive object.

Our goal in this thesis is to provide a computational tool to facilitate the creation of such interactive videos. The creation of interactive videos can be roughly divided into two parts: object detection, and the inclusion of functionality for human computer interaction, e.g., clickable overlays added over the objects. In this thesis, we focus on the first task: object detection in videos. In practice, object detection is posed as the problem of detecting $2 \mathrm{D}$ bounding boxes that contain the objects.

To facilitate the creation of interactive videos and relieve users from manual work, 


\section{Interactive Video}

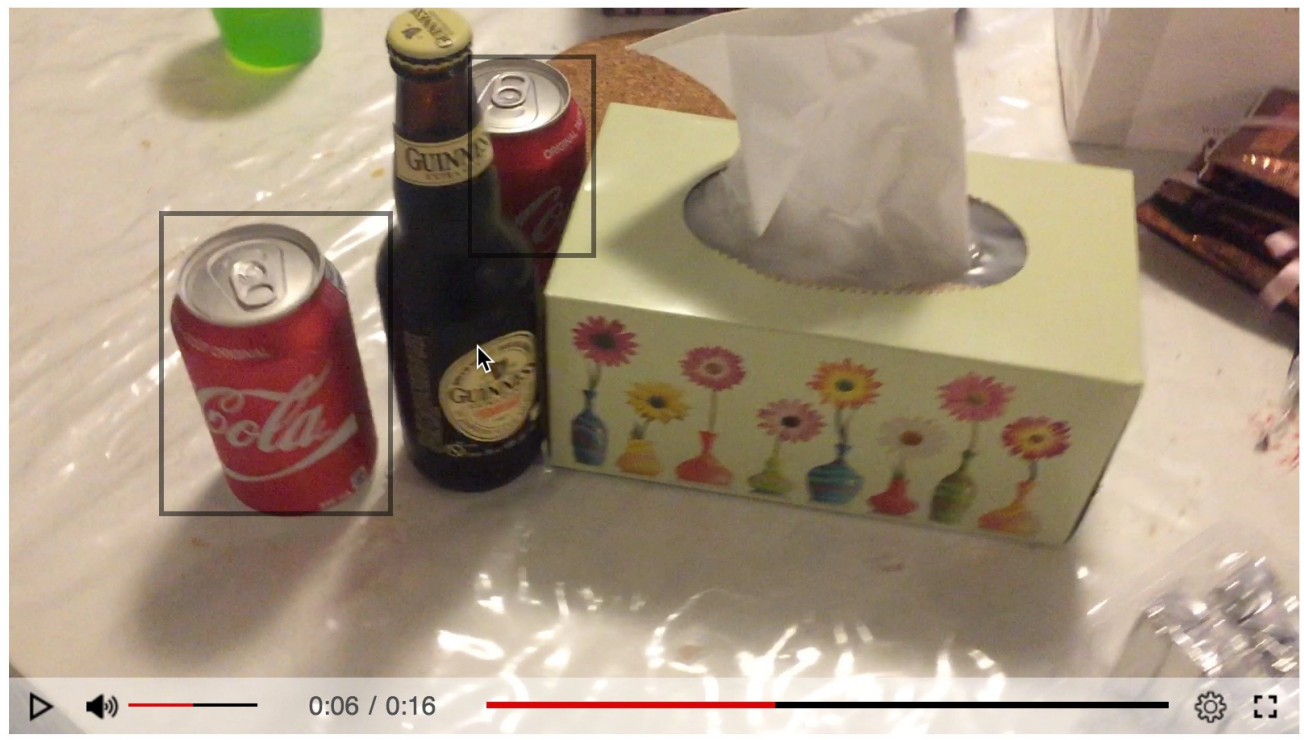

\section{Interactive Video}

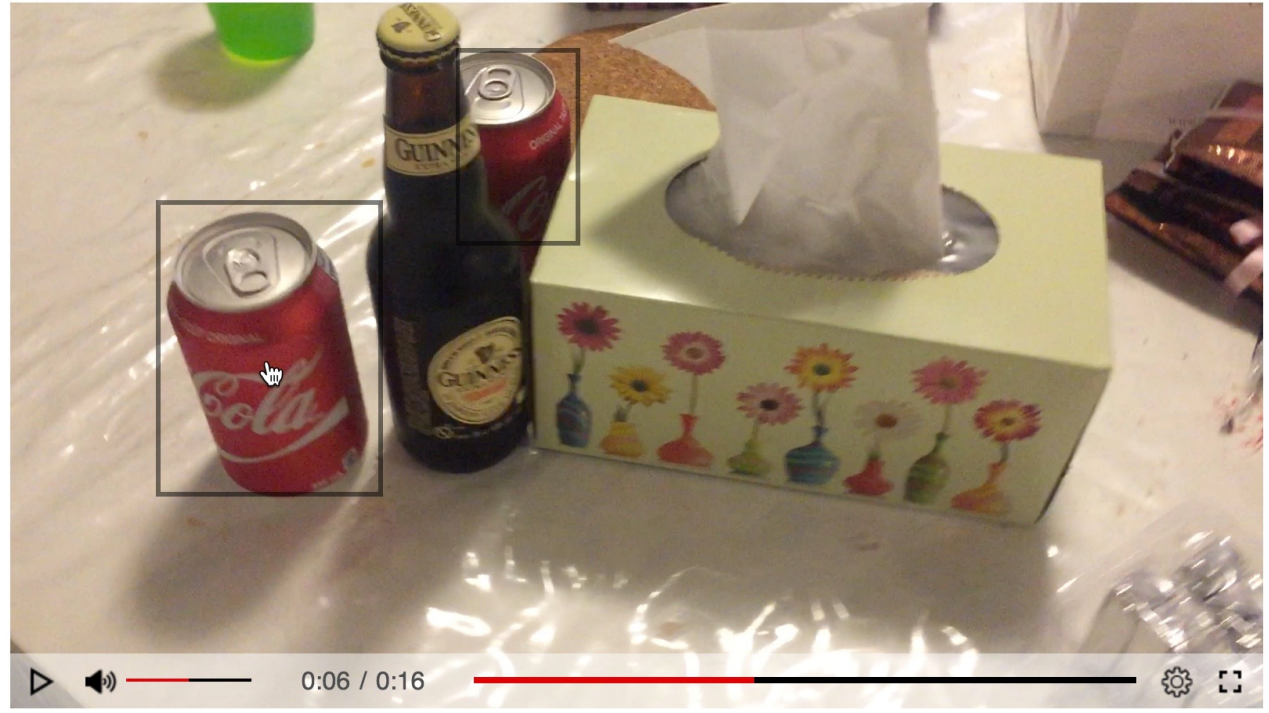

Figure 1.1: An example of an interactive video. Top: when the mouse cursor is moved over the beer bottle, the mouse glyph remains a pointer, which shows that the beer bottle is not clickable. Bottom: when the mouse cursor is moved over the soda can, its glyph becomes a hand, indicating that the soda can is the target object, which can be clicked by the user. Note that the object bounding boxes are shown for reference only and would not be visible in the video. 
a system that enables the detection of objects in a video should satisfy two main objectives: (i) The system should allow to detect a variety of objects from diverse classes; (ii) The detection should be highly accurate. To achieve these two objectives, we propose a system that performs both object detection and tracking. This combination can benefit the accuracy of object detection by taking into account temporal coherence and tracking objects across frames. Objects can be continuously detected even if they become partially occluded or are viewed at less favourable angles. Moreover, tracking can ensure the consistency of bounding boxes across frames, and can alleviate the problem of jitter in the bounding box locations. The accurate detection of bounding boxes will then reflect in a more satisfactory user experience when interacting with the videos.

To combine object detection with tracking, we propose a neural network that is a combination of a convolutional neural network commonly used for object detection in single images with a long short-term memory (LSTM) network. LSTMs are commonly used for speech recognition or sequence processing. In our work, we use this type of network for object tracking.

More specifically, we perform object detection and tracking by combining the GoogLeNet [51], LSTM [12,22], and Overfeat [46] networks. We use the convolutional architecture of GoogLeNet for feature extraction, to obtain feature vectors of video frames. Then, we feed the output of the GoogLeNet to the LSTM network, which functions as a tracker, adjusting the feature vector according to two consecutive frames of the video, based on a function learned with videos of tracked objects. At last, we use the structure proposed by Sermanet in the Overfeat [46] network, adding a classifier and regressor to get predicted confidences and bounding boxes from feature vectors.

The main benefit of this architecture is that it combines the best aspects of object detection and tracking. Temporal coherence is taken into account in the detection of objects in each frame, while at the same time, objects that newly appear in a frame can also be accounted for. A system based only on object detection in single images would not take advantage of temporal coherence, and would be susceptible to any problems arising in the detection of objects in individual frames. Moreover, a system performing a single round of object detection, followed by tracking with off-of-the-shelf methods, would not have the functionality of detecting new objects as they appear in the video. 
Moreover, smoothing could also be used to avoid jitter in the bounding boxes of detected objects. The difference between smoothing and tracking is that a tracking method learns the correlations between two successive frames, while smoothing is applied locally and mainly eliminates noise. In our approach, when tracking an object, we provide the features extracted by the convolutional network both for the last frame and current frame as the input of the LSTM. The LSTM then learns the correlations between the two frames, and adjusts the current feature map according to the correlation. The bounding boxes and the confidences of the bounding boxes are therefore corrected as well.

We pre-train our network on images from the ImageNet Large Scale Visual Recognition Competition (ILSVRC) and the Pascal Visual Object Classes (VOC) dataset, which are benchmarks in visual recognition. The Pascal VOC data provides images of 20 different object classes, while the ILSVRC provides images for objects or 1,000 classes. As part of our evaluation, we first verified that our method yields state-of-art accuracy for detecting objects in single images of these datasets.

Moreover, to evaluate our method with suitable videos that can be made interactive, we use the Visual Tracker Benchmark OTB100 [5], and two self-recorded videos. OTB100 [5] is a dataset with 100 video clips intended for evaluating visual trackers. We chose 30 of them and performed qualitative evaluations comparing to a network without LSTM tracker. The self-recorded videos are videos that we recorded for intentionally adding occlusions, scale variations, and illumination variations.

We found with a qualitative analysis of the results that our proposed network with the LSTM component has a better accuracy for object detection on frames where occlusions and background clutter occur. We also performed a quantitative evaluation for comparing the performance of our network with a version of the network that does not perform tracking. We found that our network outperformed the network without tracking in all the experiments. It improved the Average Precision of detection from $1 \%$ to $10 \%$, when feature extraction performed well. More impressively, when feature extraction was unsatisfactory due to bad training data, the tracking improved the Average Precision by $80 \%$.

Finally, we find that our method is relatively efficient, processing 6 frames per second on a modern CPU - a desktop with an Intel Core $i 7-7,7003.60 G \mathrm{~Hz} \times 8$ CPU, with 223.6 GB of memory and a GeForce GTX 1050 GPU.

To summarize, the main contribution of this thesis is a new network for object 
detection and tracking, which combines a convolutional network for object detection in single frames with an LSTM network for tracking objects across multiple frames. We show that this leads to more satisfactory results when detecting objects in videos of different categories.

The remainder of this thesis is organized as follows. In Chapter 2, we provide some background on neural networks and discuss works related to ours; In Chapter 3, we describe our network architecture in more detail; In Chapter 4, we evaluate our network with experimental results, and also compare our work with selected related works; In Chapter 5, we conclude the thesis and discuss future works. 


\section{Chapter 2}

\section{Background and related work}

In this chapter, we first provide some background on neural networks, and then discuss their use for object detection and tracking. We finalize with a discussion of the software libraries that we use for implementing our method.

\section{$2.1 \quad$ Neural networks}

\subsubsection{Inspiration from biology and the perceptron}

Neurons are the basic computational units of our brains. Each neuron has several dendrites and an axon [33]. Neurons receive input signals from dendrites and send output signals through their axons. The axons then connect to dendrites of other neurons via synapses. Figure 2.1 provides an illustration of a biological neuron.

In 1943, McCulloch and Pitts [36] created a mathematical model of a biological neural network (MP model), effectively proposing the first Artificial Neural Network (ANN). The goal of this model was to solve problems that are hard to solve with rule-based programming algorithms [23]. In this thesis, we will call ANNs as neural networks (NNs) for short.

Figure 2.2 shows an illustration of an artificial neuron (MP model). If we let the input signal from another neuron at a synapse be $x_{i}$, and the synaptic strength at the synapse be $w_{i}$, then the signal from this neuron is modulated by the synaptic strength: $\left(w_{0} x_{0}\right)$. The synaptic strengths, also known as the weights of the neural network, are learnable. They control the strength and the direction of the influence that one neuron has on another. For one neuron, all the inputs signals that its dendrites receive are summed up. The neuron fires and send a signal via its axon if 
the calculated sum is above a predefined threshold. In the mathematical model, we only consider the frequency of firing, which we model as an activation function $f$.

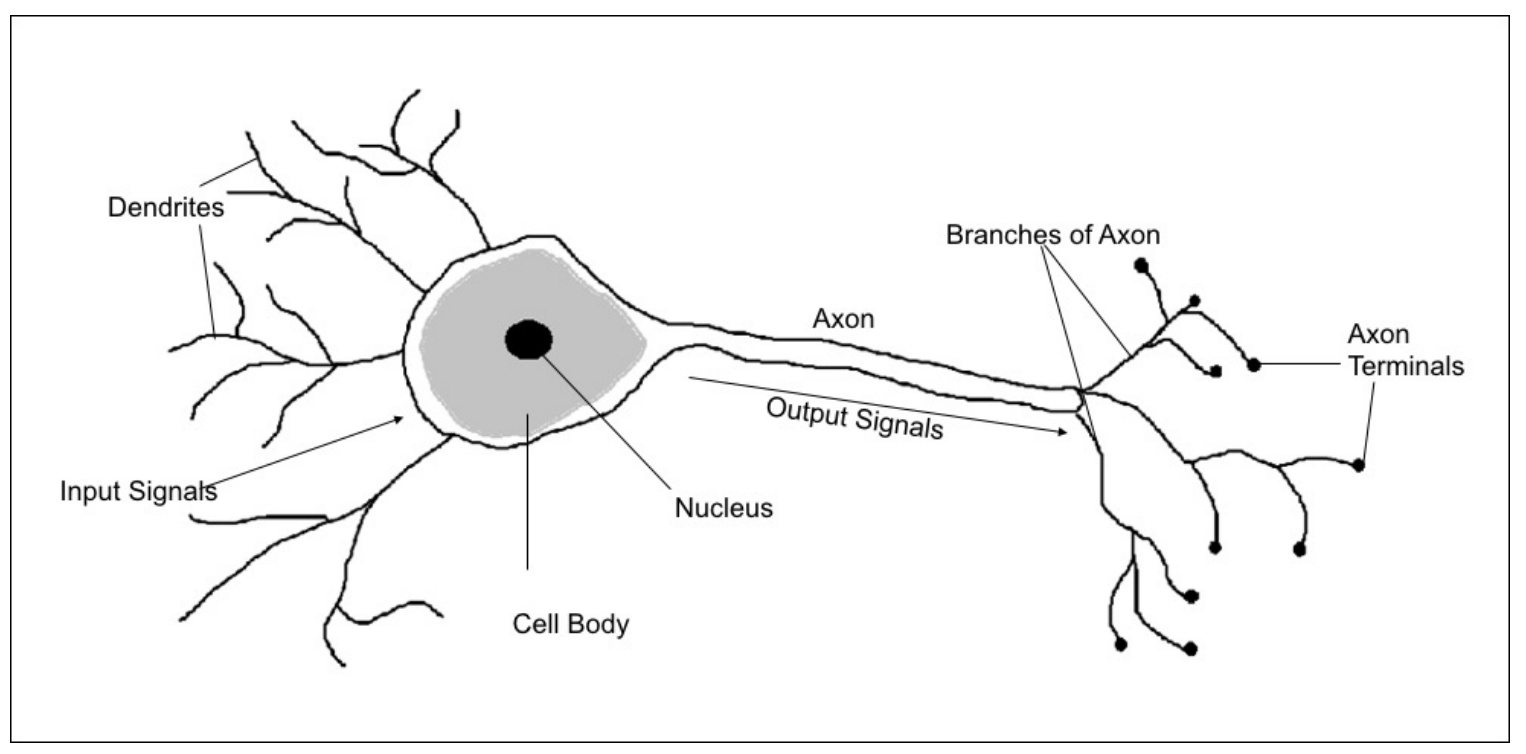

Figure 2.1: Illustration of a biological Neuron.

In the MP model of McCulloch and Pitts [36], all the weight values were predefined. Thus, the weights could not be learned. After that, in 1949, Hebb [20] introduced Hebbian learning, believing that the synaptic strength at synapses is changeable. Based on this theory, research started on learning functions with neural networks by adjusting the weights. In 1958, Rosenblatt [43] created the perceptron, a neural network consisting of two layers of neurons: an input layer and an output layer. It was the first artificial neural network that could learn. Only the neurons in the output layer perform calculations, whereas input units are only responsible for data transmission. The architecture of the perceptron is shown in Figure 2.3.

The perceptron is only capable of solving simple linear classification problems. In 1969, Minsky et al. [38] demonstrated the disadvantages of the perceptron structure, showing that the perceptron is not capable of solving exclusive-or problems. They also argued that, if the number of layers in the network were increased to account for this shortcoming, the amount of computation required for learning would be too large to be handled by computers of the time. 




Figure 2.2: Model of an artificial neuron (MP model).

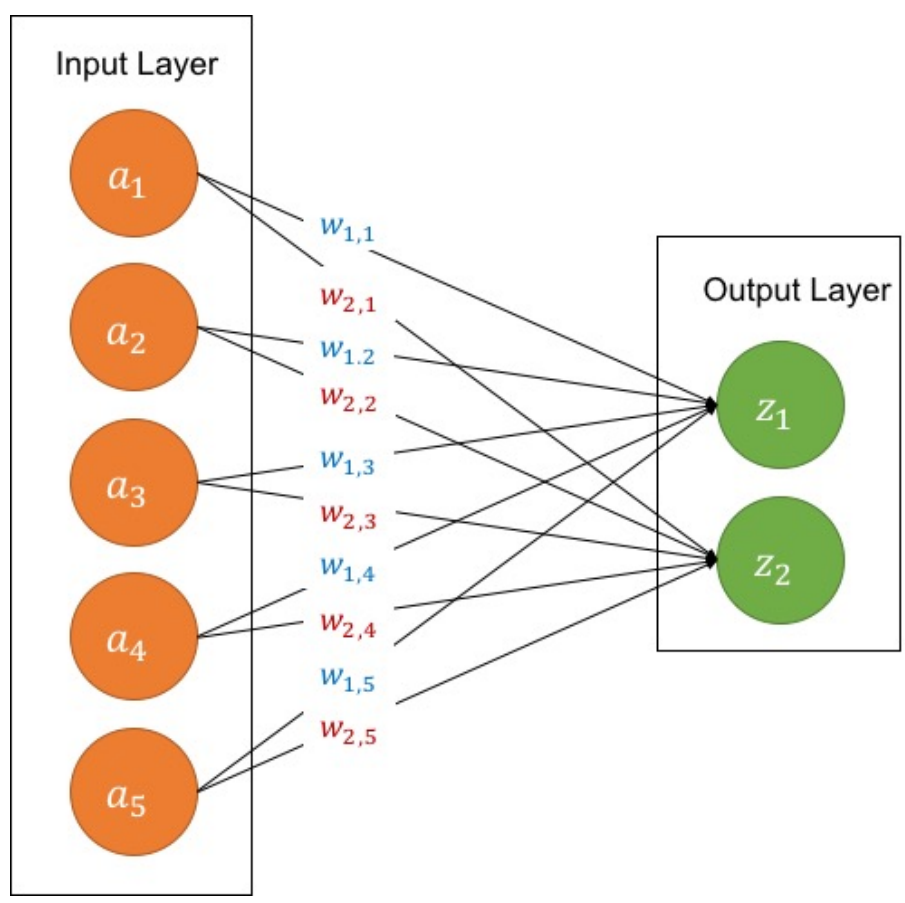

Figure 2.3: Structure of the perceptron neural network, with learnable weights denoted in the neural connections. 


\subsubsection{Architecture of modern neural networks}

More contemporary neural networks are composed of an input layer and an output layer, in addition to 0 to $n$ hidden layers in between. The hidden layers are most commonly fully-connected layers. In the output layer, neurons normally do not trigger an activation function because the output layer is usually taken to represent the result of the processing in the form of real-valued numbers (in classification), or real-valued targets (in regression). Figure 2.4 shows two examples of neural network topologies.

\subsubsection{Backpropagation algorithm}

In 1975, Werbos [57] described in his thesis that the backpropagation algorithm can be used for training neural networks. Moreover, in 1986, Rumelhart and McClelland conducted experiments showing that the backpropagation algorithm can be used to generate inputs in hidden layers.

There are two phases in backpropagation. The first is the propagation phase, consisting in two propagation steps. First, the forward propagation of the inputs through the NN, to obtain all the outputs. Second, the backward propagation of the outputs to generate deltas for each hidden neuron. When using the delta rule [35], we calculate the differences between the predicted values (outputs) and the required values, which are called the deltas. The second phase of backpropagation is the update of the weights according to the deltas. For a neuron in the output layer or hidden layer, we multiply its input with delta, and calculate its gradient. Then we take the negative value of a percentage of the gradient, and add it to the neuron's weight [8]. This ratio or percentage will influence the speed and performance of training; thus, it is called the learning rate. The direction of the gradient indicates the direction that the loss increases; thus, we take the negative of the gradient to decrease the loss caused by the weight.

\subsubsection{Commonly used activation functions}

Activation functions are used to adjust the range of numbers generated by the neural network. Sigmoid, tanh, and ReLU are three of the most commonly used activation functions. 

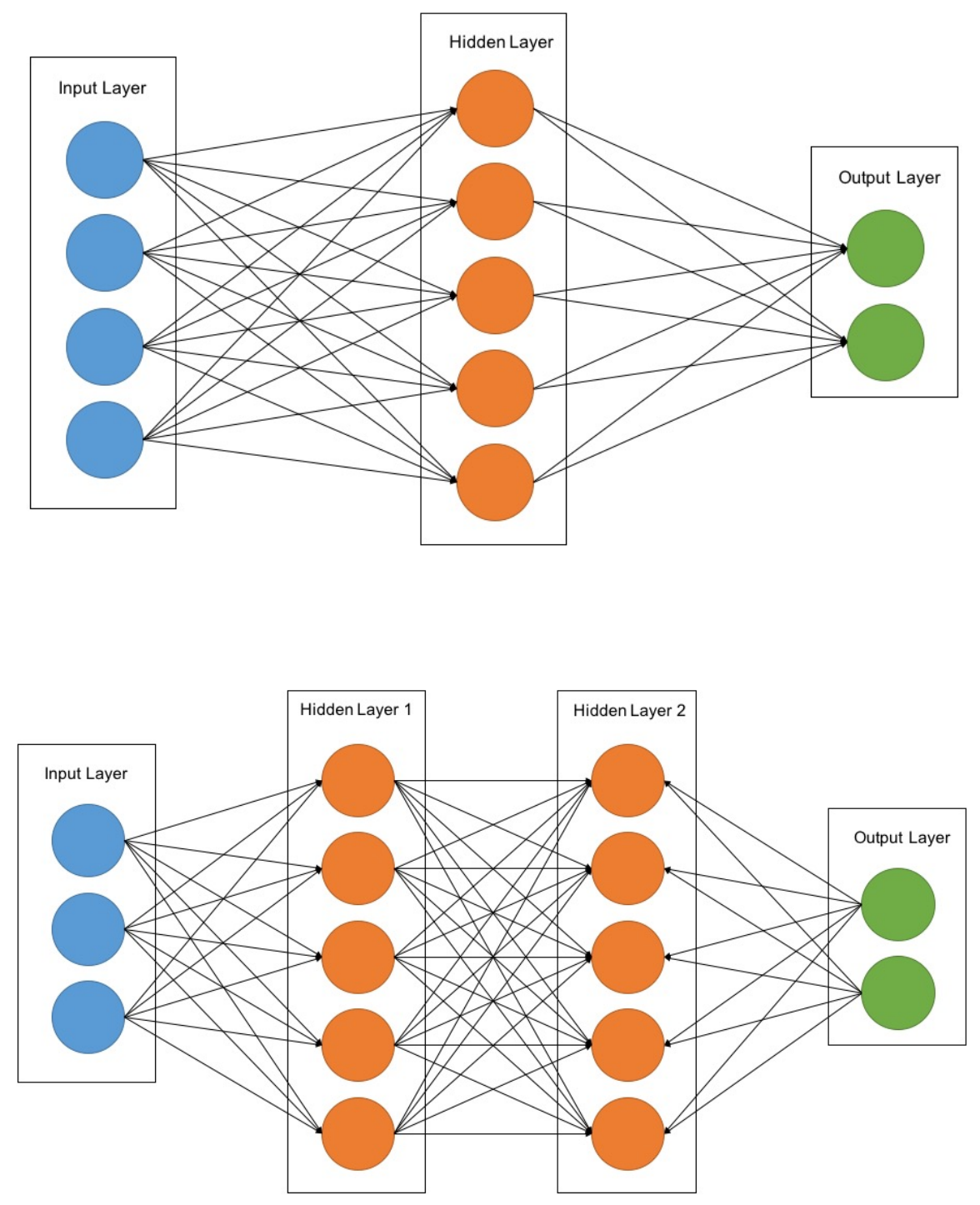

Figure 2.4: A 2-layer Neural Network (above) and a 3-layer Neural Network (below). 
The mathematical representation of a sigmoid function is given as:

$$
\sigma(x)=1 /\left(1+e^{-x}\right),
$$

where $x$ is the input of neurons, the $w_{i} x_{i}$ illustrated in Figure 2.2. The sigmoid function normalizes its real-valued inputs to a number between 0 and 1 . Large negative numbers become 0 , while large positive numbers become 1 . Figure 2.5 illustrates the sigmoid function.

The sigmoid function has been rarely used in neural networks due to its two drawbacks:

- The sigmoid function can cause the gradient to vanish or explode. The local gradient of the current neuron is the delta between the output of the neuron (neuron activation) and our expected output of the neuron. The output gradient is the delta between the output of the network and our expected output of the network. When neuron activations reach either 0 or 1 , the local gradients become almost zero. Now, recall that, during backpropagation, the local gradients are multiplied with the output gradients. Thus, the gradients vanish. Otherwise, if the initial weights are too large, then the gradient will become saturated.

- The outputs of the sigmoid function are not zero-centered. If the input data is always positive, then the gradients are either all positive, or all negative. Adding up all the gradients can remedy this issue to some extent. Thus, this drawback is less severe than the previous one.

Moreover, the tanh function (hyperbolic tangent) is used to normalize a real number to the range between -1 and 1 . It is defined as:

$$
\tanh (x)=2 \sigma(2 x)-1 .
$$

The outputs of the tanh function are zero-centered, thus preferred over the sigmoid function. Figure 2.6 illustrates the tanh function.

Finally, the ReLU function, short for Rectified Linear Unit, is defined as:

$$
f(x)=\max (0, x) .
$$




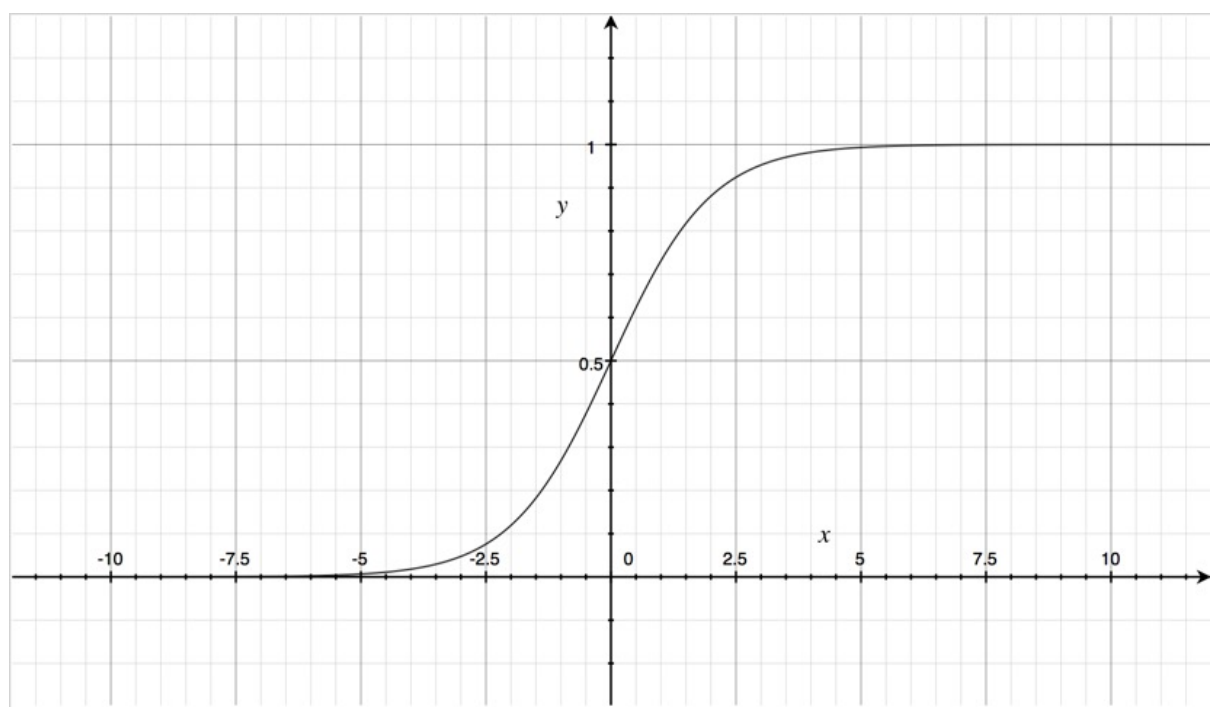

Figure 2.5: Plot of the sigmoid function.

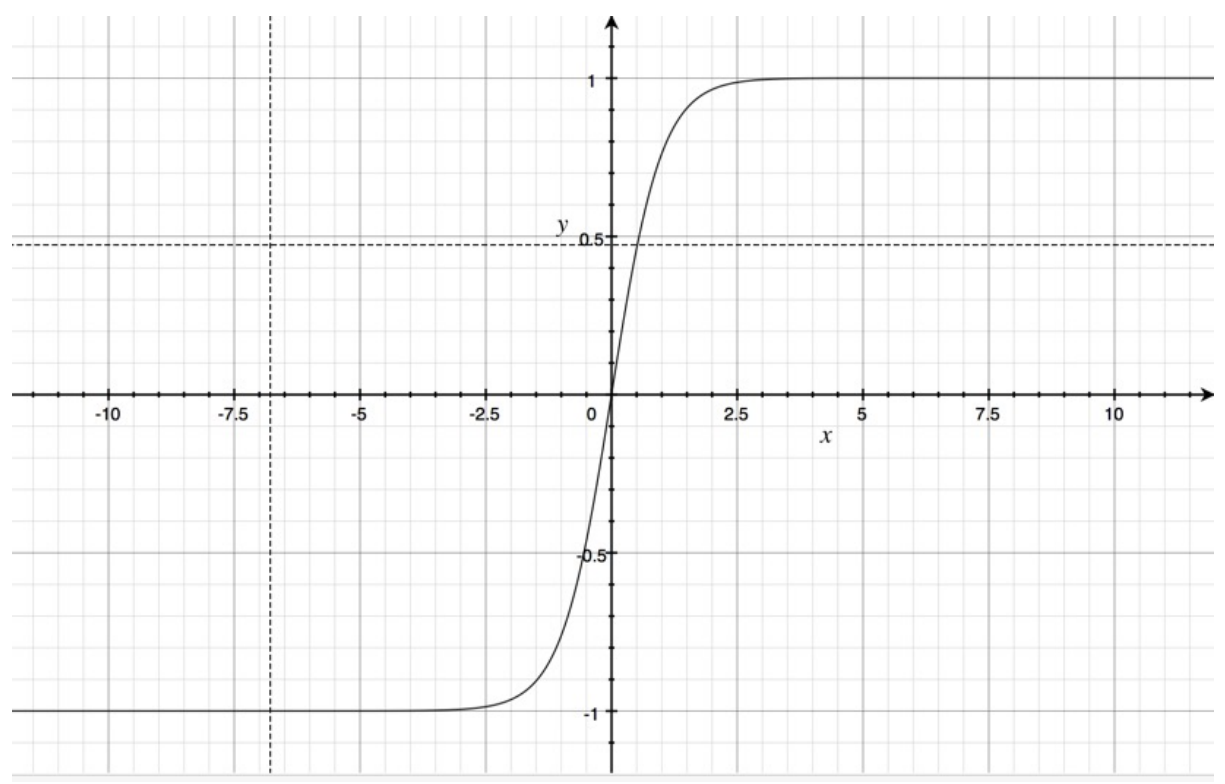

Figure 2.6: Plot of the tanh function. 
Figure 2.7 illustrates the ReLU function. The ReLU function has two major advantages: (i) It can greatly accelerate the convergence of stochastic gradient descent (SGD). It does not require expensive mathematical operations, e.g., exponentials. Figure 2.8 presents an experiment by Krizhevsky et al. [29] that demonstrates how the convergence of learning when using ReLU functions is six times faster than when the tanh function is used.

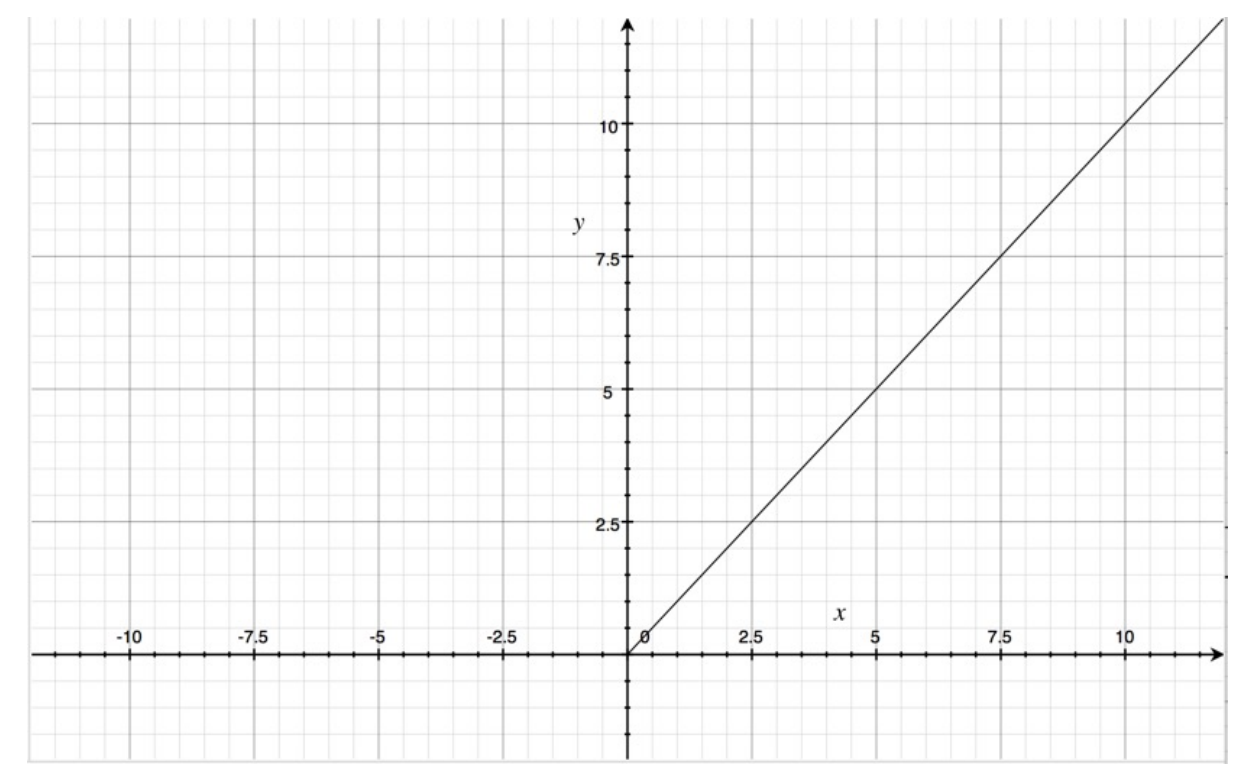

Figure 2.7: Plot of the ReLU function.

The ReLU function has one disadvantage. Neurons using ReLU activation functions are "fragile", meaning that they can become inactive during training. As explained in [30], when most inputs to ReLU units are positive, ReLU gates will remain open, gradients will enable backpropagation, and the inputs of ReLU units will be updated. When the learning rate is high, the updates on the inputs will be larger, which may cause most of the inputs to become negative. ReLU gates will then be closed.

\subsection{Convolutional neural networks}

Convolutional neural networks (CNNs, or ConvNets) are deep and feed-forward neural networks that are normally used in image analysis and classification [55]. In 1962, 


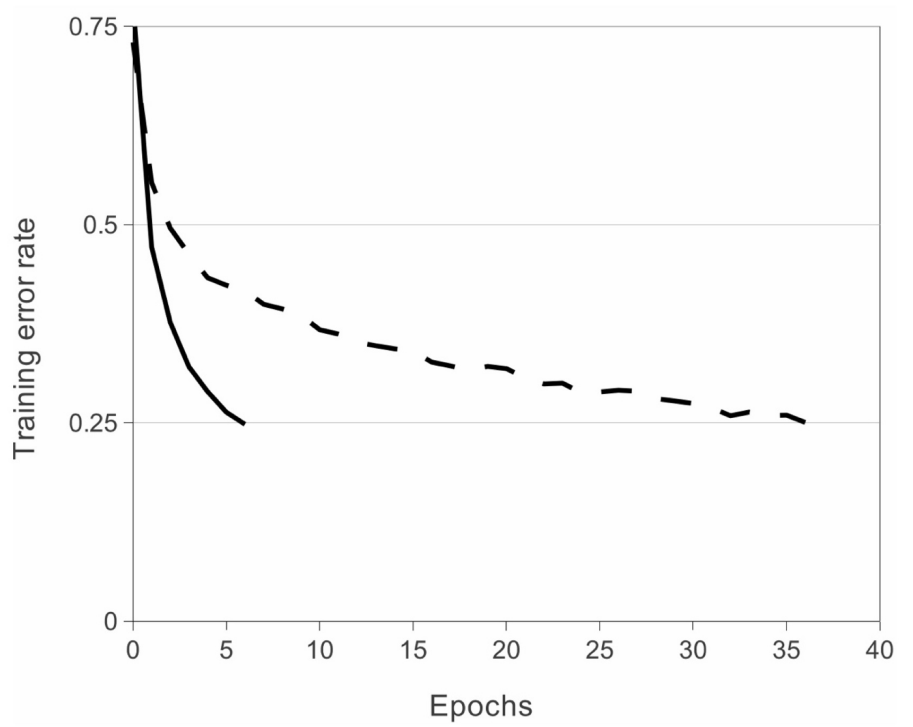

Figure 2.8: Comparison of the tanh (dashed line) and ReLU (solid line) functions, showing the convergence rate of learning when using each function as the activation function of neurons. Image courtesy of Krizhevsky et al. [29].

Hubel et al. [24] studied the visual cortex of cats and monkeys, proposing the existence of a structure in the eyes of these animals called the receptive field. Visual stimuli in small regions of the visual field trigger the firing of only one neuron in the receptive field. The neighbors of this neuron have similar receptive fields. Their study also proposed the existence of two types of visual cells: simple cells, and complex cells. Simple cells detect straight edges with specific directions, whereas complex cells do not take the position of edges into consideration [55].

Based on these observations, Fukushima and Kunihiko [15] introduced the Neocognitron. The Neocognitron was the first implemented convolutional neural network and the first application of receptive field theory to artificial neural networks. The Neocognitron divides a visual pattern into many sub-patterns (features). Then, all the features go through a cascade of feature planes. The Neocognitron models biological visual systems, thus, enabling recognition even when objects are shifted or slightly twisted. Figure 2.9 is an illustration showing the interconnections between layers in the Neocognitron [15].

Zhang et al. [60,61] proposed a similar shift-invariant neural network model, trained with backpropagation. The architecture of this model is shown in Figure 2.10. 


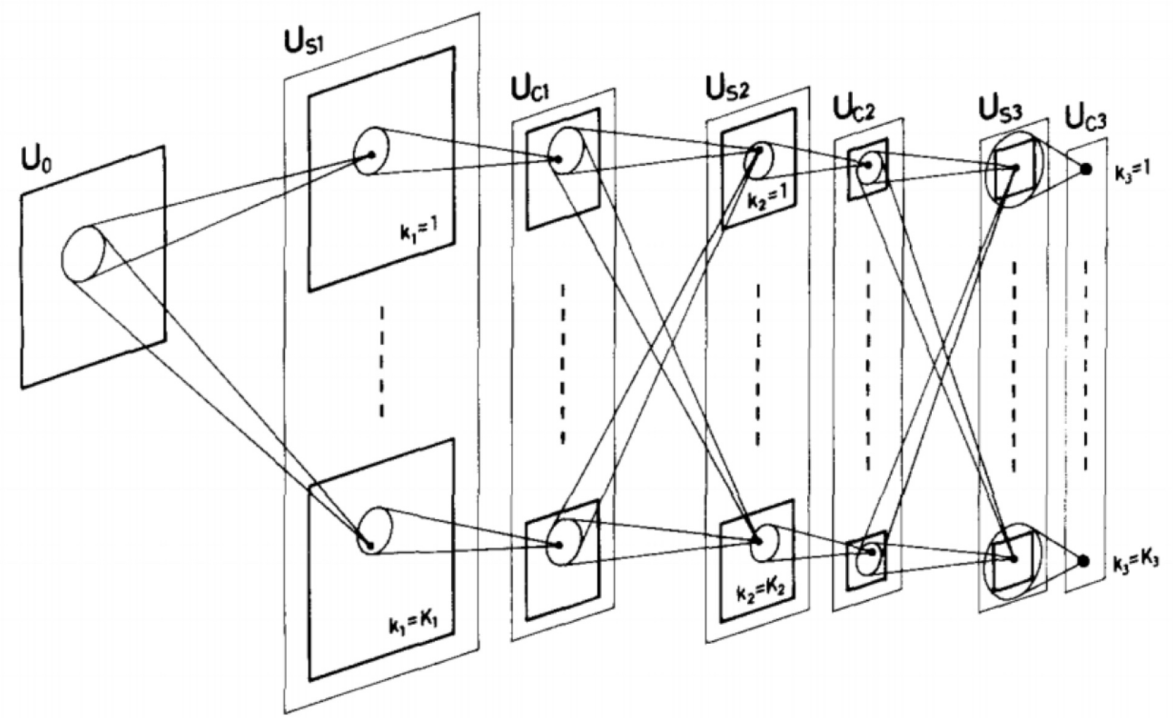

Figure 2.9: Interconnections between layers in the Neocognitron. Image Courtesy of Fukushima et al. [15].

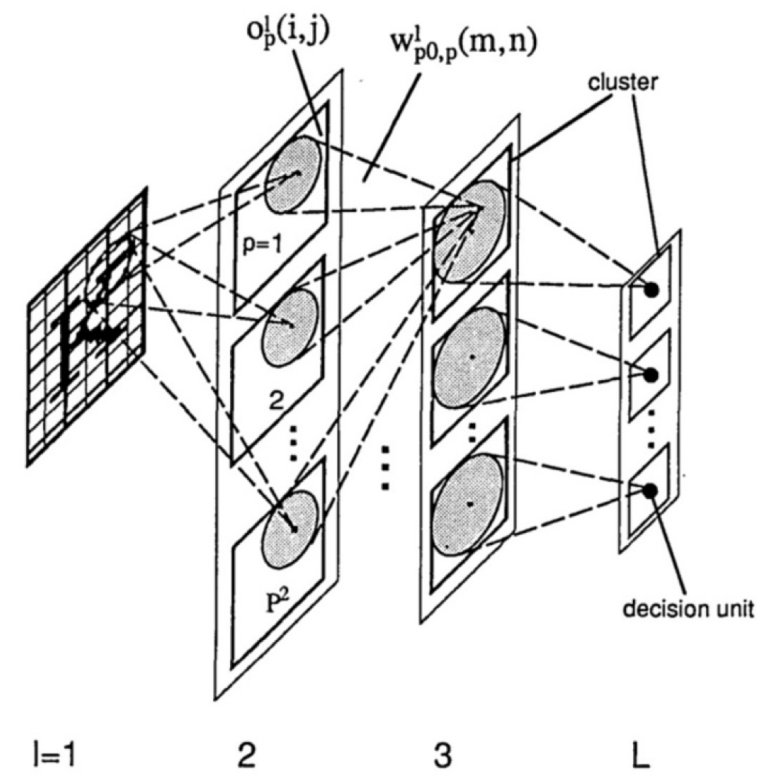

Figure 2.10: The shift invariant neural network model. Image courtesy of Zhang et. al [61]. 
Moreover, LeCun et al. [31] constructed a 7-level convolutional neural network called LeNet-5 to recognize hand-written digits. Figure 2.11 shows the architecture of LeNet. There are three types of layers in this neural network: convolutional layer, subsampling layer, and fully-connected layer. All the convolutional layers perform convolution with a kernel of size $5 \times 5$, and all the units in one feature map share the same kernel. Kernels are displaced by one pixel. Subsampling layers use a $2 \times 2$ kernel, where the kernel is displaced by two pixels, so that the input fields do not overlap.

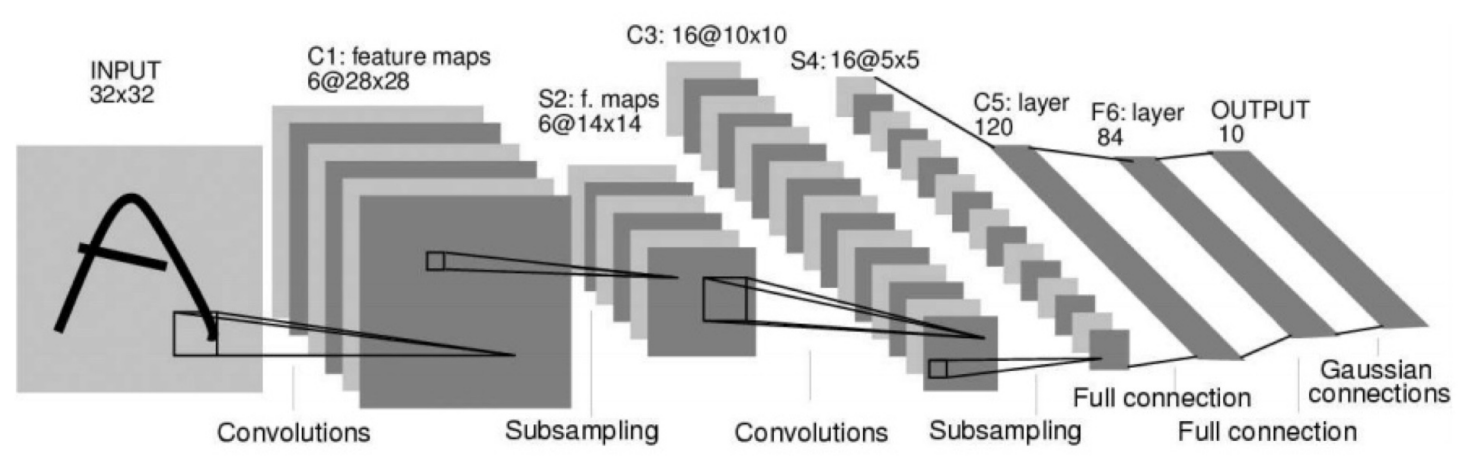

Figure 2.11: LeNet-5 neural network. Image courtesy of LeCun et al. [31].

Modern convolutional networks have been successfully used in image classification problems, since it is difficult for regular neural networks to handle such problems. For example, a network such as CIFAR-10 [1], which takes as input images of size $32 \times 32 \times 3$, requires 3,072 weights to define its fully-connected layer. Training such networks with fully-connected layers can be an expensive procedure.

Each hidden layer of a convolutional neural network can be abstracted as having three dimensions: width, height, and depth. The architecture of the network can be represented by a convolutional pyramid [11], as shown in Figure 2.12. The width and height are the dimensions of the spatial information, whereas the depth can be seen as the amount of semantic information that is extracted. Convolutional networks are commonly composed of three types of layers: convolutional layers, pooling layers, and fully-connected layers. For example, the CIFAR-10 network [28], used for image classification, possesses the following architecture: input layer - convolutional layer ReLU layer - pooling layer - fully-connected layer. 


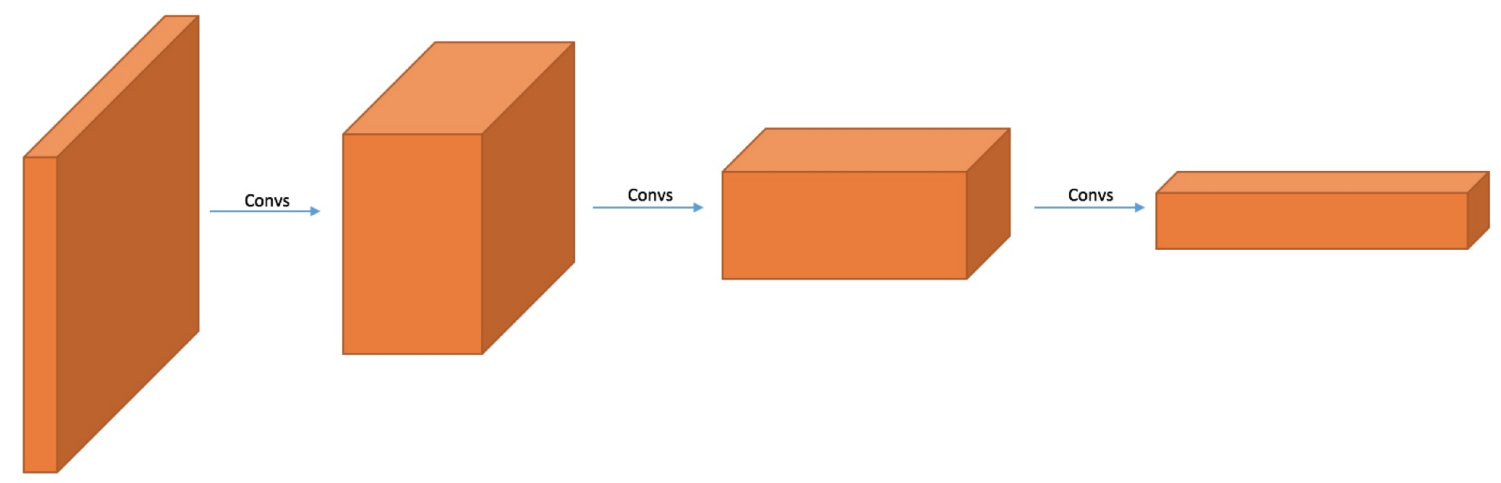

Figure 2.12: Convolutional pyramid. Image courtesy of Behnke et al. [11].

\subsubsection{Convolutional layer}

The convolutional layer is the most critical part of convolutional neural network. The parameters of convolutional layers are a set of filters, also called kernels. Each kernel can be seen as sliding through the input image and computing the dot product of the kernel and the input portion of the image that it covers. After sliding through the whole image, the convolution provides a 2-dimensional feature map. Each kernel provides one feature map. Thus, we obtain the same number of feature maps as kernels in the convolutional layer. The output of this layers is composed of all the feature maps stacked together.

Three factors control the size of the output: depth, stride, and zero-padding. The depth is a hyperparameter determined by the number of kernels. The stride is how many pixels the kernel moves, when sliding though an image. Figure 2.13 shows an example of how different strides provide outputs of different sizes, given an input of $7 \times 7$ pixels with a kernel of size $3 \times 3$.

Another hyperparameter is the zero-padding. Zero-padding is normally used to preserve the spatial size of the input. Figure 2.14 shows an example of how the zero-padding changes the input size for a $7 \times 7$ input.

The width of the output of the convolutional layer is given by:

$$
W_{\text {output }}=\frac{(W-K+2 P)}{S}+1,
$$


$7 \times 7$ Input Volume

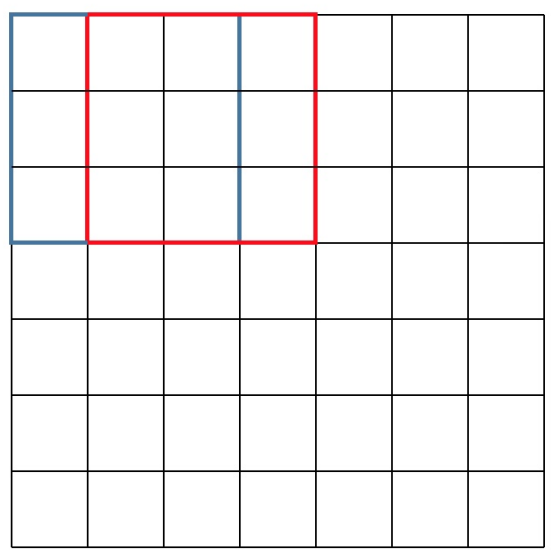

$7 \times 7$ Input Volume

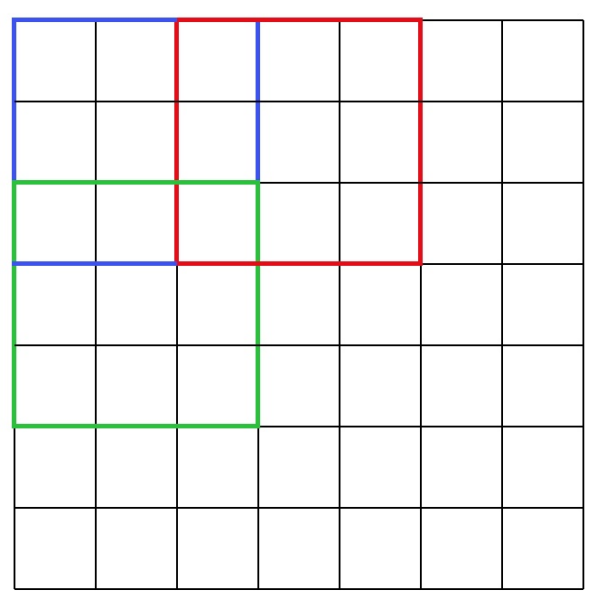

$5 \times 5$ Output Volume

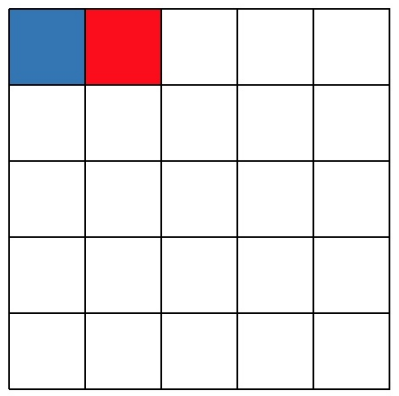

$3 \times 3$ Output Volume

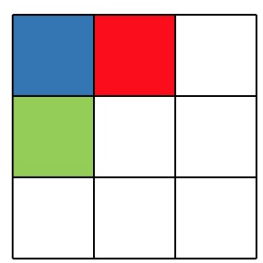

Figure 2.13: Example of outputs generated from a $7 \times 7$ pixel input when the stride parameter is varied. When stride $=1$ (above); When stride $=2$ (below) 


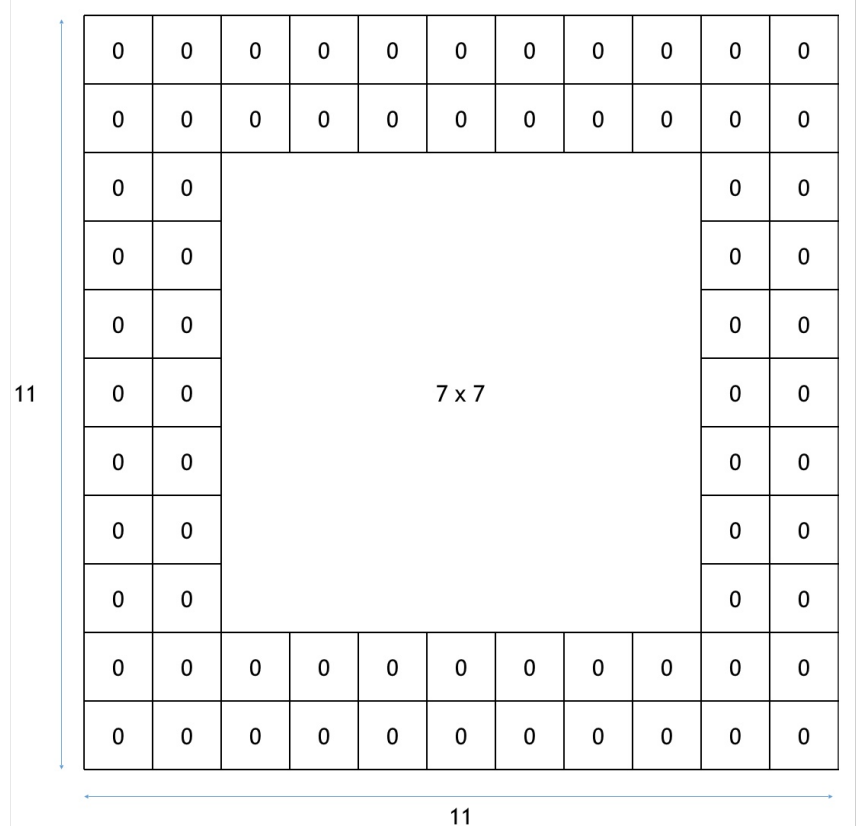

Figure 2.14: Example of how a $7 \times 7$ input is modified with zero-padding. When the zero-padding is 2 , the input becomes $11 \times 11$.

where $W$ is the input width, $K$ the kernel width, $S$ the stride, and $P$ the padding. The output height can be computed in a similar manner.

Parameter sharing is used in convolutional neural networks, as promoted by Zhang [61], which reduces the parameters that need to be trained in the network. For each feature map, we constrain all the neurons to share the same weights and bias. Therefore, after calculating the gradients for each neuron in one feature map, the gradients are added together and used to update the weights when performing backpropagation [2].

Yu and Koltun [58] introduce a hyperparameter called dilation. It is applied to kernels to introduce spacing between image pixels. In this way, each neuron has a larger receptive field, and the spatial data is merged more quickly. 


\subsubsection{ReLU layer}

ReLU layers are normally applied after each convolutional layer. Its usage is the same as the use of activation functions in fully-connected layers. Hinton et al. [39] find that a ReLU function works better that a sigmoid or tanh function, because it does not influence the accuracy significantly, while speeding up the training. ReLU layers are used to increase nonlinear properties in the convolutional network.

\subsubsection{Pooling layer}

The function of pooling layers is to reduce the spatial volume of the input, reduce the number of parameters, and control overfitting [55]. The most commonly used method is max pooling. That is, the pooling returns as output the maximum value of the region covered by the filter. Figure 2.15 illustrates the max pooling function.

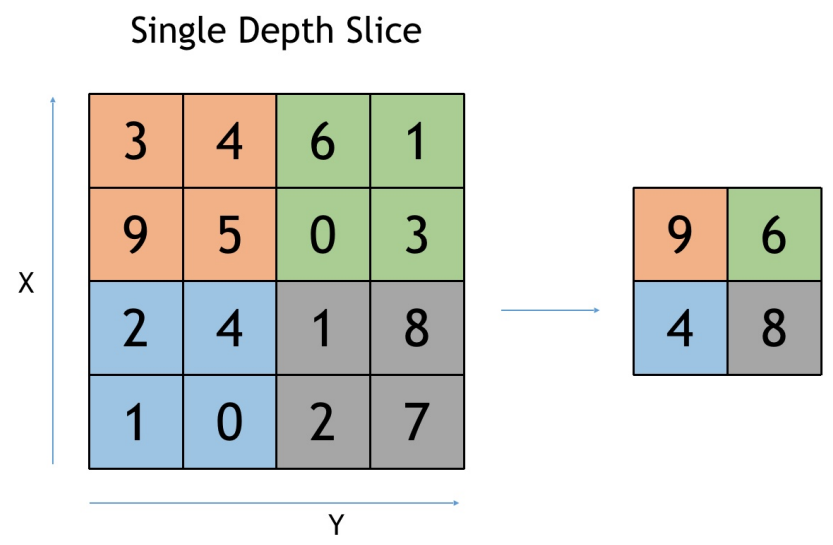

Figure 2.15: Example of max pooling with a $2 \times 2$ filter and stride of 2 .

Apart from max pooling, other pooling functions are possible, e.g., average pooling or $L 2$-norm pooling.

\subsubsection{Overfitting and dropout layer}

Overfitting is the problem characterized by a neural network fitting the noise of the input. At first, the boundaries of classes in the training data will be represented very precisely with an overfitted model. However, when new data arrives, the model will have much lower accuracy in classification, since it fitted all the outliers in the training 
data. Figure 2.16 illustrates the problem of overfitting. In the figure on the left, the curve dividing red points and blue points in the feature space perfectly classifies the points given as training data. However, when new points need to be tested (shown as transparent circles in the figure on the right), the overfitted non-linear classifier will provide worse performance than a linear classifier, which is shown by a dashed line.
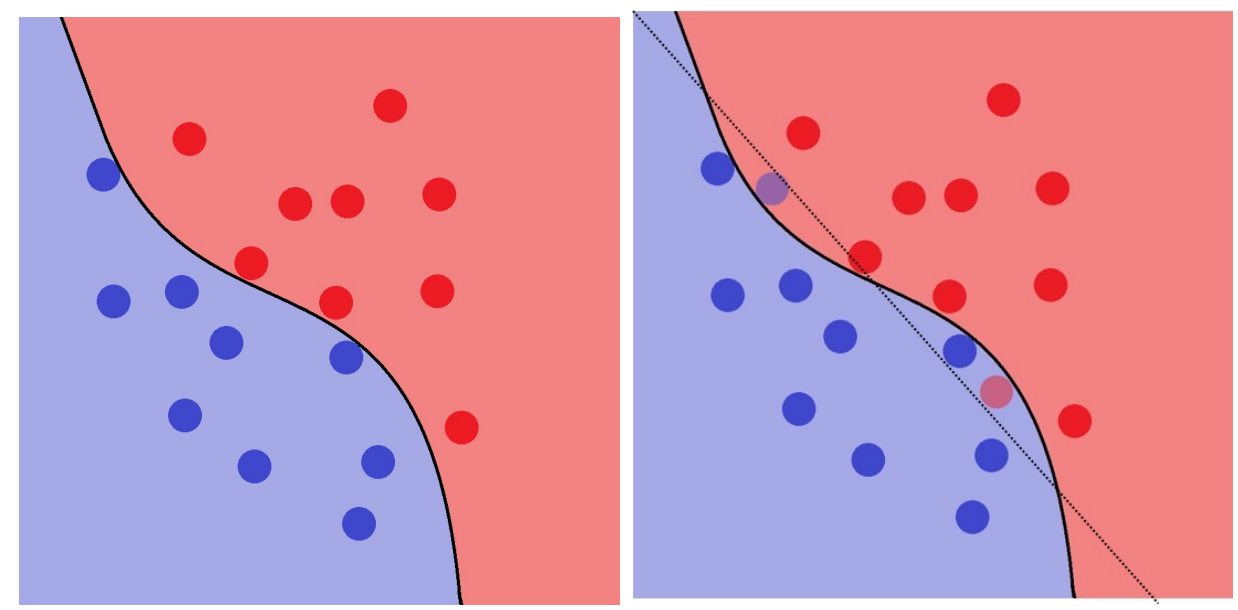

Figure 2.16: An example of overfitting: training data (left), and new test data (right). Please refer to the text for further explanation of the example.

There are basically two types of solutions to avoid overfitting: to increase the amount of training data, or to perform regularization during the learning. In real-life applications, the amount of training data is limited, so the second type of solution is used most frequently.

L2 regularization is the most commonly used type of regularization. If we let the regularization strength be $\lambda$, for every weight $w$, we add $\frac{1}{2} \lambda w^{2}$ to the objective function. In $L 1$ regularization, which is also commonly used, we add $\lambda|w|$ to the objective.

Srivastava et al. [48] proposed dropout regularization, which is widely used when training convolutional neural networks. A hyperparameter used in dropout regularization is the dropout keeping probability $p$. We only keep neuron connections of probability $p$. Figure 2.17 presents an illustration by Srivastava et al. [48] to explain how dropout regularization works. 

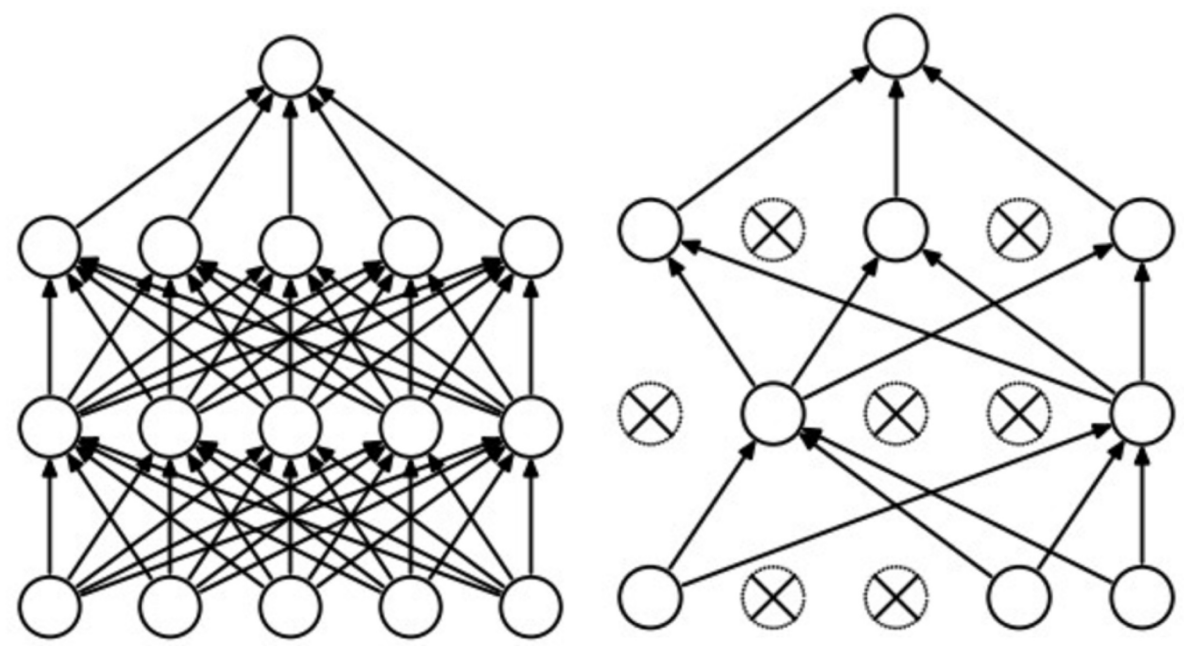

Figure 2.17: A standard neural network (left) and a network with dropout regularization added (right). Only connections with a probability higher than a parameter $p$ are kept in the network. Image courtesy of Srivastava et al. [48].

\subsection{Long Short-Term Memory (LSTM) network}

LSTM is a prominent variation of Recurrent Neural Networks (RNNs). As its name implies, the units in a RNN form a cycle. RNNs not only consider the current input, but also "remember" previous inputs. Figure 2.18 presents an example architecture of a RNN [12].

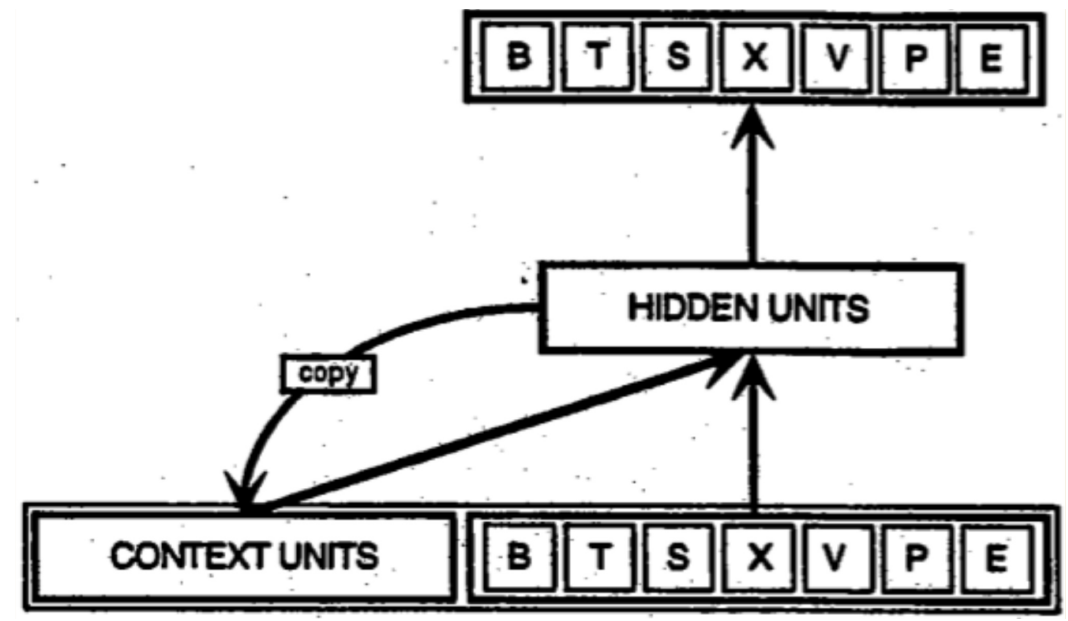

Figure 2.18: Simple recurrent network. Image courtesy of Elman et al. [12].

The context units represent the last output of the RNN. Within the RNN, the 
output computed at time $t-1$ will influence the output at time $t$. Therefore, RNNs are commonly used to process sequential data, and used for applications such as speech recognition. The sequential information is kept in the hidden states of hidden units. If we denote the hidden state of time step $t$ as $h_{t}$, then it can be influenced by $h_{t-1}$ as follows:

$$
h_{t}=\phi\left(W x_{t}+U h_{t-1}\right),
$$

where $U$ is the transition matrix to transit the former hidden state to the current hidden state, and $W$ is a weight matrix. The function $\phi$ is either a logistic sigmoid function or a tanh function.

Instead of training RNNs with backpropagation, we perform backpropagation through time to achieve the backpropagation of errors and perform gradient descent. However, this may still incur in the problem of gradients vanishing. To solve this issue, Sepp et al. [22] propose the long short-term memory network as a solution. Each LSTM cell has three gates. The gates decide when to read, write, and erase information in a unit. These gates manipulate the unit's information with their own weights. Their weights are also updated through backpropagation. Figure 2.19 shows the structure of an LSTM cell [17].

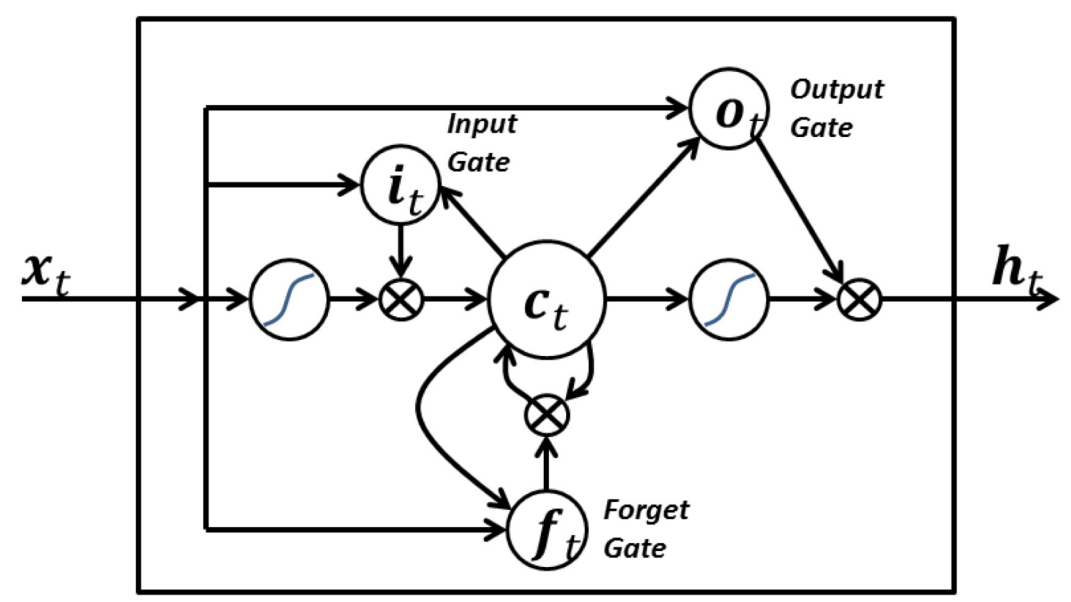

Figure 2.19: A Long Short-Term Memory cell. Image courtesy of Graves et al. [17]. 


\subsection{Object detection and object tracking with neu- ral networks}

Deep neural networks have recently been used for several computer vision tasks [18], including object detection [45], semantic segmentation of images [10,34], generation of descriptions for images [26], and object recognition from multiple views [49].

Similar to the ImageNet Large Scale Visual Recognition Challenge (ILSVRC), we define object detection as the task of localizing all the objects present in an image [45]. The ILSVRC, held since 2010, is a competition ran to determine what are the best state-of-the-art methods for addressing three major tasks: classification, localization, and object detection. In the object detection task, there can be any number of objects in an image, and the measure to evaluate results is the mean Average Precision (mAP).

In 1998, LeCun et al. [31] introduced LeNet, which is the first successful convolutional neural network. It has originally been used to detect and recognize handwritten digits.

Furthermore, Krizhevsky et al. [29] proposed AlexNet, which firstly introduced the idea of stacking convolutional layers together. It obtained the first place in the 2012 ImageNet ILSVRC challenge, outperforming the second place with $10 \%$ less error.

Zeiler and Fergus [59] won the 2013 ILSVRC challenge with ZFNet. Based on AlexNet, ZFNet expands the size of its middle convolutional layer, and reduces the stride and kernel size of its first layer.

GoogLeNet developed by Szegedy et al. [51] is the ILSVRC 2015 winner. This architecture has an inception module that significantly reduces the number of parameters in the network. At the top of this network, an average pooling layer is used instead of a fully-connected layer, also to reduce the number of parameters needed.

VGGNet, introduced by Simonyan et al. [47], is a convolutional neural network architecture that also performed well in 2014. It has 16 convolutional and fullyconnected layers. The size of the convolutional filters and of the pooling filters is uniform across the network, being fixed at $3 \times 3$ filters for convolution and $2 \times 2$ for pooling, in all of the convolutional and pooling layers of the network, respectively. Its performance is determined mainly by the expensive and memory-costly fullyconnected layers.

As mentioned before, GoogLeNet was the winner of the ILSVRC object detection 
in 2015 [45], and introduced an Inception Module in its architecture. The Inception Module is illustrated in Figure 2.20. This was inspired by Lin et al. [37] in their work Network-in-Network. However, in GoogLeNet, $1 \times 1$ convolutional layers are also used to control the size of the network. Arora et al. [9] states that we can optimize the network architecture by analyzing the statistics of the correlation between activations of the last layer, and then cluster together neurons with highly correlated outputs. This assumes that these units clustering in a local region can be covered by $1 \times 1$ convolutions in the next layer, and that there are also more spread-out clusters that can be covered with $3 \times 3$ or $5 \times 5$ convolutions. Szegedy et al. also suggest that pooling can incur in extra benefits to current convolutional networks [51].

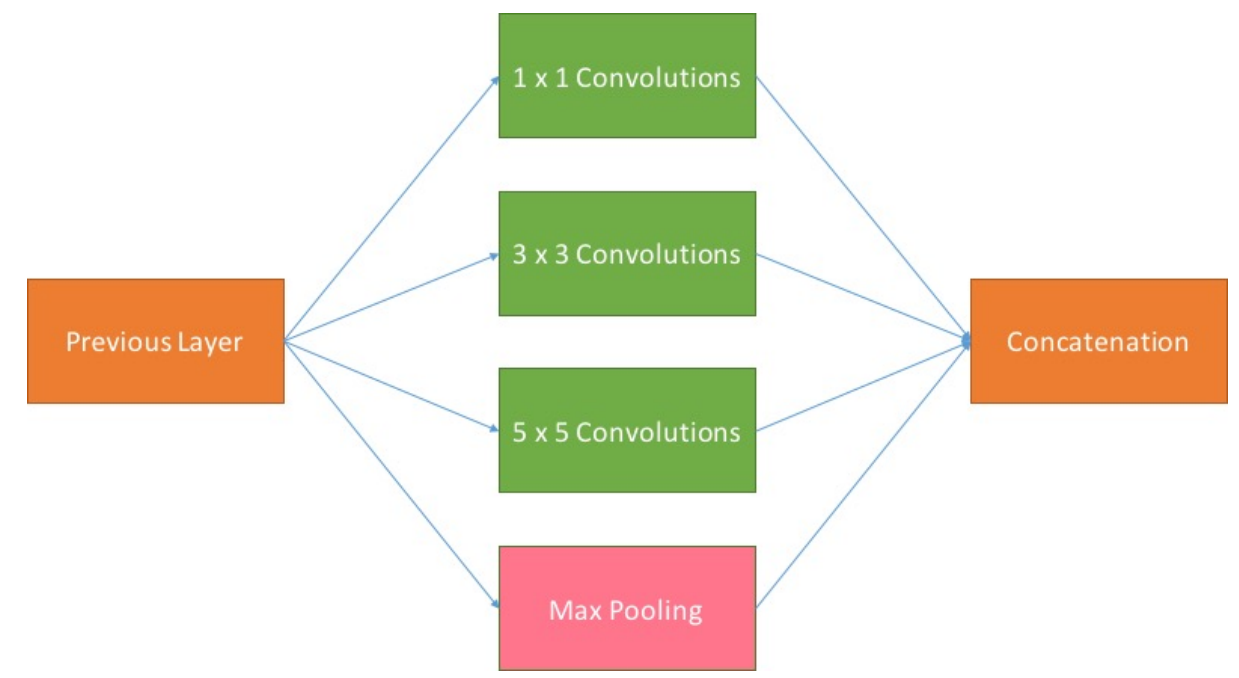

Figure 2.20: Naive Inception Module used in GoogLeNet.

When using $5 \times 5$ convolutions at higher layers in the Naive module, the computation can become quite expensive at deeper layers of the network. To reduce the dimensions of $3 \times 3$ and $5 \times 5$ convolutions, $1 \times 1$ convolutions are used. This is because low dimensional embeddings can still contain a lot of information about a larger area. [51] Embedding here means convolutions with large-sized kernels. Therefore, when used with $3 \times 3$ and $5 \times 5$ convolutions, $1 \times 1$ convolutions are used first. Figure 2.21 is an illustration of an advanced version of the Inception Module.

There are 22 layers with parameters in GoogLeNet model version 1 [51]. It is worth mentioning that the network uses an average pooling layer instead of a fullyconnected layer, based on the suggestion by Arora et al. [9]. They state that this 


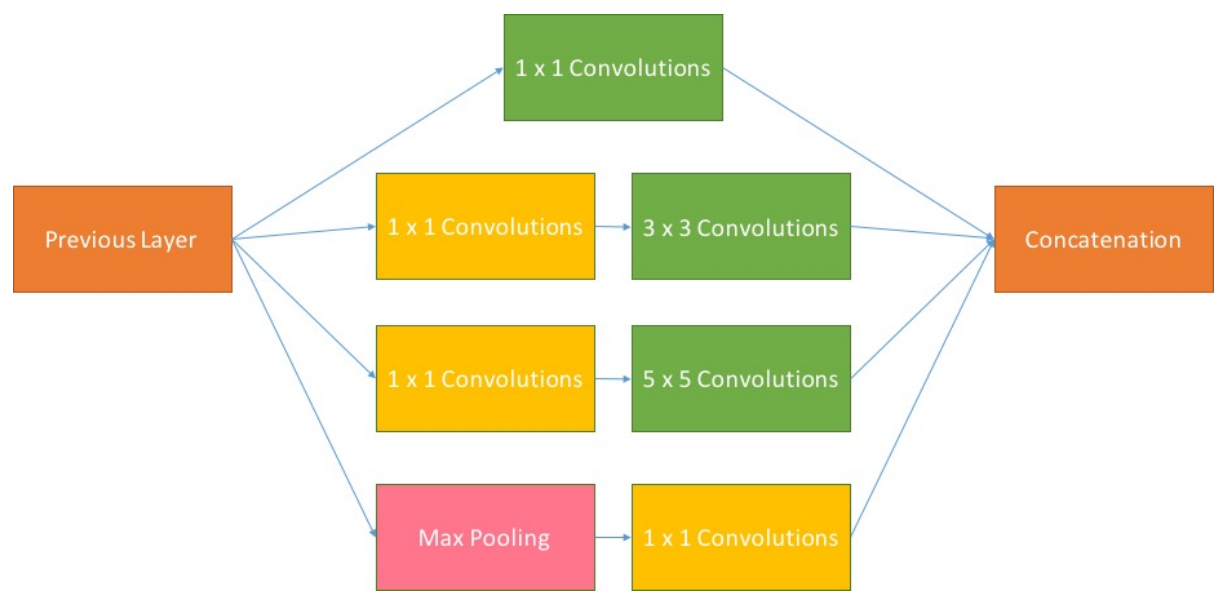

Figure 2.21: Advanced Inception Module used in GoogLeNet.

choice helps to increase the top- 1 accuracy by $0.6 \%$ in ILSVRC. Figure 2.23 provides an illustration of GoogLeNet with all the layers included.

In this thesis, we use GoogLeNet to extract features in images. We chose GoogLeNet as it was ranked first in the ILSVRC object detection competition at the time that this research was initiated [45]. To perform full object detection, GoogLeNet uses an approach similar to R-CNN [16] with the region classifier implemented with the Inception model. The approach uses Selective Search [54] with multi-box predictions [13] to obtain region proposals, and then uses the Inception model to classify the region proposals. Instead of following this original approach, we used an approach similar to Overfeat [46]. The approach used by Overfeat [46] is to train the system to output both classes and the predicted location and size of bounding boxes of objects relative to each cell of the input. More recent approaches that also leverage this idea call the resulting networks region proposal networks [42].

Huval et al. [25] also use the Overfeat approach in their work for implementing a real-time vehicle detection system on highways. They pointed out that, during the merging of bounding boxes, when two bounding boxes overlap, the algorithm may decide that there is a third bounding box in the middle of these two bounding boxes. This is one of the reasons why we introduce an LSTM network as a tracker to correct the network's predictions from frame to frame.

In terms of the state-of-the-art in object detection, currently the best performing method is the BDAT model proposed by Shuai et al. [4]. This model is the winner of 


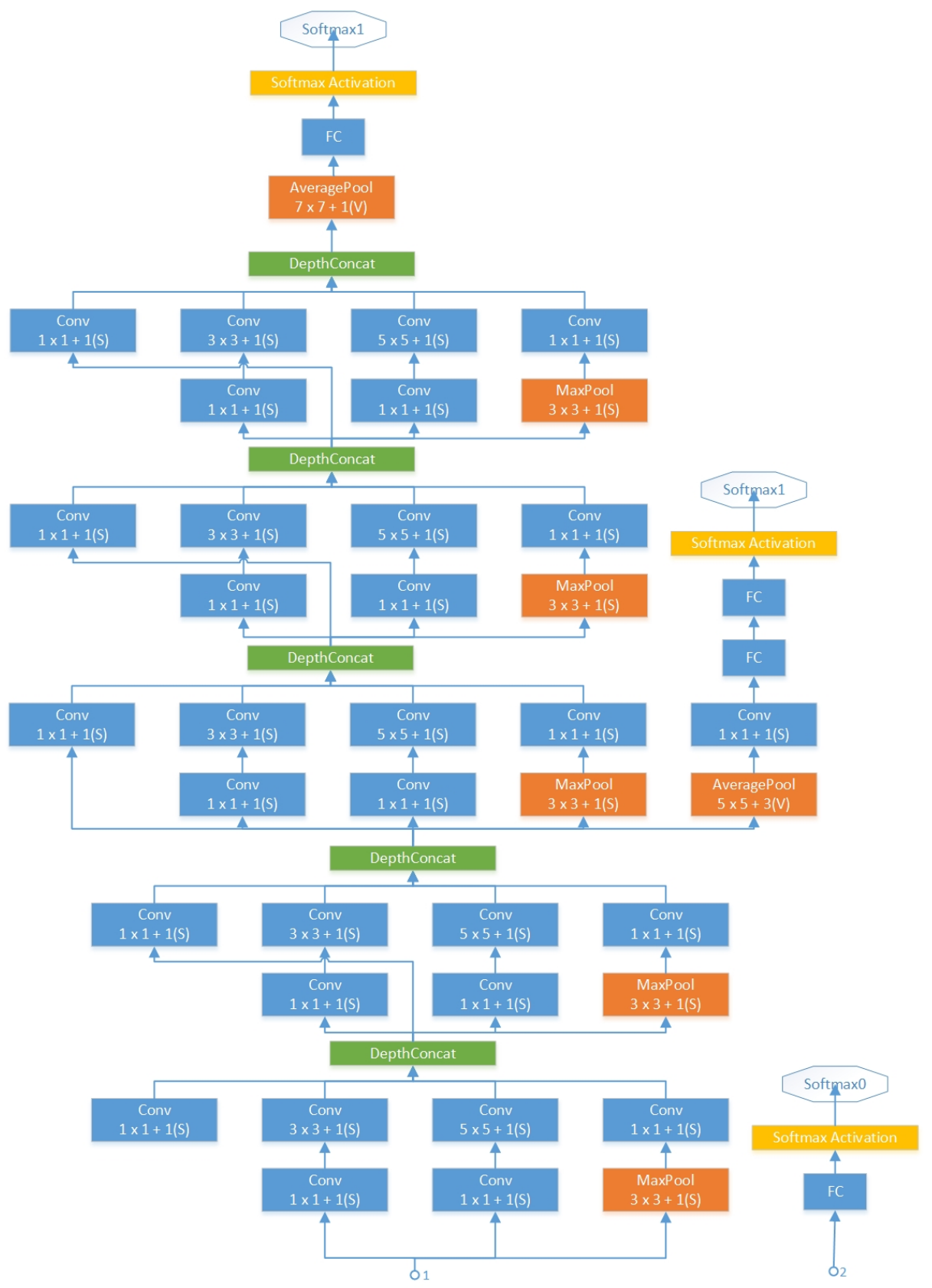

Figure 2.22: Illustration of GoogLeNet with all layers included (continued in the next figure). Image courtesy of Szegedy et al. [51]. 


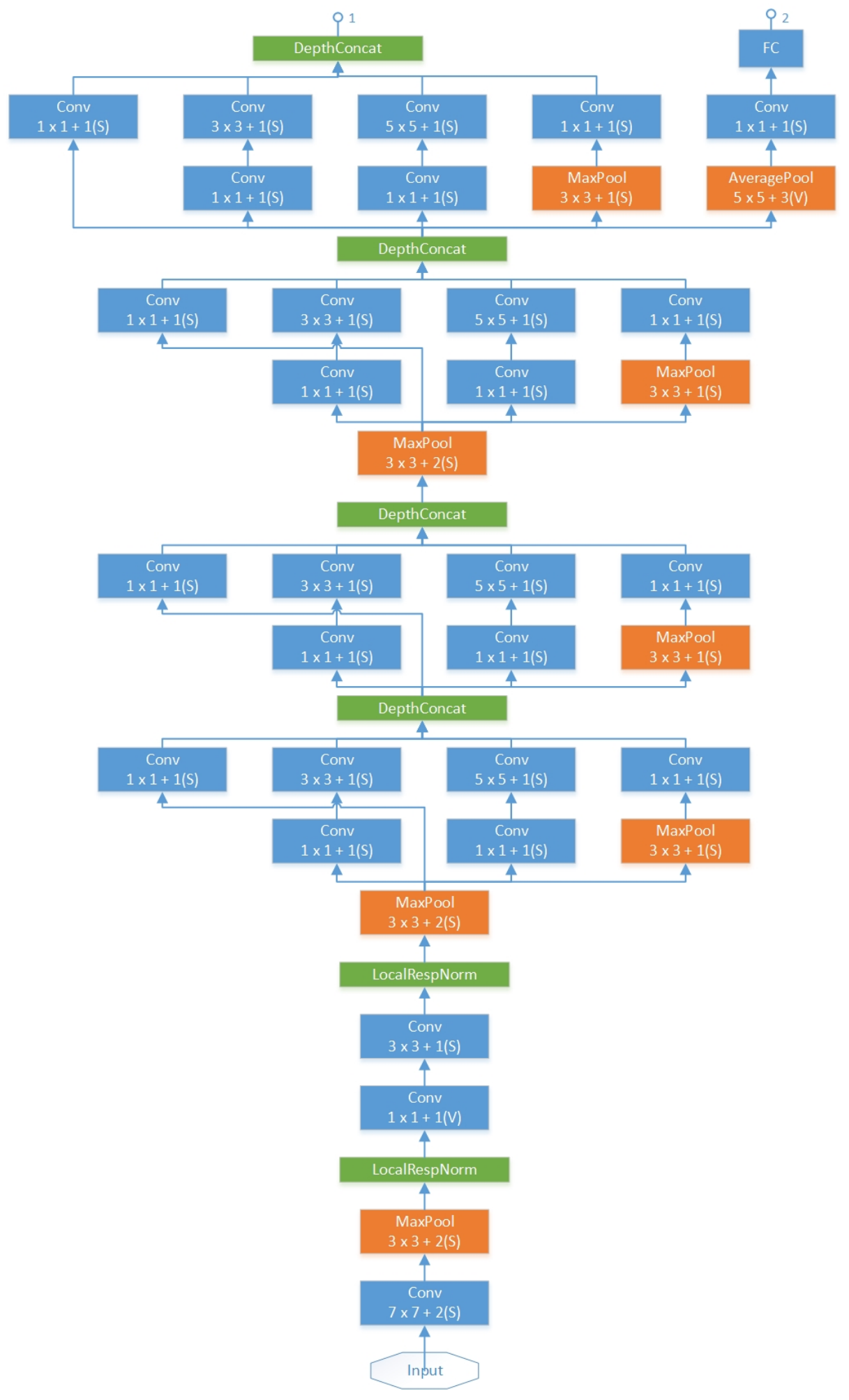

Figure 2.23: Illustration of GoogLeNet with all layers included (continued from the previous figure). Image courtesy of Szegedy et al. [51]. 
the ILSVRC 2017 object detection task, achieving a mean Average Precision of 0.73. However, Shuai et al. have not published their work yet, so we do not know the exact architecture of their model to contrast it to our work. In the report of the ILSVRC 2017 [4], it is mentioned that the BDAT model considers the scale and context of objects, learned with deep convolutional neural networks.

Similar to Ning et al. [40], we combine a ConvNet followed by an LSTM for object detection and tracking. However, Ning et al. use the YOLO approach [41] for object detection, since it provides real-time detection, performing faster than GoogLeNet. In contrast, we argue that in our application which is facilitating the creation of interactive videos, accuracy is more important than speed. A more accurate detection of bounding boxes will lead to a more pleasant experience for the viewer of the video, or reduce the amount of manual work if the video is post-processed by a human.

Moreover, Ning et al. apply the tracker to the bounding boxes predicted by the detector. On the other hand, the input of our LSTM is a concatenated feature vector from the previous frame of the video and a feature vector generated by ConvNet for the current frame. The feature vector contains both the class information and the location information. Thus, giving the feature vector as input to the LSTM provides more information for learning correlations between frames, since class information has a significant influence on localization. The LSTM then outputs an adjusted feature vector. We can then apply a classifier and regressor on the feature vector to obtain the predicted confidences and predicted bounding boxes, respectively.

Moreover, our overall goal is different from Ning et al. Their goal is to achieve real-time tracking of one specific object, while our goal is to obtain more accurate results in the detection of all objects belonging to the target class.

Liang and $\mathrm{Hu}$ [32] also propose the use of recurrent networks for object detection. However, they use the network to mimic recurrent connections that exist in the human visual system, and apply it mainly to static images. Our goal is different as we use a recurrent network (LSTM) to track an object across multiple frames.

In terms of the state-of-the-art in object tracking, an example approach is the LSART method proposed by Sun et al. [50]. This method is the winner of the VOT 2017 challenge [6], and achieves an average bounding box overlap of 0.323. The expected average overlap used in the VOT challenge measures the expected no-reset overlap of a tracker run on a short-term sequence [6]. LSART combines kernelized ridge regression (KRR) with a convolutional neural network (CNN). They use the 
KRR model for holistic tracking, while the CNN model is used for tracking local regions.

\subsection{Implementation tools}

In the follow sections, we provide some discussion on the tools that we use to implement our object detection method.

\subsubsection{Tenforflow}

Tensorflow is a library for machine learning algorithms [7]. It is Googles secondgeneration system for large-scale machine learning models [7]. In Tensorflow, a computation model can be described by a directed graph, which represents a dataflow computation. An example of Tensorflow graph is given in Figure 2.24 [7]. Each graph is composed of a set of nodes, and each node has zero or more inputs, as well as zero or more outputs. A node is an instantiation of an operation, and an operation represents an abstract computation. While an operation can have several attributes, all attributes must be predefined at graph construction. The types of operations are listed in Figure 2.25 [7].

\subsubsection{TF-Slim}

TF-Slim is a lightweight library for defining, training and evaluating complex models in Tensorflow. Components of TF-slim can be freely mixed with native Tensorflow, as well as other frameworks [53]. It simplifies operations with neural networks, and helps to reduce boilerplate code when defining models through the use of argument scoping and high-level layers and variables. It also helps to simplify the process of building models through regularizers, and provides some well-known models of neural networks, e.g., VGG, AlexNet. Apart from these, it is easy with TF-slim to extend models through checkpoints. There are several components available in TF-slim, as listed in Table 2.1. 

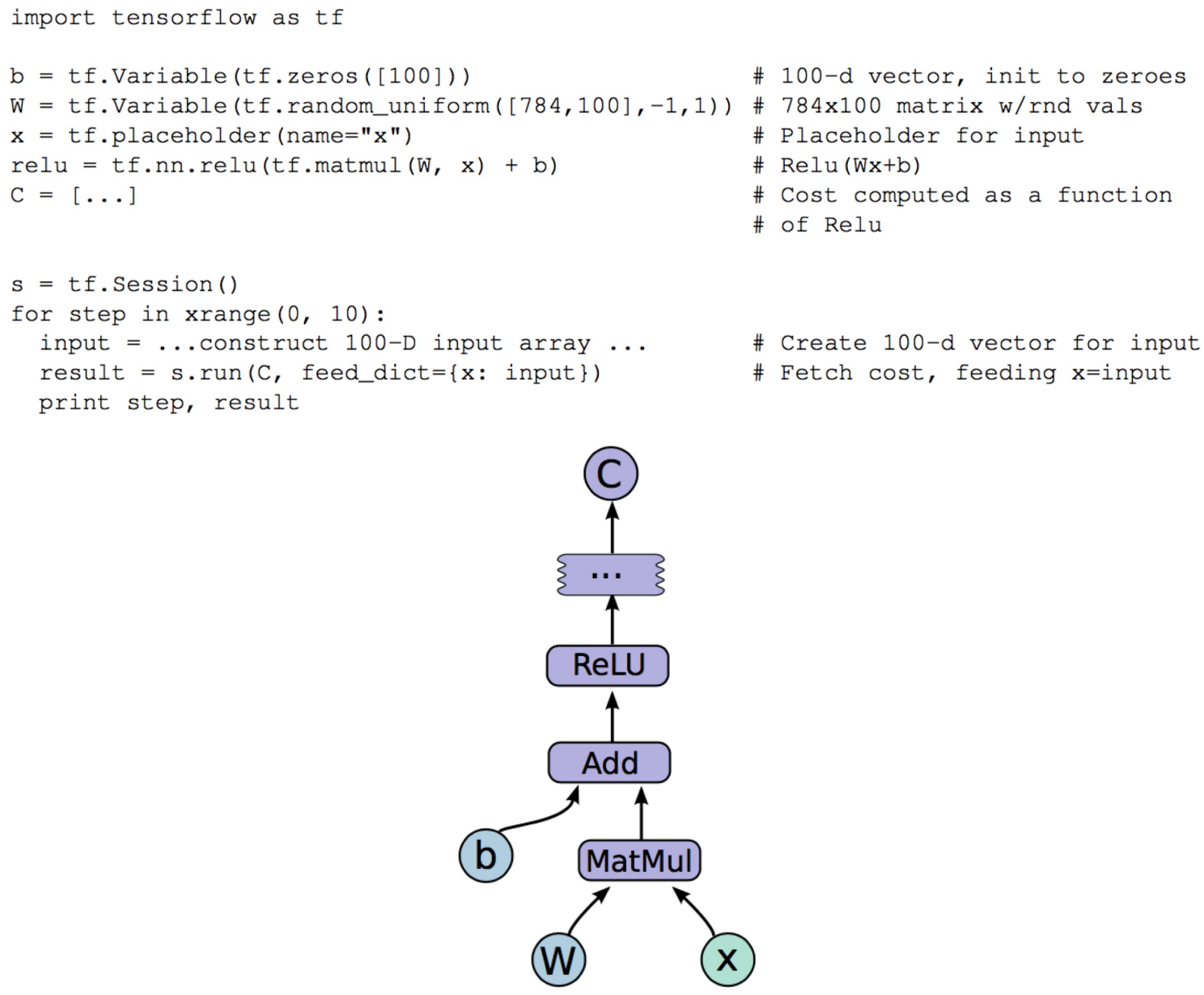

Figure 2.24: Example Python code (above) and its corresponding computation graph (below). Image courtesy of Abadi et al. [7].

\begin{tabular}{|l|l|}
\hline Category & Examples \\
\hline Element-wise mathematical operations & Add, Sub, Mul, Div, Exp, Log, Greater, Less, Equal, ... \\
Array operations & Concat, Slice, Split, Constant, Rank, Shape, Shuffle, ... \\
Matrix operations & MatMul, MatrixInverse, MatrixDeterminant, ... \\
Stateful operations & Variable, Assign, AssignAdd, ... \\
Neural-net building blocks & SoftMax, Sigmoid, ReLU, Convolution2D, MaxPool, ... \\
Checkpointing operations & Save, Restore \\
Queue and synchronization operations & Enqueue, Dequeue, MutexAcquire, MutexRelease, ... \\
Control flow operations & Merge, Switch, Enter, Leave, NextIteration \\
\hline
\end{tabular}

Figure 2.25: Types of Operations Available in Tensorflow. Image courtesy of Abadi et al. [7]. 


\begin{tabular}{|l|l|}
\hline Component & Usage \\
\hline Arg_scope & $\begin{array}{l}\text { provides a new scope named arg_scope that allows } \\
\text { a user to define default arguments for specific } \\
\text { operations within that scope. }\end{array}$ \\
\hline Data & $\begin{array}{l}\text { contains TF-slim's dataset definition, data providers, } \\
\text { parallel_reader, and decoding utilities. }\end{array}$ \\
\hline Evaluation & contains routines for evaluating models. \\
\hline Layers & $\begin{array}{l}\text { contains high level layers for building models using } \\
\text { Tensorflow. }\end{array}$ \\
\hline Learning & contains routines for training models. \\
\hline Losses & contains commonly used loss functions. \\
\hline Metrics & contains popular evaluation metrics. \\
\hline Nets & $\begin{array}{l}\text { contains popular network definitions such as VGG } \\
\text { and AlexNet models. }\end{array}$ \\
\hline Queues & $\begin{array}{l}\text { provides a context manager for easily and safely } \\
\text { starting and closing QueueRunners. }\end{array}$ \\
\hline Regularizers & contains weight regularizers. \\
\hline Variables & and manipulation. \\
\hline
\end{tabular}

Table 2.1: Main Components available in TF-Slim

\subsubsection{Optimizers used for learning}

There are seven types of optimizers in tensorflow. However, only four of the optimizers are commonly used with neural networks: Gradient Descent Optimizer, Momentum Optimizer, RMS Prop Optimizer, and Adam Optimizer. The major difference between the optimizers is how they update the parameters of the networks.

In the SGD Optimizer, a parameter $x$ is updated by its gradient $d x$ according to:

$$
x=x-r d x
$$

wherer is the learning rate, which is a hyperparameter. 
In the Momentum Optimizer, the change of parameters is analogized to a rock rolling down a landscape, according to a parameter $v$ which stands for the integrated velocity. The parameter $x$ is updated according to:

$$
\begin{gathered}
v=m v-r d x, \\
\text { with } x=x+v,
\end{gathered}
$$

where $m$ is the momentum. It is a hyperparameter defined by the user.

In the RMS Prop Optimizer [21], the learning rate is attenuated to reduce its impact on the parameters. The parameter $x$ is updated according to:

$$
\begin{aligned}
& c=\lambda c+(1-\lambda) d x^{2}, \\
& \text { with } x=x-r \frac{d x}{c+\epsilon},
\end{aligned}
$$

where $\epsilon$ determines the size of the error for termination, $\lambda$ is the decay rate, $r$ is the learning rate, and $c$ is referred to as the "cache". $\epsilon, r$, and $\lambda$ are hyperparameters.

The Adam Optimizer [27] combines the ideas of both the Momentum Optimizer and the RMS Prop Optimizer. The parameter $x$ is updated according to:

$$
\begin{gathered}
m=h_{1} m+\left(1-h_{1}\right) d x, \\
\text { with } v=h_{2} v+\left(1-h_{2}\right) d x^{2}, \\
\text { and } x=x-r \frac{m}{v+\epsilon},
\end{gathered}
$$

where $h_{1}, h_{2}$ and $\epsilon$ are hyperparameters. 


\section{Chapter 3}

\section{Object detection and tracking}

The major task that we address in this thesis is object detection in videos. Object detection, as its name implies, consists in detecting and localizing objects in an image. This is typically accomplished by extracting features from the image content and determining the location and class of an object based on these features. In the case of videos, spatio-temporal features can provide additional information for detecting and tracking objects in multiple frame sequences. In our work, we use neural networks to extract both types of features, where the neural networks are learned from training data in the form of single images and videos. In the next sections, we introduce our object detection method in more detail.

\subsection{Overview}

There are three major components that compose our object detection method: a convolutional network (ConvNet) that acts as the feature extractor for single frames, an LSTM network acting as a tracker that incorporates spatio-temporal processing, and two fully-connected layers acting as classifier and regressor.

We use GoogLeNet [51] as the convolutional network that extracts image features for classification. However, unlike the original GoogLeNet, we feed its output to selected layers of the Overfeat [46] network, so that we can simultaneously perform classification and localization, simplifying the structure of the network. The original Overfeat [46] used a 5-layer convolutional network similar to AlexNet [29] for the initial processing of the input image. We chose to exchange this component for GoogLeNet since this network is deeper than AlexNet and had the best performance in the Imagenet Large Scale Visual Recognition Challenge (ILSVRC) in 2015 [45]. 
Before feeding the output of GoogLeNet to the classifier and regressor, we process its output with an LSTM network that acts as a tracker and adjusts the features extracted by GoogLeNet to account for temporal coherence. Since typically there are strong temporal relations between frames of video sequences, we use the LSTM network to learn how spatial features evolve in time.

As discussed in Chapter 2, Ning et al. [40] also propose to combine convolutional networks with LSTMs for tracking. However, the LSTM learns to track the detected bounding boxes, represented as 2D coordinates and box dimensions. In our work, we use the LSTM to adjust the features output by the ConvNet, which are then forwarded to a classifier and regressor layer for predicting the objects' bounding boxes and confidence of detection. In addition, we do not require an extra loss function for training the LSTM network, since both the ConvNet and LSTM use the same set of loss functions. One disadvantage of our solution is that training the LSTM to adjust the features provided by the convolution in a meaningful manner requires considerable more computation. However, we show experimentally that this leads to advantages in terms of higher accuracy in object detection.

In the next sections, we first explain the architecture of our full network in detail, and then discuss the preparation of data and training of the network.

\subsection{Architecture of the network}

As illustrated in Figure 3.1, the input of the ConvNet portion of our network is an image of dimensions $640 \times 480$ with 3 color channels. The network convolves the image with several layers to extract image features. The specific convolutions performed by the network are summarized in Figure 3.1. The output of the network is then a feature vector of dimensions $20 \times 15 \times$ (\#classes), where \#classes is the number of object classes handled by the network. Then reason for this dimensionality is that, similar to the work of Sermanet et al. [46], the input image is divided into cells of size $32 \times 32$ pixels, and the detection provides a feature vector for each cell and class, which can be used to derive the confidence that an object of a given class is present in a given cell.

We then feed the output of the ConvNet for two consecutive frames into the LSTM, as illustrated in Figure 3.2. There are \#classes steps in this LSTM network, each batch is 300 , and the output length is 1 . The LSTM cell will run \#classes 
times, and there will be 300 inputs of length 2 as the input tensor. An LSTM cell has three gates that control the input of the cell, the output of the cell, and whether the cell should forget the data that it retains. These three gates are represented by three parameters, which are learned during the training process. The input goes through an LSTM cell to provide an activation. The activation is then fed back to the cell itself as the input for the next step. This feedback process is repeated \#classes times. We concatenate all the outputs of the LSTM to get a one-dimensional tensor, whose length is $300 \times$ (\#classes). This is the final feature vector for the current frame, incorporating information extracted from the two consecutive frames in the video. We provide this feature vector as input to the classifier and regressor, to obtain the predicted confidences and bounding boxes.

The classifier consists of two layers: a fully-connected layer to obtain logits from the features; and, a softmax layer, which normalizes logits into confidences. The softmax layer computes the function:

$$
\sigma\left(z_{j}\right)=\frac{e^{z_{j}}}{\sum_{k=1}^{K} e^{z_{k}}},
$$

where $z_{j}$ is the logit for class $j$, and $K$ is the total number of classes. The function normalizes real-valued class scores to values between 0 and 1 . In addition, the sum of scores for all classes is 1, providing an estimated probability distribution. The probabilities represent the confidence that an object of a given class is present in each cell of the input.

Moreover, the goal of the regressor is to predict four bounding box parameters from the input features: $o x$, the distance in the horizontal direction from the center of the cell to the center of the bounding box, oy, the vertical distance from the center of the cell to the center of the bounding box, and the width and height of the bounding box in the cell. With these four values, a more accurate bounding box can be reconstructed from a prediction performed on a $15 \times 20$ grid. As discussed by Sermanet et al. [46], these values can be used to predict the size of the object in the current cell, which is critical for prediction in situations where the detected objects vary significantly in their size, influencing the accuracy of the detection. Moreover, when a cell includes a part of the target object, if this part is highly identifiable but not close to the center of the object, the localization will be influenced. Thus, predicting the relative position to the current cell addresses this issue. 
The regressor also consists of two fully-connected layers. Our regressor is the same as in the Overfeat [46], however, we changed the spatial size from $1 \times 1$ to $15 \times 20$. The first layer is used to obtain a tensor of shape $15 \times 20 \times(16 \times($ \#classes $))$ from the features. The next layer produces a tensor of shape $15 \times 20 \times(4 \times(\#$ classes $))$, which represents the predicted four bounding box parameters for each cell.

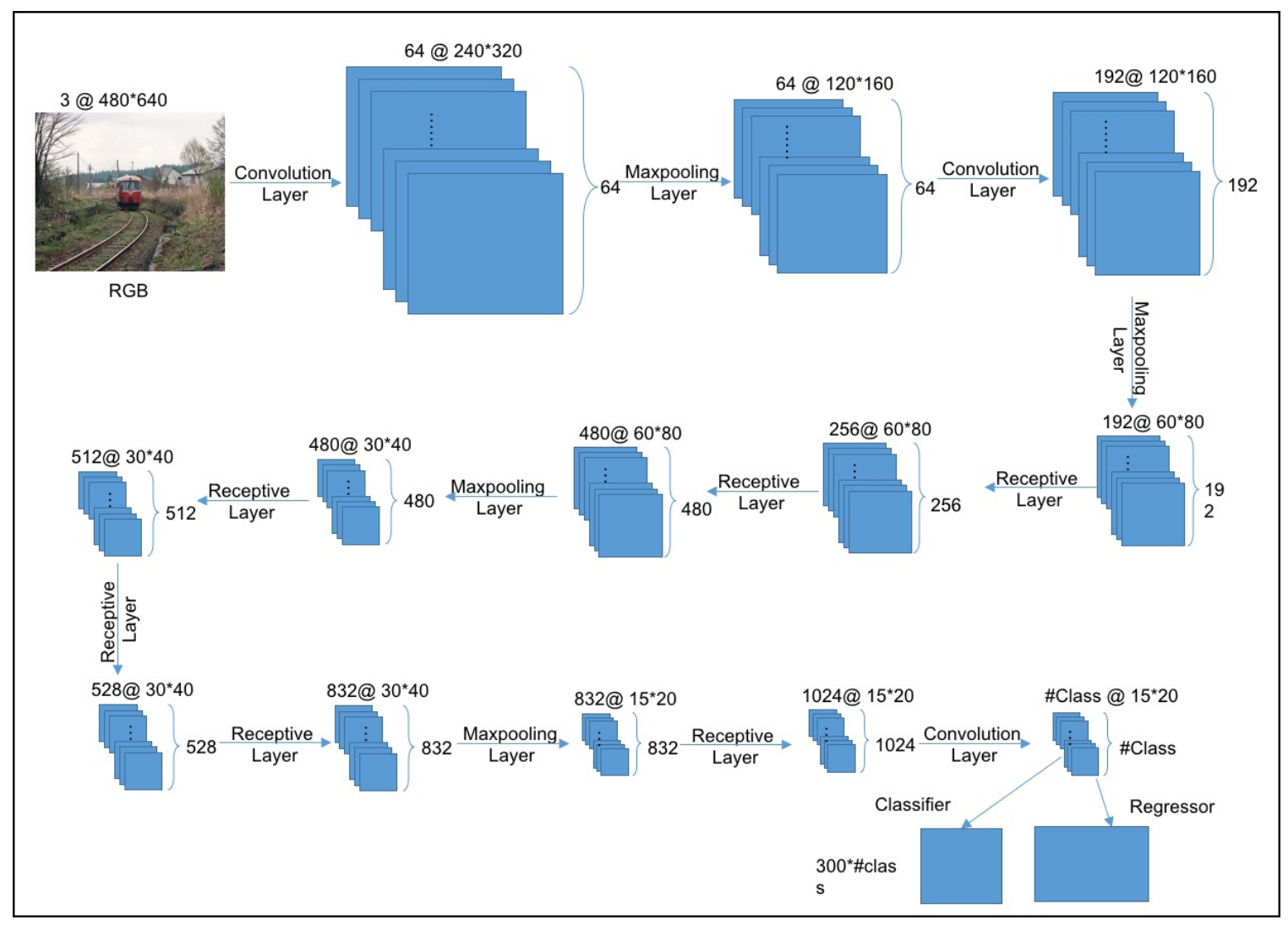

Figure 3.1: The architecture of the ConvNet portion of our network. For each layer, we denote the type of unit, the number of units in the layer, the size of the image block processed by each unit, and the size of the output.

In summary, we use a ConvNet based on GoogLeNet to produce intermediate representations of images. Then, we provide these intermediate representations to the LSTM network for tracking. In a video, there are correlations between the objects detected in the previous frame and the objects detected in the current frame. We use the LSTM network to learn these correlations and use these correlations to adjust the predictions that have been made by the ConvNet. Finally, we provide the output of the LSTM to a classifier and regressor to obtain the final results of predicted 


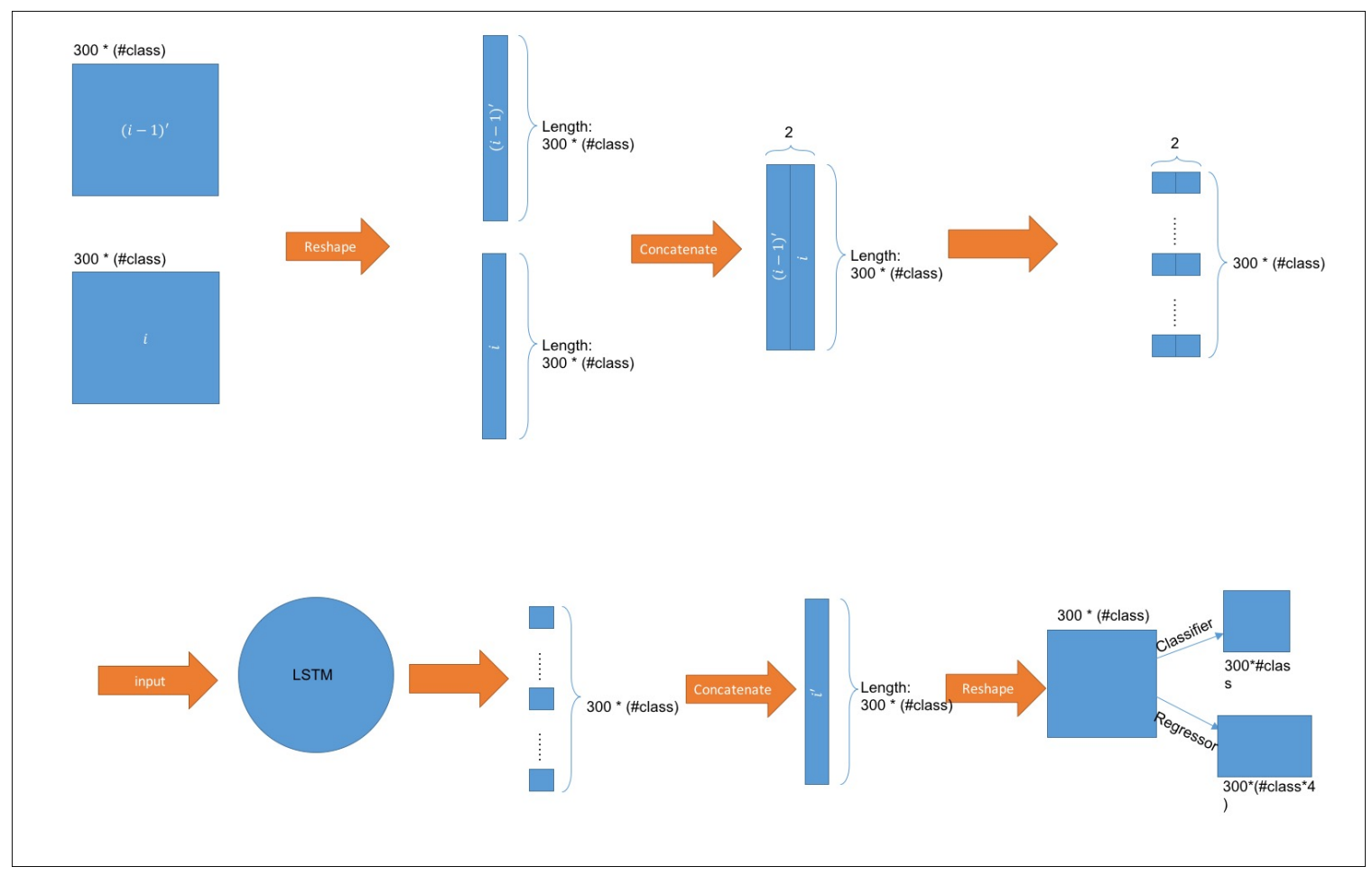

Figure 3.2: The architecture of the LSTM in our network. We denote the operations that are applied to the data before and after feeding it to the LSTM cells. 
confidences and bounding boxes.

\subsection{Pre-processing}

Before training the network, we pre-process the input data to transform it into a consistent format. We first resize all of the input images and video frames to a standard size of $640 \times 480$ with 3 RGB color channels, while also rescaling any annotated ground-truth boxes accordingly.

Next, we prepare the ground-truth information for the training step. We divide each image into cells of size $32 \times 32$ pixels, resulting in a grid of $20 \times 15$ cells. For each cell, we verify if the cell intersects any ground-truth bounding boxes in the image. If a cell intersects a bounding box, we record the class of the object and calculate the relative position of the bounding box to the cell.

Relative $x$ coordinate $=x$ coordinate of the center of bounding box $-x$ coordinate of the center of cell Relative $y$ coordinate $=y$ coordinate of the center of bounding box $-y$ coordinate of the center of cell

Figure 3.3: The Relative Position Formulas

We also record the width and height of the bounding box in the cell.

To deal with situations where two objects of different classes appear in the same cell, we compute the L2 distance between the center of the object and the center of the cell, and assign only the object with the shortest distance to the cell. This is reasonable if we assume that the input videos do not depict crowded scenes with several intersecting objects.

Thus, after pre-processing, each image of size $640 \times 480 \times 3$ is associated to a vector of $20 \times 15 \times 1 \times$ (\#classes) flags marking whether an object appears in a cell or not, and a vector of $20 \times 15 \times 4 \times$ (\#classes) bounding box parameters.

\subsection{Training of the neural network}

A workflow of the training of the object detection network is illustrated in Figure 3.4. The images resized by the pre-processing are taken by the neural networks as inputs to predict bounding boxes and confidences for objects. Then, the predicted boxes and confidences, with the ground truth boxes and confidences, are considered by the loss 
function to provide two loss values: one for boxes and one for confidences. These two loss values are considered by the optimizer to train the networks so that the losses are minimized and predictions are improved.

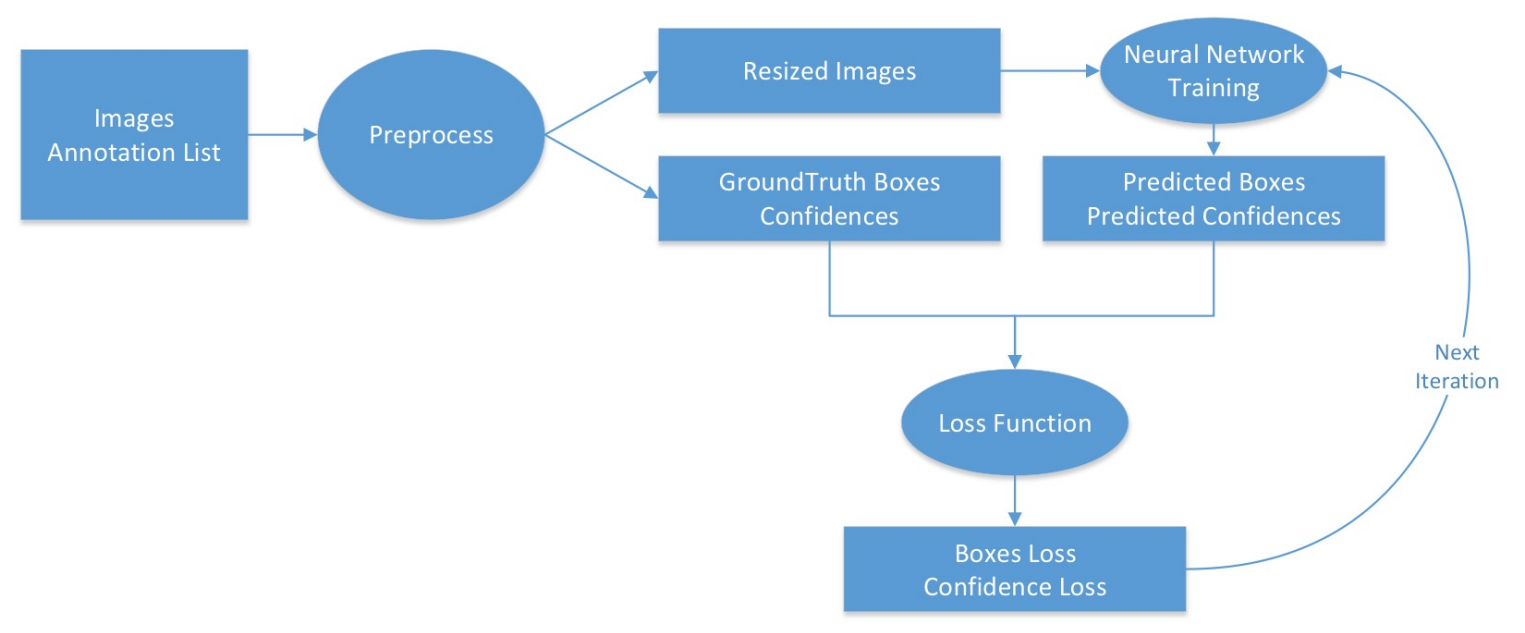

Figure 3.4: Training of the object detection neural network.

Given that training the entire network is a complex and time-consuming task, we break the training into three parts. We start by first training the ConvNet portion of our network. Since the ConvNet is used for feature extraction, we train it with significantly more data, using images from two visual recognition benchmarks: ImageNet ILSVRC [3] and Pascal VOC [14]. There are 1,000 classes of objects in the ILSVRC, and 20 classes of objects in the Pascal VOC. Training the network with these benchmarks is an adequate solution for pre-training the network, since the types of features learned from these datasets are applicable to both single images and videos. More specifically, GoogLeNet was constructed and trained by Szegedy et al. [51] for ILSVRC 2015. Thus, we start with the weights of the network pre-trained for ILSVRC 2014, and further train the ConvNet, classifier, and regressor with Pascal VOC data.

Moreover, the purpose of the LSTM is to learn features in the temporal domain. Thus, we further train it with video data. We use one main resource, the OTB100 dataset [5], along with a few video sequences captured on our own to record specific classes not present in the dataset. The OTB100 dataset contains 100 video clips for visual tracking, and 30 of them are classes that are also included by Pascal VOC [14], which we can potentially use for training our network. In this step of the training, we 
initialize the weights of the LSTM network with values from a random distribution, and train the ConvNet, LSTM, classifier, and regressor together.

The outputs of both the ConvNet and LSTM are of the same dimensions and have the same meaning. Thus, we define a set of loss functions for predicted confidences and predicted bounding boxes, and apply them for training both the ConvNet and LSTM. The loss functions will be further described in Section 3.5.

Furthermore, we use the RMSProp optimizer for the training [21], with standard parameters such as a (slow) learning rate $l r=0.001$, decay rate $d r=0.9$, and $\epsilon=0.00001$ to ensure that the network does not get stuck in a local minimum.

The RMSProp optimizer is discussed in Chapter 2, but we recall here that the parameter $\mathrm{x}$ is updated according to Equation (3.2) and Equation (3.3):

$$
\begin{gathered}
c=\lambda c+(1-\lambda) d x^{2}, \\
x=x-r \frac{d x}{c+\epsilon},
\end{gathered}
$$

where $x$ is the parameter that we are training, $d x$ its derivative, $\lambda$ is the decay rate, $r$ is the learning rate, and $\epsilon$ is the size of the error for termination.

We chose this optimizer because it is more efficient than non-adaptive learning-rate methods such as Stochastic Gradient Descent. In the work of Ruder et al. [44], several optimizers were analyzed, and RMSProp was found to be one of the best performing optimizers, being also used in other Google projects such as AlphaGo [56].

\subsection{Loss function}

We define two loss functions that are used during training: a loss function for predicted confidences and one for predicted bounding boxes.

For each class, to calculate the confidence loss, we use a sparse softmax cross entropy with logits. This enables us to calculate the sparse softmax cross entropy between predicted logits and true confidences. Then, we add together the losses for all the cells, and divide by 300 cells to get a normalized confidence loss. The cross entropy loss $H$ is calculated with:

$$
H(p)=-\sum_{i=1}^{n} p_{i} \log \left(t_{i}\right),
$$


where $p_{i}$ is the class probability according to the logit of the $i$-th cell, $t_{i}$ is the true label of the cell (which assumes a value of 1 if the cell belongs to the current class, or 0 otherwise), and $n=300$ is the number of cells.

We chose the softmax cross entropy function with logits because for the current class, the cell can either belong to this class, or not belong to this class. Moreover, we used the sparse softmax cross entropy with logits instead of the original softmax cross entropy with logits function, because the probabilities of classes are also exclusive. Both of the original function and the sparse function are defined in the API of Tensorflow [7].

For each class, to calculate the boxes loss, we needed to take the classification into consideration. Thus, we multiply the predicted boxes with a mask, which marks if the classification is correct. The loss function $L$ is given by:

$$
\begin{gathered}
r=t-p \otimes l, \\
L=\frac{\sum_{i}^{m}\left|r_{i}\right|}{n},
\end{gathered}
$$

where $t$ is a vector with the ground-truth box parameters of the current class, $p$ are the predicted box parameters of the current class, $l$ is a boolean-value tensor used to ignore the values of predicted boxes that were wrongly classified, $\otimes$ denotes entry-wise multiplication, $m=300 \times 4$ is the total number of box parameters, corresponding to the number of values in the residual $r, r_{i}$ is the $i$-th value of the residual, and $n=300$ is the number of cells.

The total loss used for the training is given by the sum of the confidence loss and the boxes loss. We compute this measure for all the classes.

As we mentioned in Section 3.1, our LSTM network provides as output feature vectors and not predicted bounding boxes such as the work of Ning et al. [40]. The approach of Ning et al. uses the Mean Squared Error as the loss function during training, which is more meaningful in their setting since the output of the LSTM is the final output of the method. In our case, the softmax cross entropy is more meaningful as we calculate the loss after the classifier and regressor layers are applied to the output of the LSTM. In addition, we calculate the loss after the ConvNet is applied to single images, for training only the ConvNet, and after the entire network is applied, when training the full network with video data. 


\subsection{Prediction}

Once the three components of the network are trained, we can apply the entire network to pairs of subsequent frames in a video, and extract object bounding boxes and confidences. For efficiency, we run the ConvNet only once for each frame, and then provide pairs of features extracted by the ConvNet as input to the remaining portions of the network.

\subsection{Implementation notes}

We use Tensorflow [7] for implementing our method. Tensorflow facilitates the construction and training of the network, since we only need to provide the topology of the network and define the loss functions and parameters for the optimizer. Tensorflow then executes the minimize function of RMSProp optimizer which trains the network according to the provided data. 


\section{Chapter 4}

\section{Results}

We first review the datasets that we use in our evaluation. We follow with a discussion of the results obtained for object detection in videos and images.

\subsection{Datasets}

We used four different resources of data to evaluate our system. Two of these resources are datasets of videos. First, we created a mock soda commercial to evaluate our system, where the objects that need to be tracked are soda cans. We created this video as an example of how an interactive video would look like. We also evaluate our system with the OTB100 benchmark [5], which provides 100 video clips for object tracking. Using this dataset also allows us to compare our work to the method of Ning et al. [40].

Although our system is designed to work with videos, we would also like to evaluate its performance on single images, to obtain an indication of its general object detection performance. We use two datasets of images to demonstrate our performance in object detection. We used the Pascal VOC and ILSVRC datasets, since they are the most well-known image datasets, as well as two milestones in object detection. In this context, we compare our work with other neural networks with state of the art performance.

\subsection{Results with video data}

In this section, we evaluate our system with the two resources of video data. All the evaluations were run on an HP desktop with CPU Intel Core $i 7-7700 @ 3.6 G H z \times 8$, 
GPU GeForce GTX 1050. The desktop has python version 2.7.13, Tensorflow version 1.2.0, CUDA 8.0, and CUDNN 5.1 installed. The processing speed we obtained for object detection after training is 6 frames per second.

\subsubsection{Results with our own data}

For our first evaluation, we perform a preliminary test where we train our network with one video and use it to predict objects in a second video. We trained our network with a simple video of soda cans, which has 1,269 frames. The video we recorded for evaluation is more complicated. There are illumination variations, scale variations, and occlusions in the video, which is composed of 487 frames. The illumination variation happens because the light is not always right on top of the objects. Therefore, when we circled around the objects for shooting, the illumination was not consistent. Moreover, the scale variations happen since the objects' sizes are not consistent in the video, since the camera comes near and far the objects. The occlusions can be seen when the soda cans are occluded by other objects or by each other in some frames.

With the experiment, we observed that the accuracy of object detection in the evaluation video reaches $100 \%$ after 10,000 iterations of training. That means that after 10,000 iterations, all detections have Intersection over Union value of over $50 \%$.

To better visualize our work, we choose 7 frames from the video, and compare the predicted bounding boxes with our hand-labeled bounding boxes in Figures 4.1-4.7. In the predicted results, the green bounding box denotes the box with the highest confidence, while the red boxes are all the boxes with confidence higher than 0.5. With this visual inspection, we notice that the results are very close to the ground truth boxes.

Frame 1 and Frame 2 show how our network deals with scalar variations. Frame 1 and Frame 2 are almost from the same angle, but the objects of Frame 2 are $70 \%$ the size of objects in Frame 1. Frame 3 and Frame 4 show how our network deals with occlusion by other objects. The second coke can in Frame 3 is occluded by a bottle, and the invisible part is very limited. Frame 4 especially shows an extreme case of 

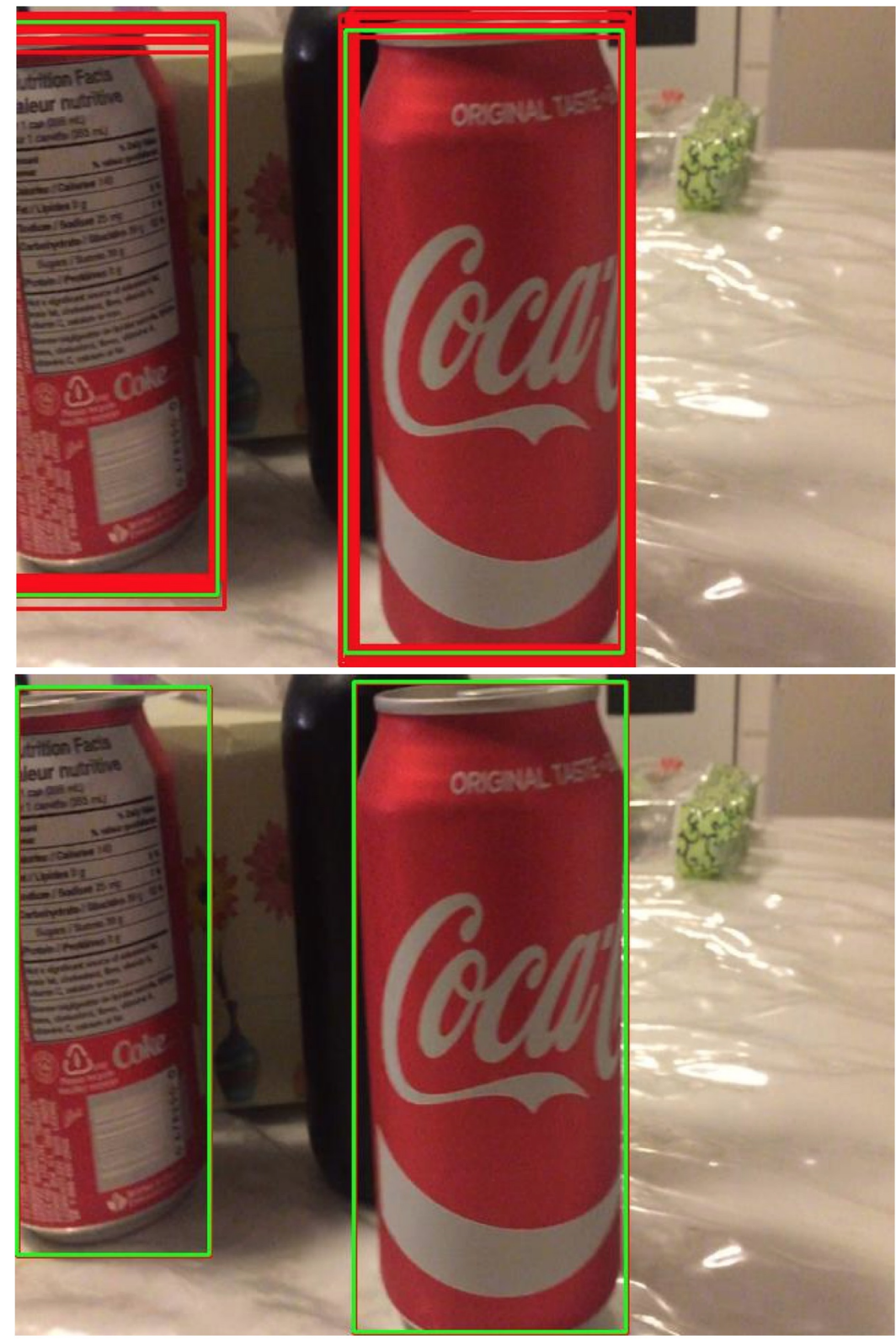

Figure 4.1: Frame 1 with predicted boxes (above) and hand-labeled boxes (below): 


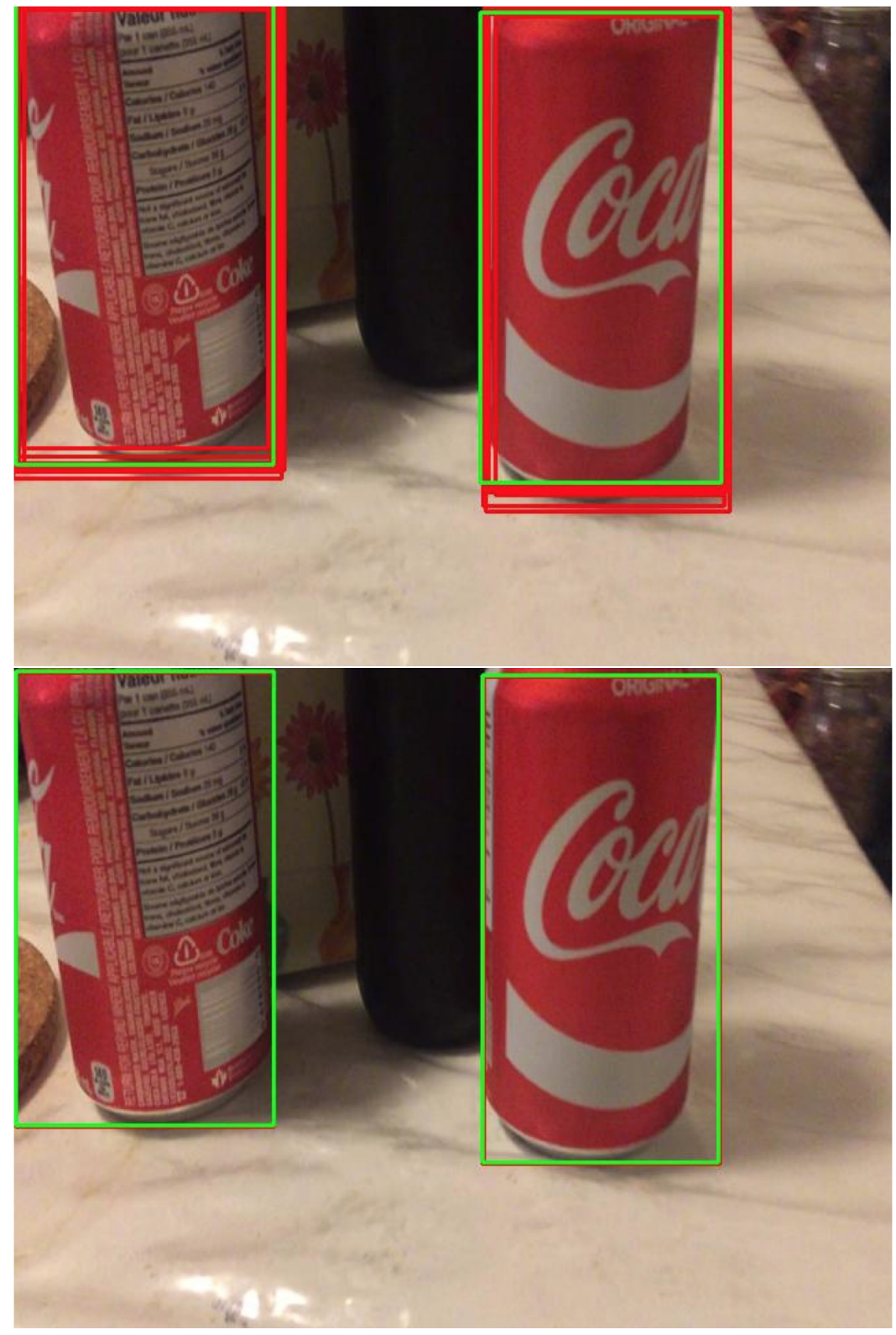

Figure 4.2: Frame 2 with predicted boxes (above) and hand-labeled boxes (below). 

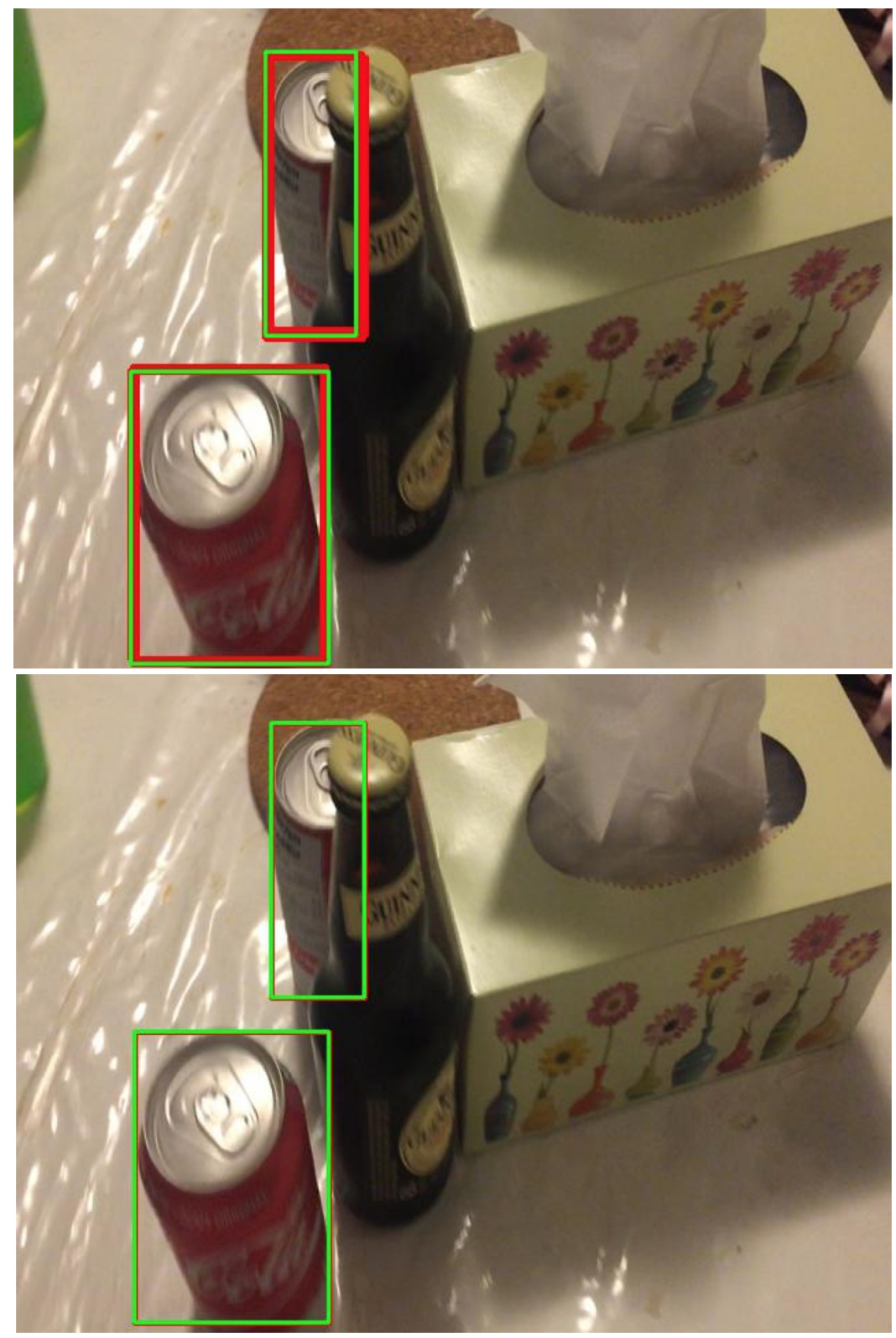

Figure 4.3: Frame 3 with predicted boxes (above) and hand-labeled boxes (below). 

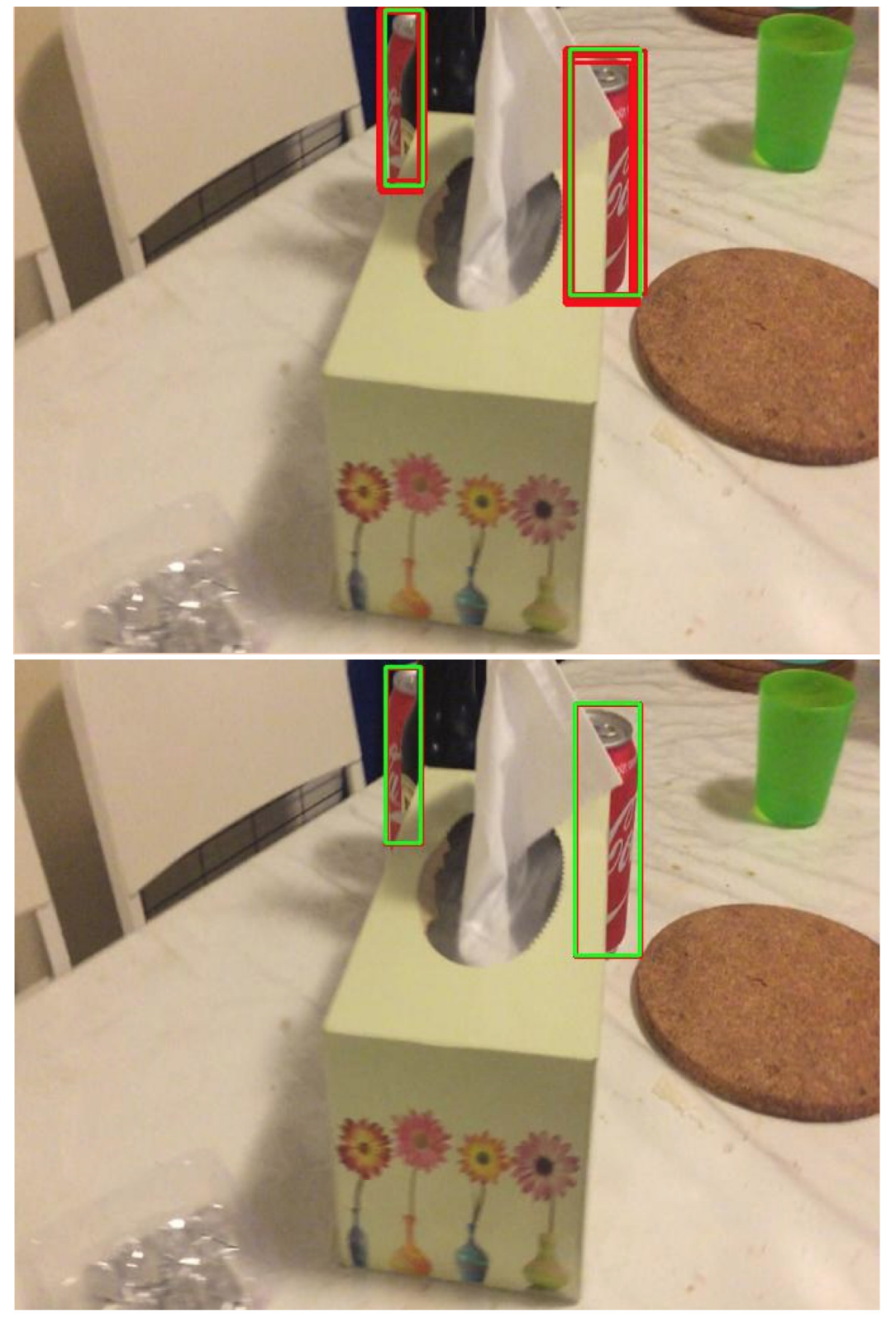

Figure 4.4: Frame 4 with predicted boxes (above) and hand-labeled boxes (below). 

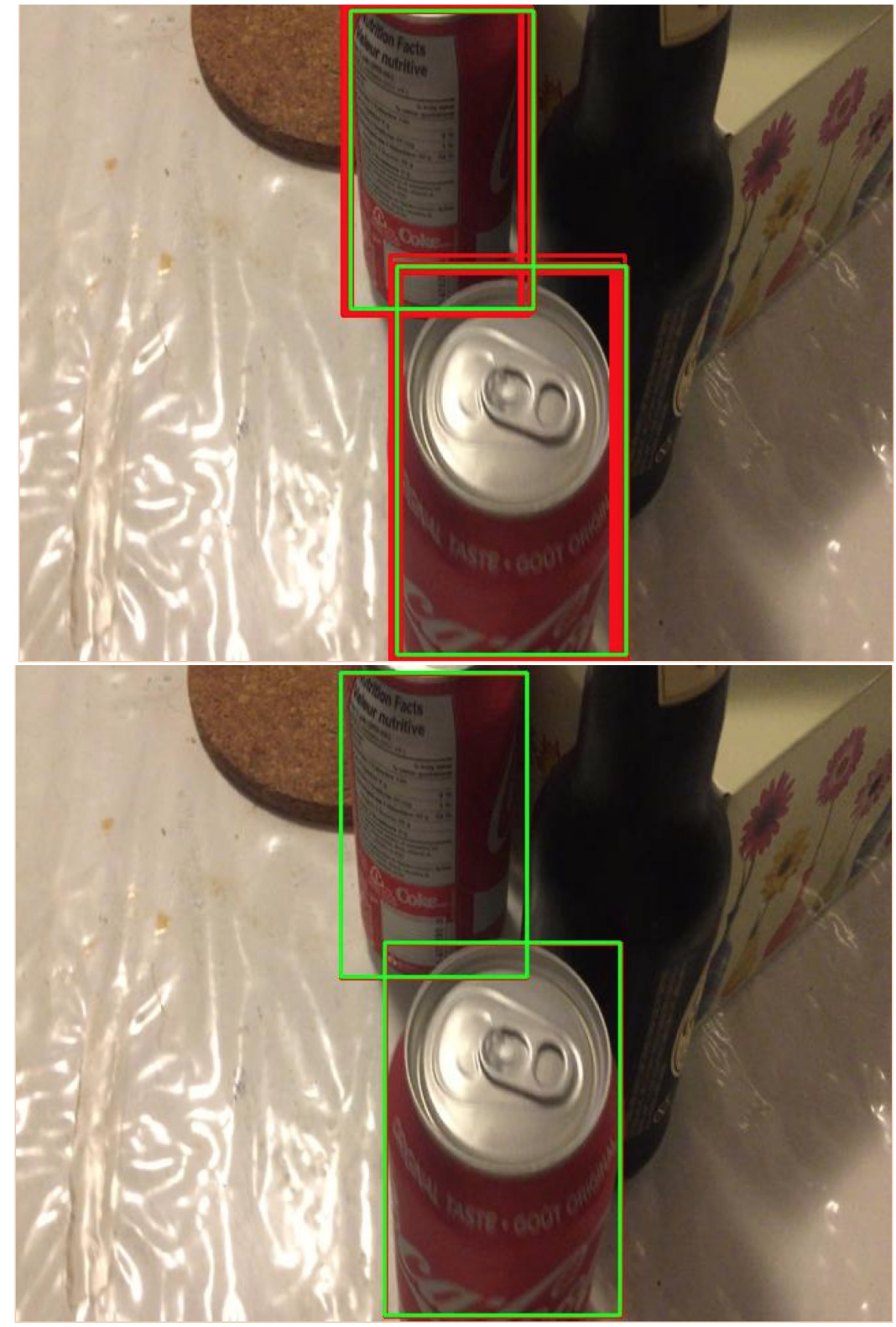

Figure 4.5: Frame 5 with predicted boxes (above) and hand-labeled boxes (below). 


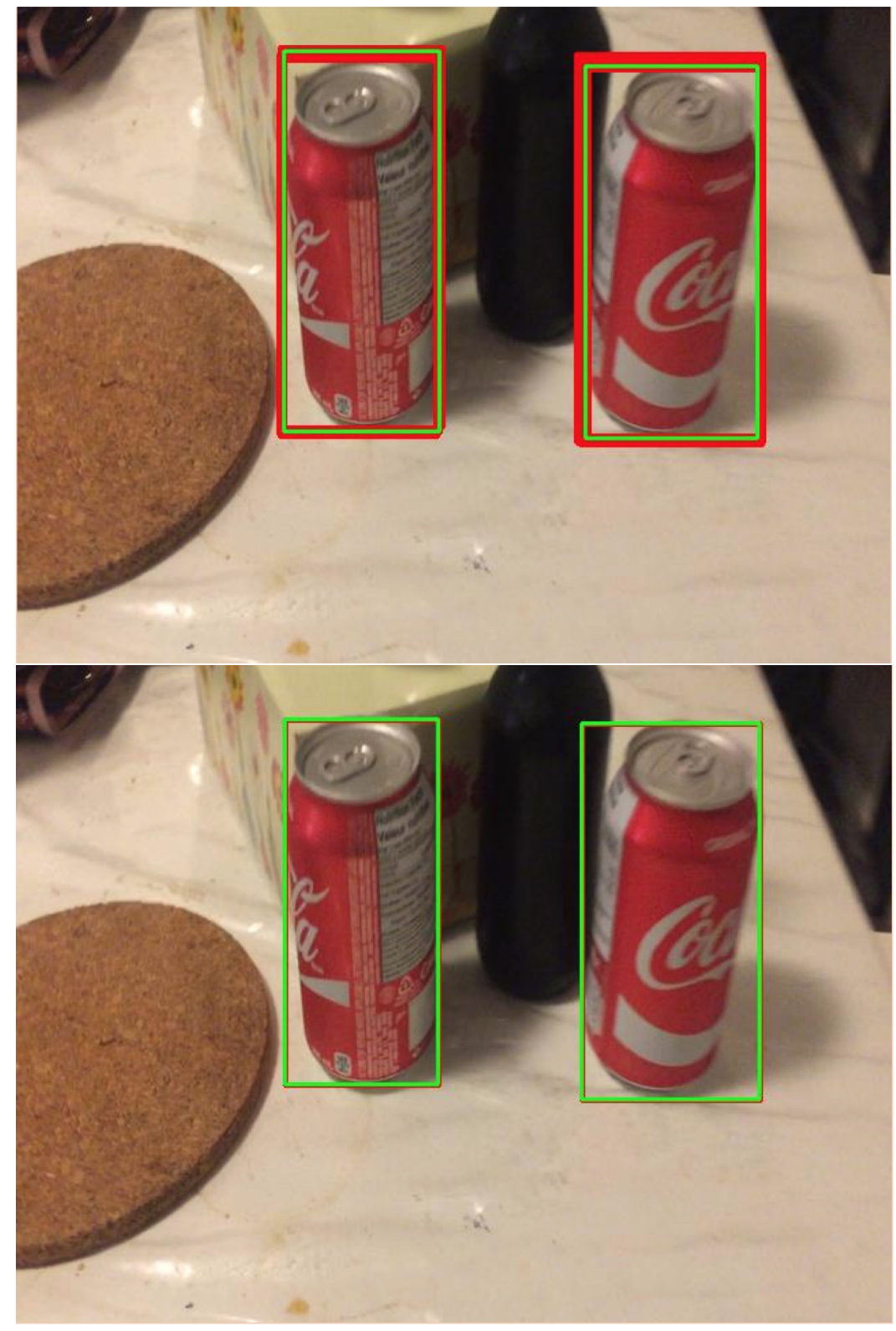

Figure 4.6: Frame 6 with predicted boxes (above) and hand-labeled boxes (below). 

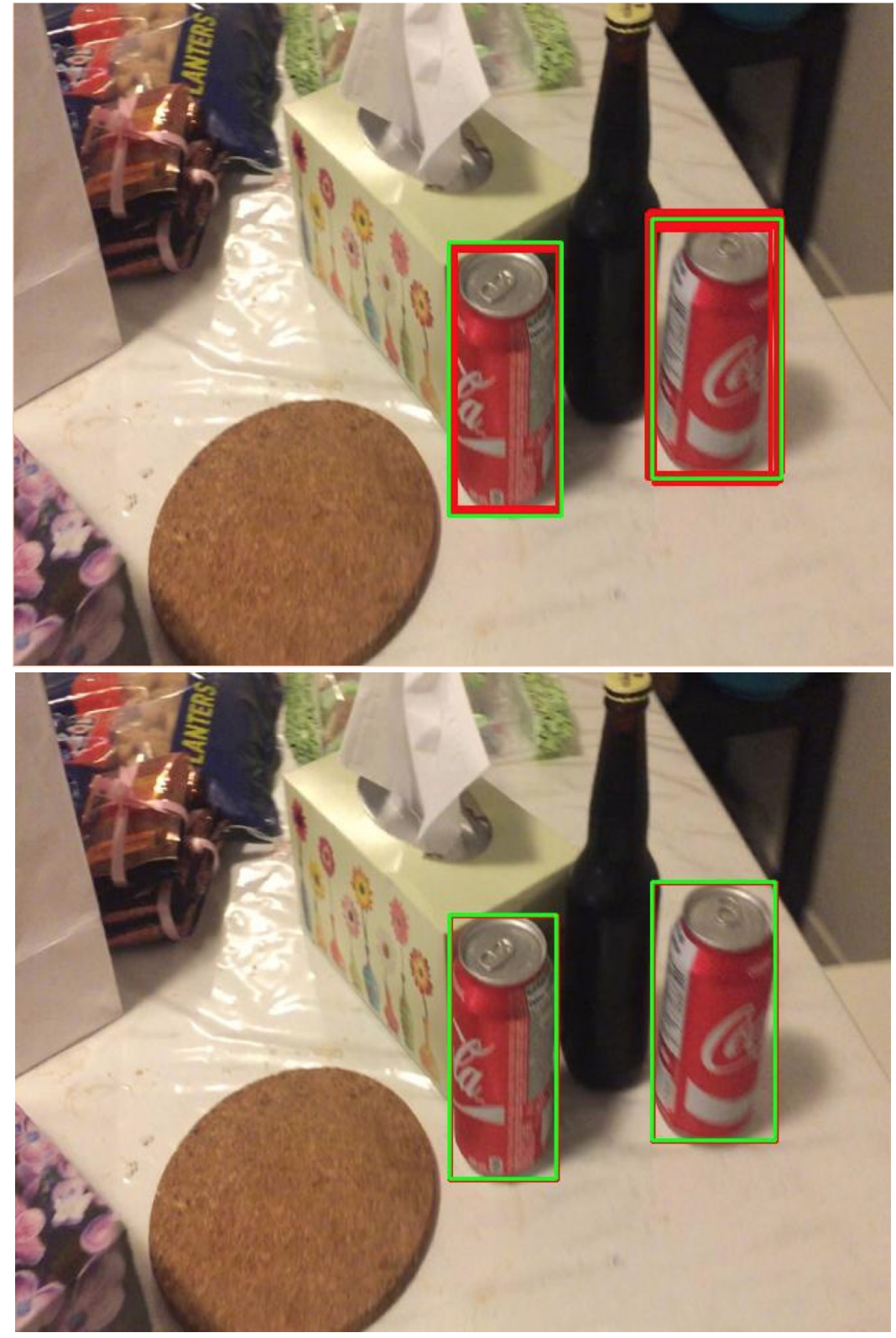

Figure 4.7: Frame 7 with predicted boxes (above) and hand-labeled boxes (below). 
occlusion and scalar variation. Both of the objects are occluded by the tissue box and the bottle. Both of the objects have very small portions visible. Frame 5 shows an example of target object occlusion caused by each other. Frame 6 and Frame 7 show how our network deals with illumination variations, as well as scalar variation.

Using our own video data, we also compare our network with LSTM to a version of the same network without LSTM. The Precision-Recall Curves of the two networks are shown in Figure 4.8. The precision of the network with LSTM is significantly higher than the alternative network, for recall rates below 0.2 .

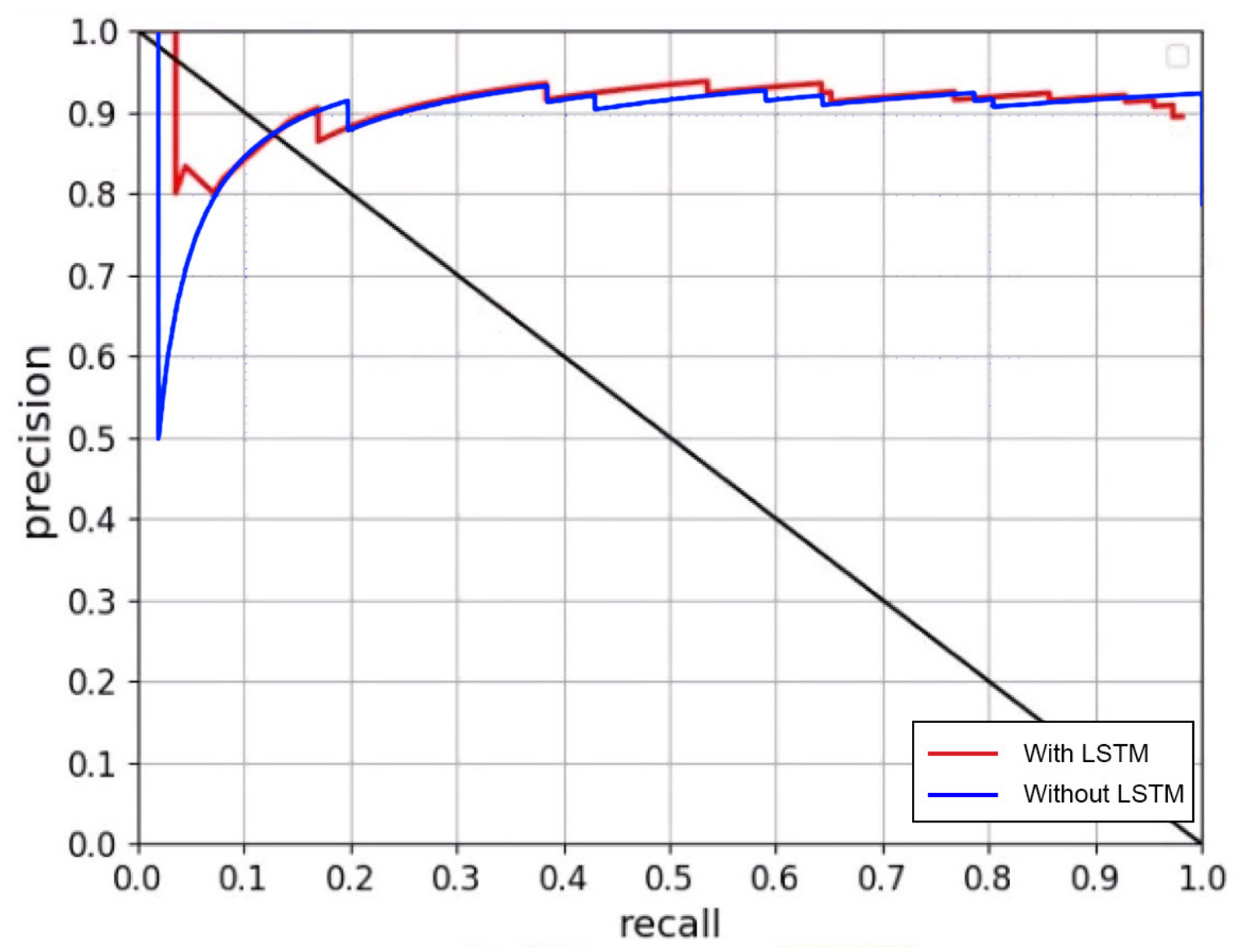

Figure 4.8: Precision-Recall curves without LSTM and with LSTM 
We also compare the results for some of the frames of the video in Figure 4.9. We chose five successive frames. Without LSTM, the system predicted bounding boxes that should not exist, e.g., the one on the label of the beer bottle, since the prediction of the last frame did not influence the prediction of the current frame. We also notice that there is no temporal consistency in the results, since the first and third frames have the extra bounding box, which does not appear in the second frame. In contrast, with the LSTM, although there is a slight variation in the bounding boxes between frames, the location of the predicted boxes is stable, with no unexpected boxes being predicted.
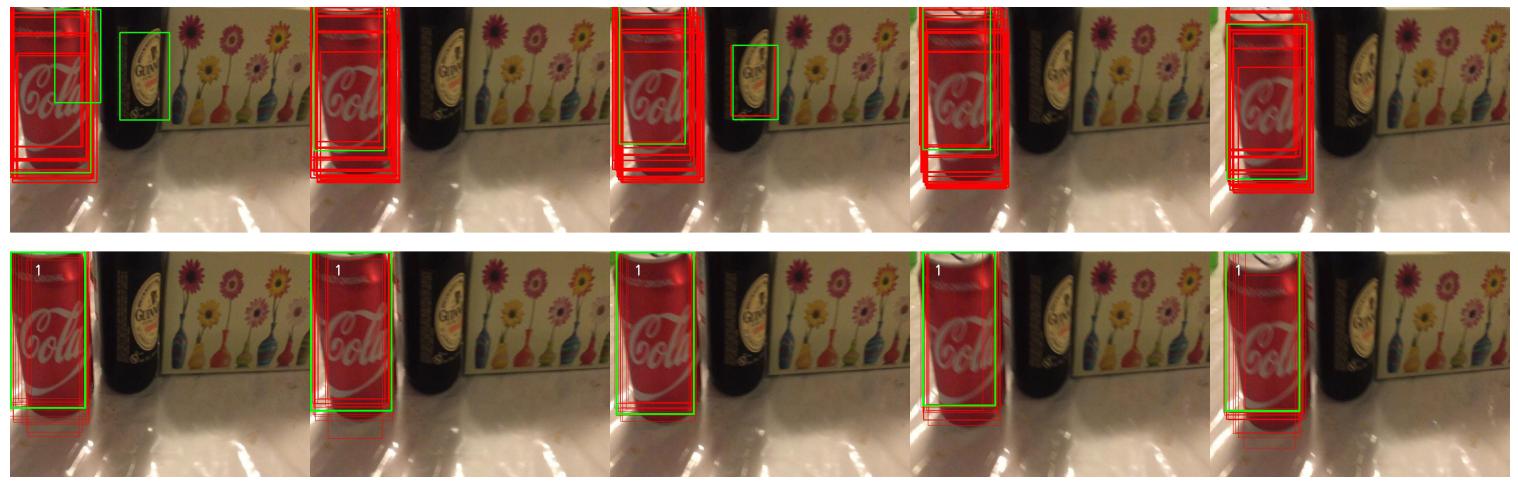

Figure 4.9: Result obtained with the network combined with the LSTM (below), compared to a network without this component (above). Note that the network with LSTM does not detect objects from other classes, e.g., the beer bottle. 


\subsubsection{Results with OTB videos}

We further evaluate our network on a subset of videos of the OTB100 benchmark [5], which was also used in the evaluation of the work of Ning et al. [40]. We select 30 video clips out of the 100 video clips in the benchmark. The 30 videos are Bird1, BlurBody, BlurCar1, BlurCar3, BlurCar4, Boy, Car1, Car4, CarDark, CarScale, Couple, Dancer, Dancer2, David3, Diving, Dog, DragonBaby, Girl2, Gym, Human2, Human3, Human4, Human6, Human7, Human8, Human9, Jogging1, Jogging2, Jump, Singer1, Singer2, Skater, Skater2, Skating1, Skiing, Surfer, Suv, Trans, Walking2, and Woman. We chose these specific videos since they have corresponding classes in the Pascal VOC [14]: Bird, Car, and Person. Thus, it is meaningful to pre-train our network with the images from the Pascal VOC since the same types of objects aim to be detected.

Furthermore, there are around 400 frames in each video. To train the full network with the LSTM, we used some of the frames of each video for training, and the rest for evaluation. The choice was based on the image courtesy of Ning et al. [40] in Figure 4.10. The exact same frame in this figure must be used for evaluation. We made the ratio of training frames and evaluation frame 2:1. We also used the same network for all the classes. The training for each video consisted of 50, 000 iterations, and took around a hour.

Since the OTB100 dataset is meant for visual tracking, there is only one target object in each frame. This is not adequate for training our network, since our goal is to detect all the target types of objects. Thus, we hand-labeled all other objects in the frames to avoid incorrect learning caused by missing ground truth boxes.

In Figure 4.10, we present a qualitative comparison to the work of Ning et al. [40], denoted ROLO (recurrent YOLO). We chose the same frames that they chose in their paper for comparison. Since their work focuses on tracking objects, and the dataset is used for object tracking, only the major object that they intended to track is marked in each frame. For the major object, our results are quite similar.

The task of our network is to detect all objects of the target class. On the other hand, the quantitative analyses Ning et al. [40] performed are for visual trackers. While trackers are used to predict the bounding box in the next frame, these quantitative analyses are not adequate for evaluating the detection of multiple objects. So, we opted not to perform quantitative comparisons with their work. 


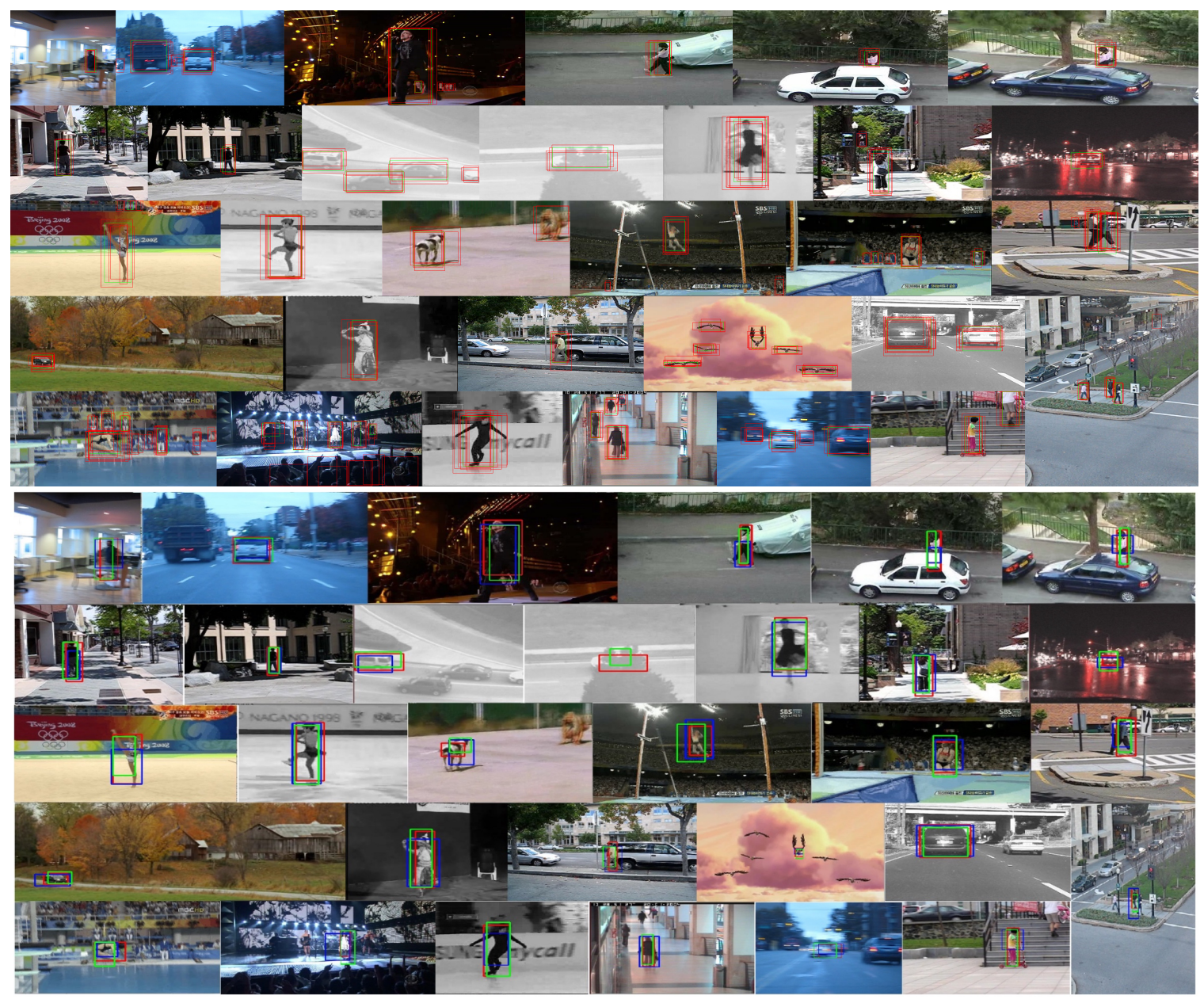

Figure 4.10: Comparison of our results (above) to ROLO's result (below). Image courtesy of Ning et al. [40]. Our network detects several objects for each frame, while Ning's method only tracks one object in each video. 
One limitation of the evaluation discussed above is that we used portions of the same video for training and evaluation. To evaluate our method in a more realistic setting where the training and evaluation videos are completely different, we perform further evaluation with a few selected videos, and compare our network with the LSTM to a version of the network without the LSTM.

Since all the 30 videos selected above belong to 3 classes, we chose two videos of each class, using one video for training and a completely different video for evaluation, with the exception of the video for the bird class. Since there is only one video for this class, we use the first 100 frames for evaluation, and the rest for training. One concern in this choice is that overfitting may occur and perfect prediction rates may be obtained. However, we show in Figure 4.11 that there is enough difference between the portions of the video that we choose for training and evaluation. We observe that there is significant difference in the background of the frames and the fuzziness of the birds' shapes. Moreover, all the training frames show birds flying above the cloud, while all the evaluation frames show birds flying below the clouds.

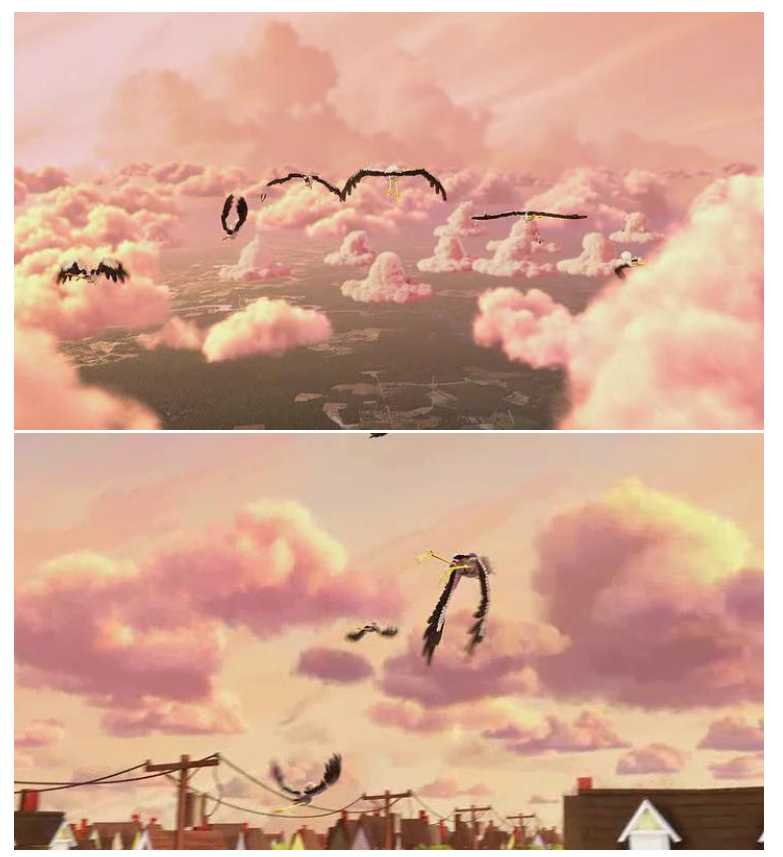

Figure 4.11: Training frame of the Bird class (above) and evaluation frame (below). The background of the frames in the training data is above the clouds, while the background in the evaluation frames is closer to the ground, where roofs and poles appear. 
We pick 3 evaluation frames, and show the results of our network with LSTM and without LSTM in Figure 4.12. Both networks detect one or some of the poles as birds. But we can see that there is a clear tracking in the results of the network with LSTM, while in the results of network without LSTM, there is not temporal consistency in the detected boxes. Meanwhile, the confidences are also quite different. For all the bounding boxes detected by the network with LSTM, their confidences are all higher that 0.91 . The confidences of bounding boxes detected by the network without LSTM can be as low as 0.002. By analyzing the detected boxes, we see that in the frame to the left, both networks detected the bird besides the pole. However, the confidence of the bounding box predicted by the network without LSTM is 0.00106, so it was not marked trustworthy and was not annotated in the frame.

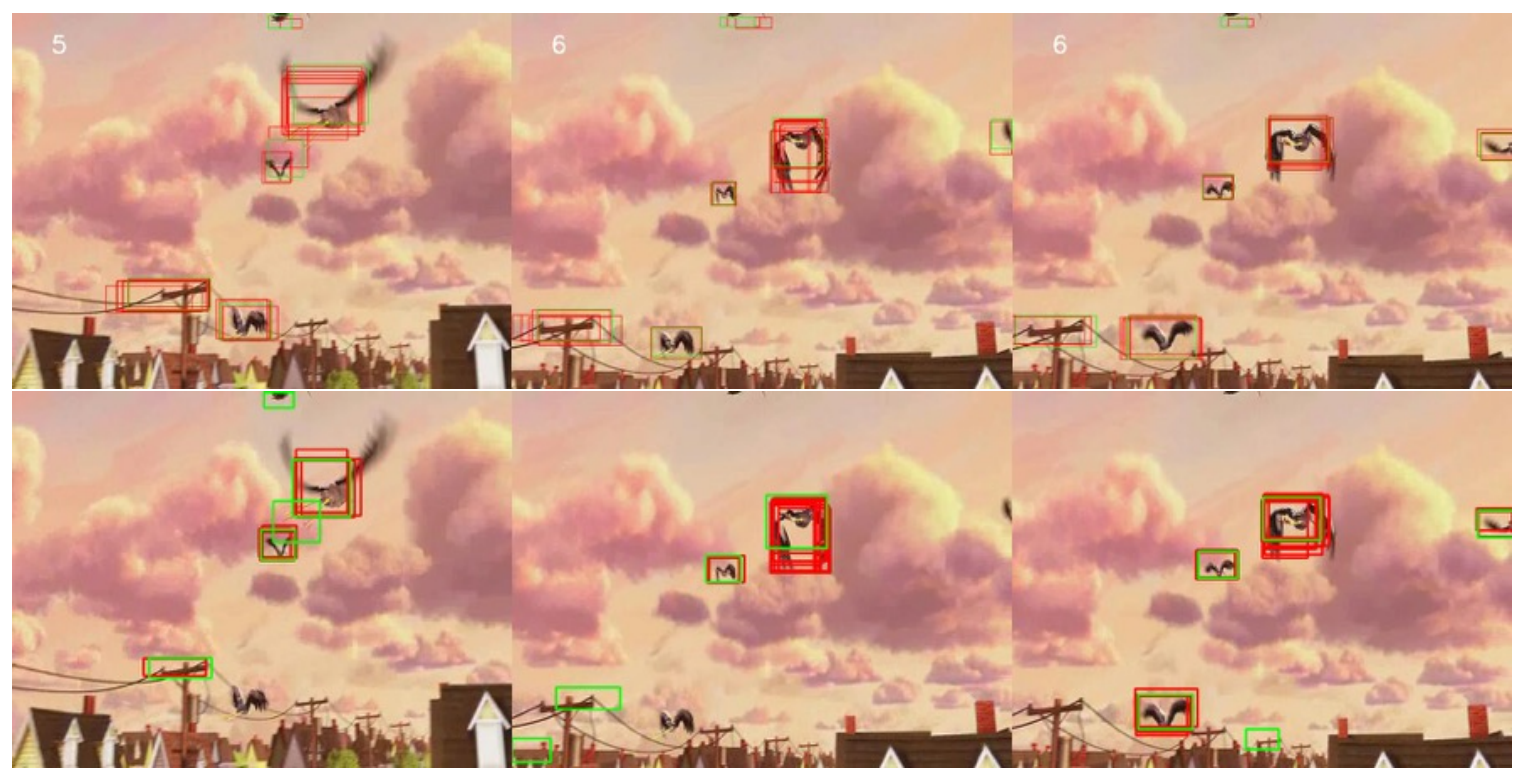

Figure 4.12: Detection results on some frames in a video clip of the Bird class with the LSTM (above) and without LSTM (below). The network with LSTM only detects one object incorrectly in each frame, while the network without LSTM detects two to three objects incorrectly in each frame.

We also compare both networks with precision-recall curves in Figure 4.13. We see that the network with LSTM has better performance than the network without LSTM. The Average Precison for these two networks are 0.83 with LSTM and 0.76 without LSTM. 


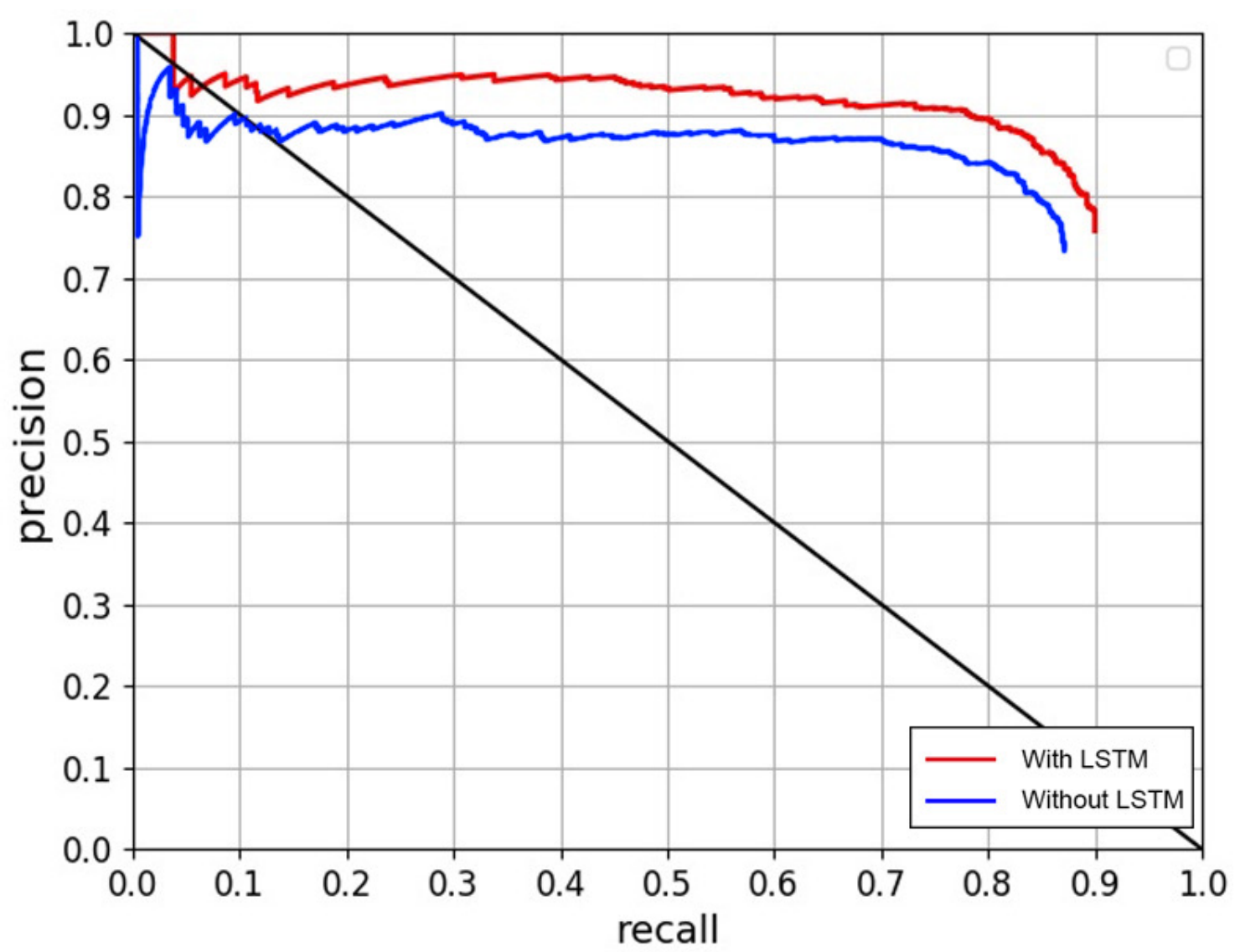

Figure 4.13: Precision-Recall curve for a video of the Bird class with LSTM and without LSTM.

Our network with LSTM takes both the features from the last frame and the newly appeared features of the current frame into consideration when adjusting the feature map of the current frame. Therefore, when an object appears or fades in a video, the network with LSTM is able to detect that. To demonstrate this capability of the network, we show an example involving two sets of successive frames in the evaluation frames of the Bird video clip. The first set shows an example of a bird appearing in a specific frame, whiled the second set shows an example of a bird fading and disappearing. In Figure 4.14, we show that our network with LSTM can catch and track the newly-appeared bird. The five frames appear in sequence, from left to right, top to bottom. There is no bird to the right of the biggest bird in the first frame. In the second frame, a very small part of the bird appears to the right of the biggest bird. As the sequence progresses, the newly-appeared bird becomes bigger 
and bigger. As shown in Figure 4.14, our network with LSTM catches the appearance of this bird in the second frame, and tracks its movement in following frames.

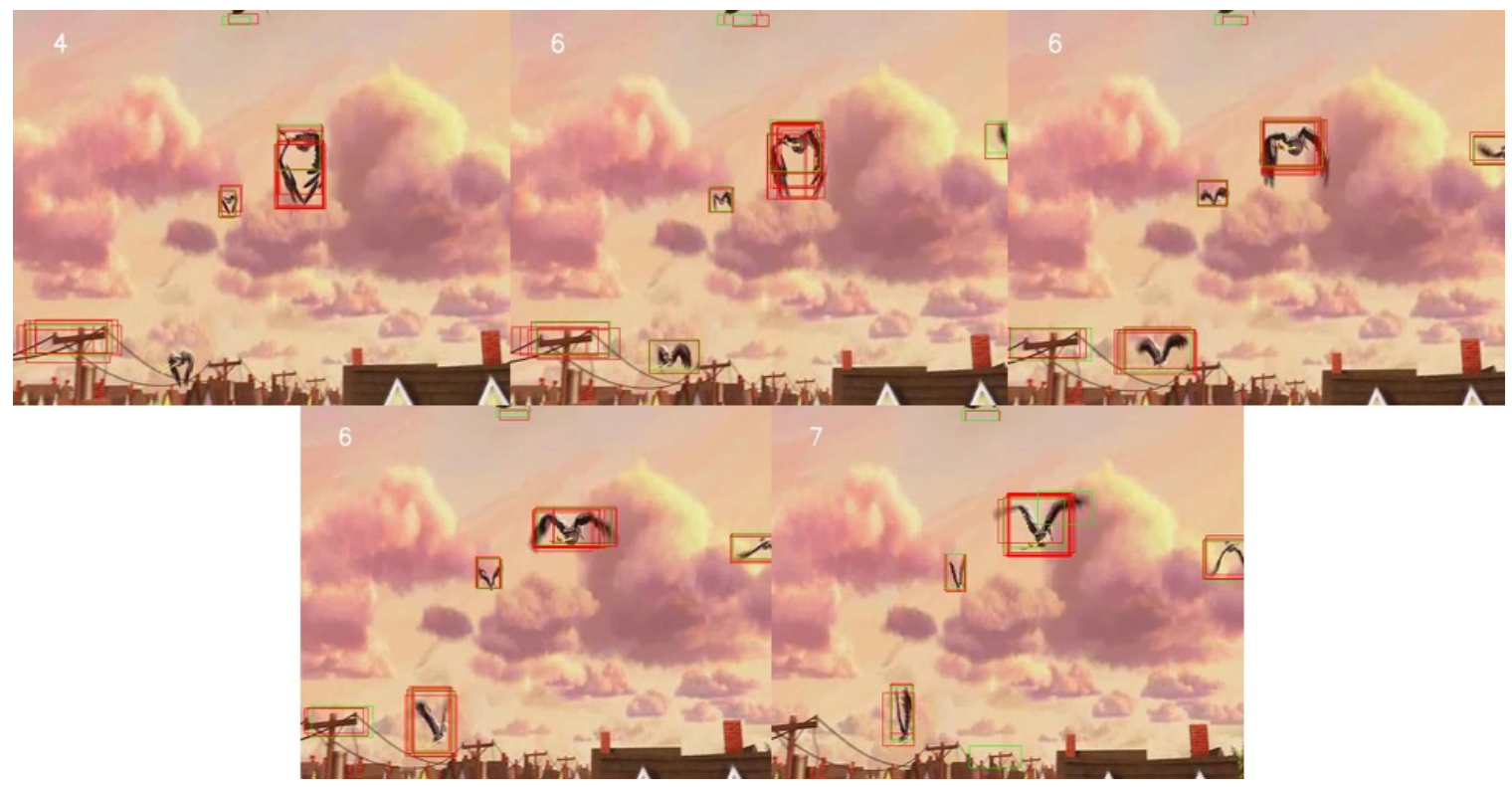

Figure 4.14: An example of our network with LSTM successfully detecting and tracking the newly-appeared bird on the right of the frames.

In Figure 4.15, we show how our network with LSTM tracks and removes a fading object. The four frames are also in sequence, from left to right, top to bottom. The smallest bird on the top-left of the first frame begins to fade in the second frame, and it completely disappears in the third frame. Our network with LSTM removes the bounding box when the bird completely disappears. 


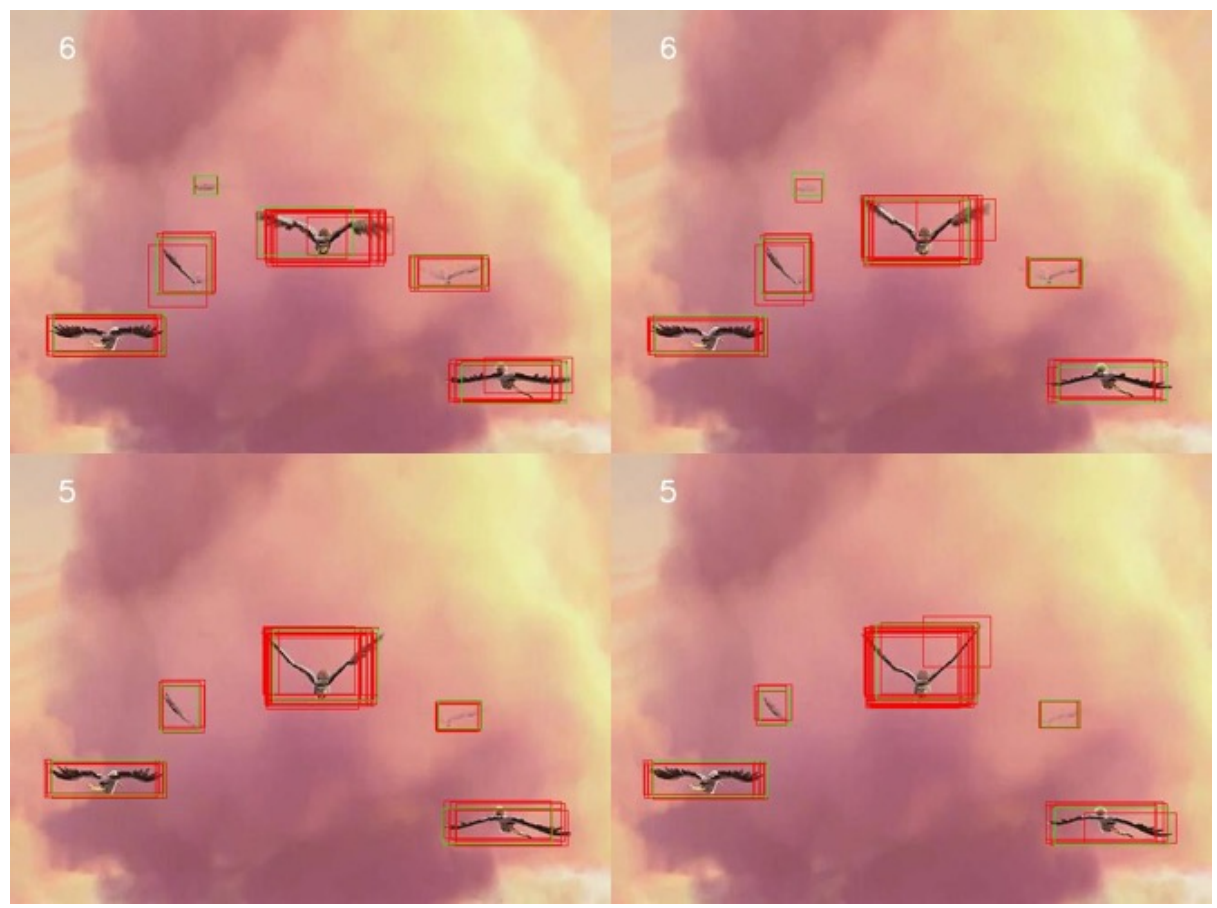

Figure 4.15: An example of our network with LSTM tracking the fading bird on the top-left, and removing the box when the bird fully disappears.

Moreover, for the Person class, we chose the video clips Human7 and Woman. There are 250 frames in Human7, and there are 597 frames in Woman. We chose these two videos because there are some similarities between them. Firstly, only one woman appears in both videos. Secondly, they have similar shapes, and they wear similar clothes. However, the most challenging part is that there are no occlusions in the training video, while there are several occlusions in the evaluation video. We would like to see how the networks with LSTM and without LSTM perform when occlusions happen. We show one frame of each clip in Figure 4.16.

We choose three successive frames in the video clip Woman, and compare the results in Figure 4.17. We can see that the LSTM tracks the objects in the result. Especially, we can see the difference in the second frame, where the network without LSTM predicted two bounding boxes. We further see that the correct bounding box has been predicted, but the confidence of that bounding box is not the highest. So, it was not marked as the final prediction of that frame. 

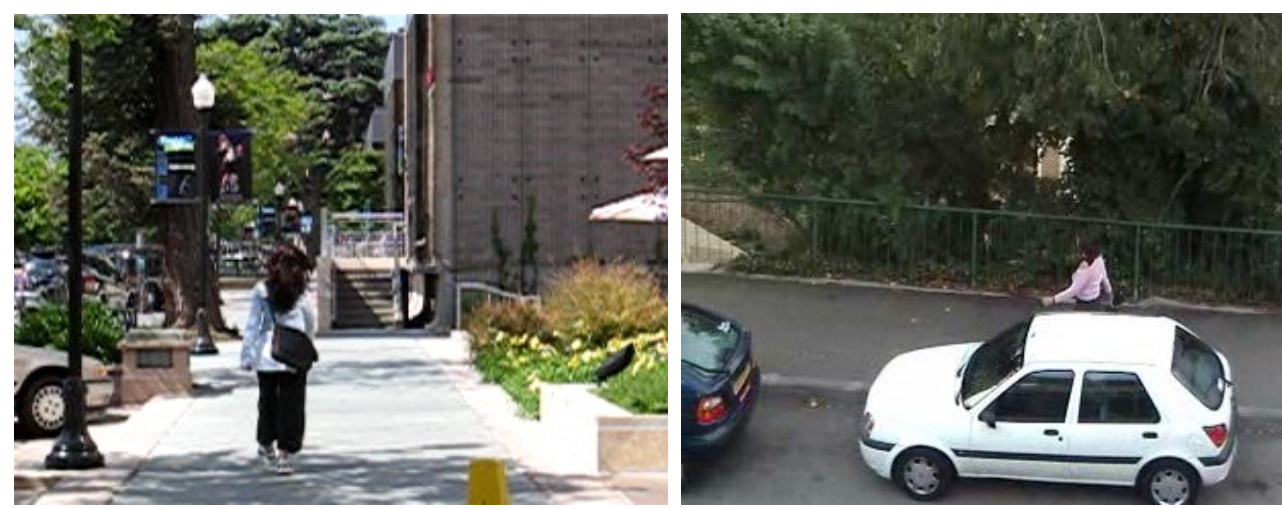

Figure 4.16: Training frame for the person class (left) and evaluation frame (right). The backgrounds of the two videos are different. There is occlusion in the evaluation frame, while there is none in the training frame.

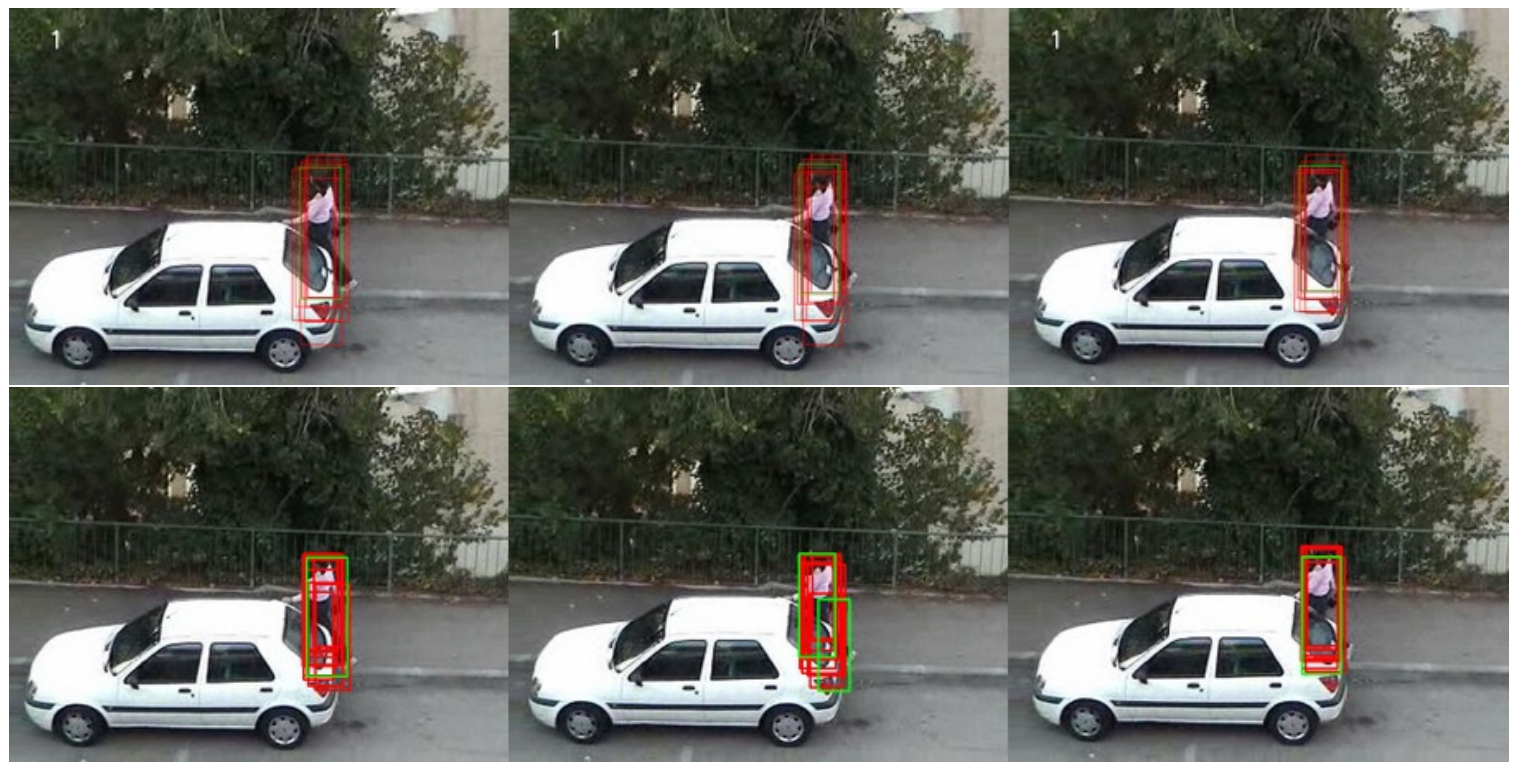

Figure 4.17: Detection results on some frames of the Woman clip with LSTM (above) and without LSTM (below). The detections obtained by the network with LSTM are more consistent, especially for the frame in the middle. 
We also present the Precision-Recall curves of the networks with LSTM and without LSTM in Figure 4.18. We see a clear difference between these two curves. The performance of the network with LSTM is much better than the performance without LSTM. The Average Precision of these two networks are 0.66 and 0.53 , respectively.

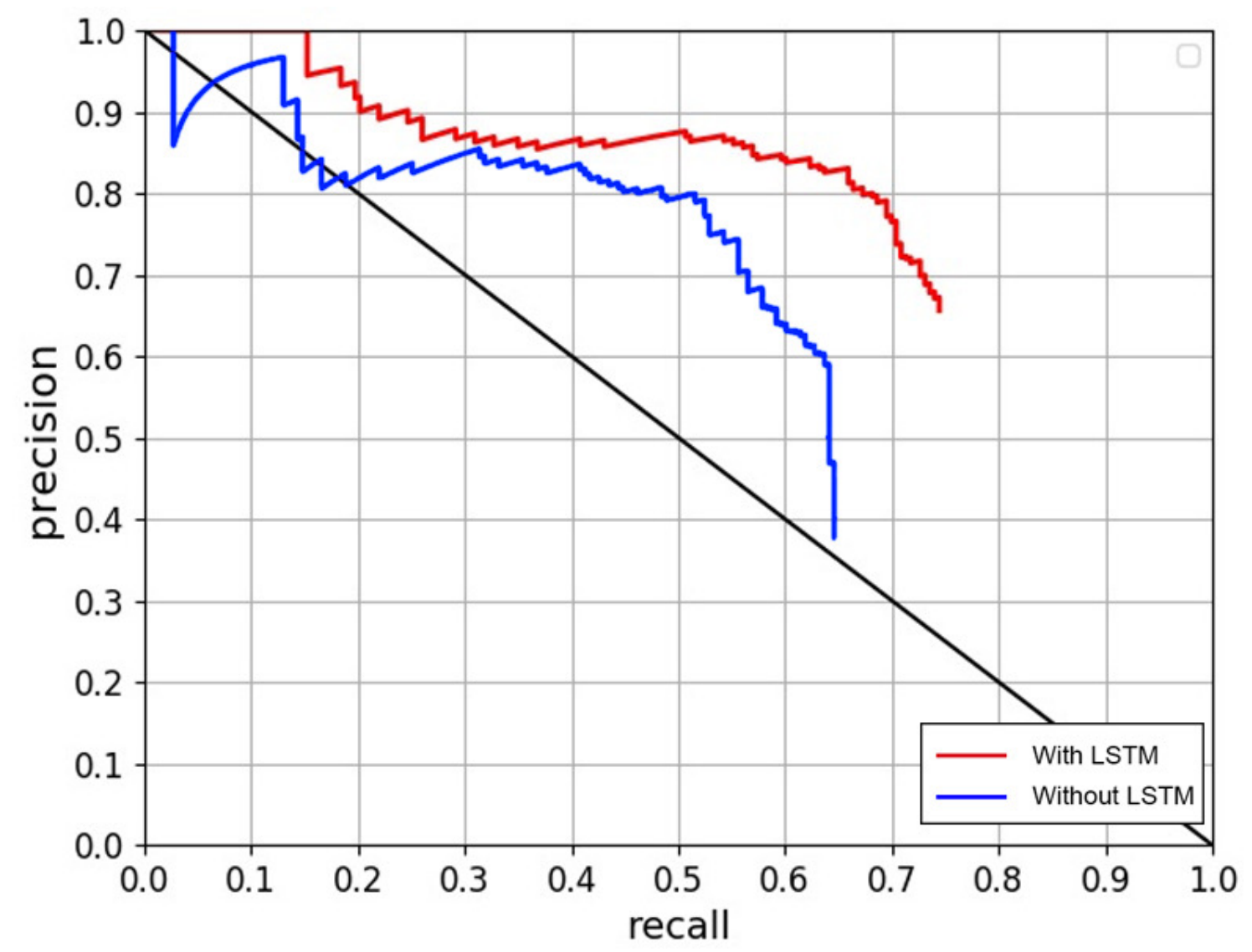

Figure 4.18: Precision-Recall curves on the video clip Woman obtained with LSTM and without LSTM.

For the Car class, we chose the video clip David3 as the training data, and the video clip CarScale as the evaluation data. They both have 252 frames. The video clip David3 is actually used to track a person. But we annotated the cars in this video clip to obtain training data for cars, since most video clips of the Car class in OTB30 [5] are grayscale videos, without color information. We chose CarScale as the evaluation video, to evaluate how the networks with and without LSTM react when scale variations happen, while there is no scale variation in the training data. We show one frame of the training clip and the evaluation clip in Figure 4.19. 


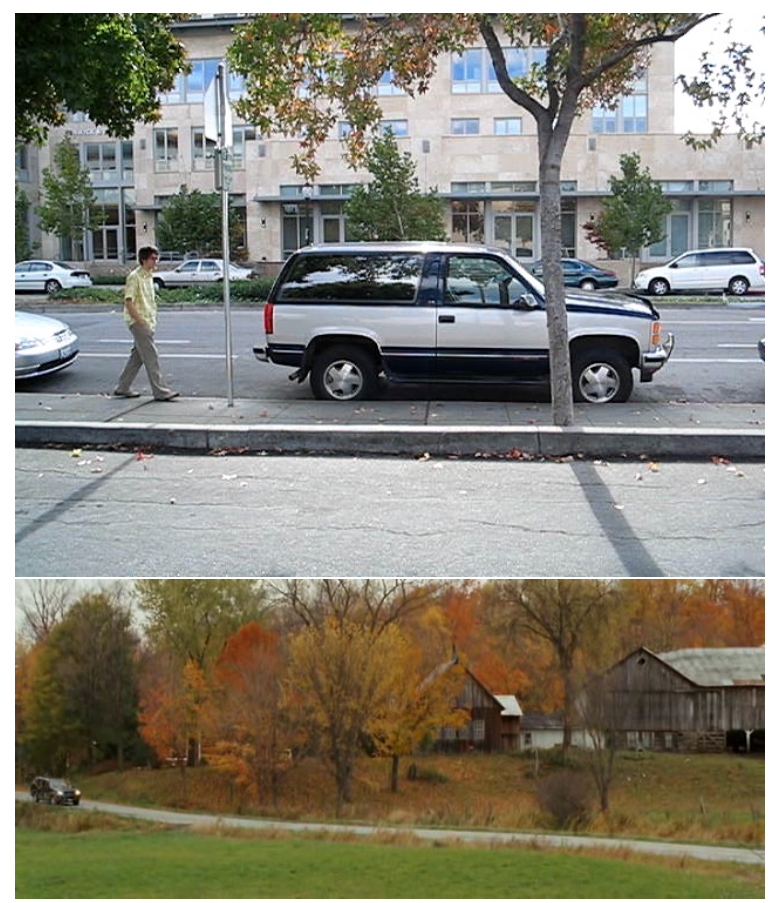

Figure 4.19: Training frame of the Car class (above) and evaluation frame (below). The backgrounds of the frames are different, and there is scale variation in the evaluation frames.

We chose 3 frames of CarScale, and compared the results of the networks with and without LSTM in Figure 4.20. The results are both unsatisfactory due to the large difference in the training clip and the evaluation clip. However, we observe that the results of the network with LSTM are slightly better. 


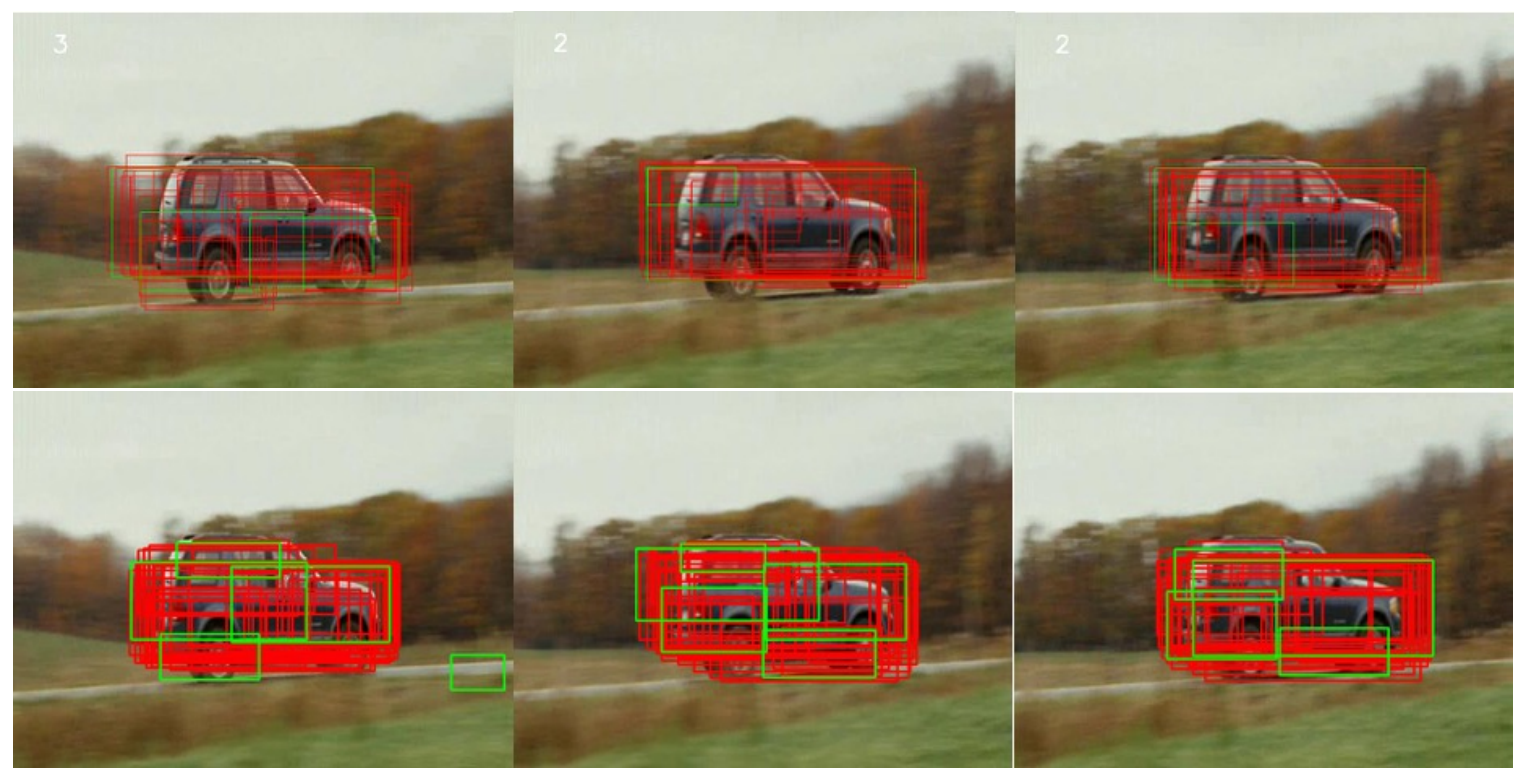

Figure 4.20: Detection results on frames of the video clip CarScale obtained with the LSTM (above) and without the LSTM (below). Both results are not satisfactory. The result obtained by the network with LSTM is slightly more accurate by avoiding the detection on the road given by the network without LSTM.

We show the Precision-Recall curves in Figure 4.21. The Average Precision for the network with LSTM is 0.11, and the Average Precision for the network without LSTM is 0.06 .

We show a training frame with ground-truth boxes in Figure 4.22. The evaluation results are influenced by the training data, because there are many cars annotated in a frame, some with only parts of the car annotated. During the training, the network learned these features as belonging to the class. Therefore, in the evaluation, the car was divided into many parts, which influenced the final results. We would recommend using less crowded videos for training clips in real-life applications. 


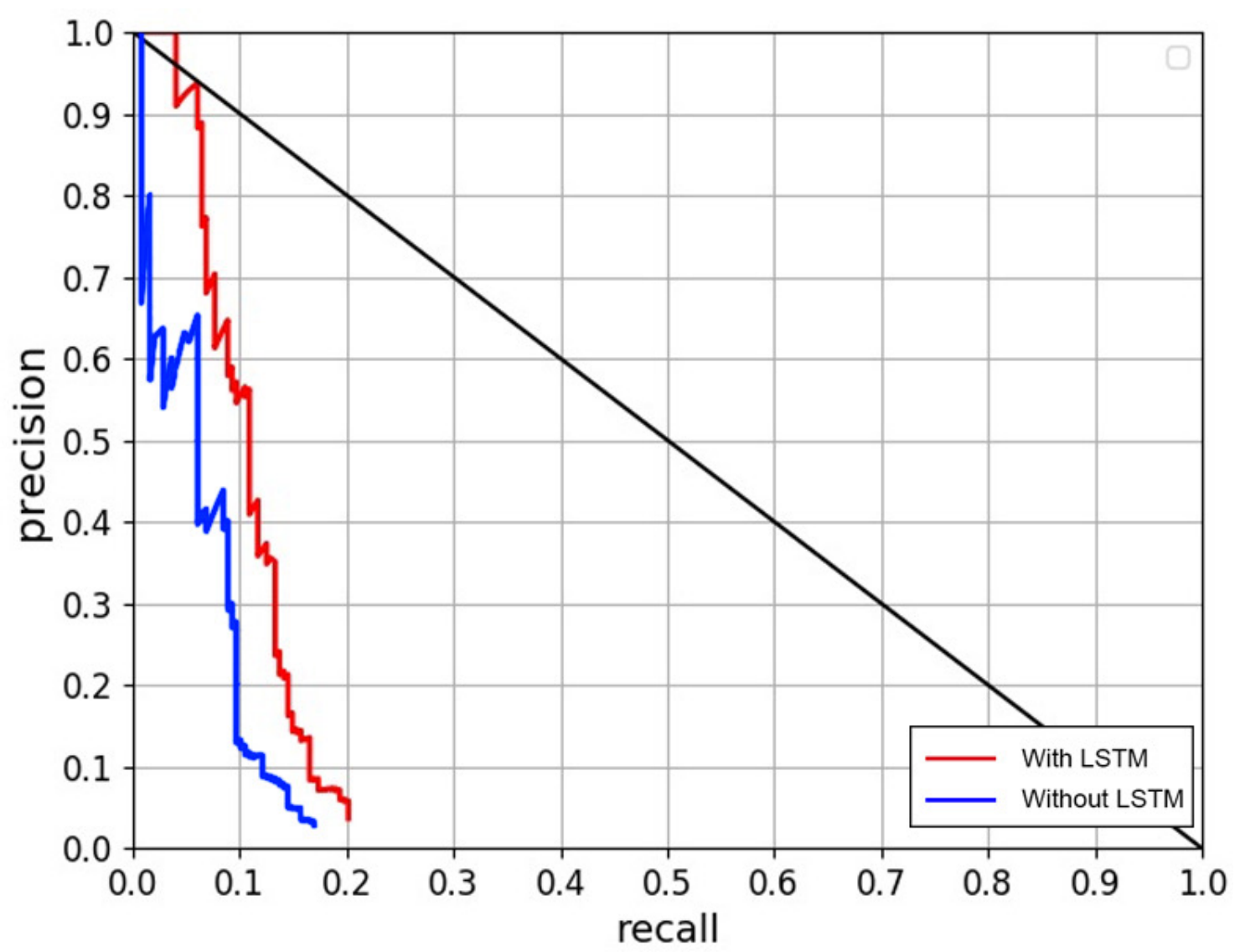

Figure 4.21: Precision-Recall curve on the video clip Carscale with LSTM and without LSTM.

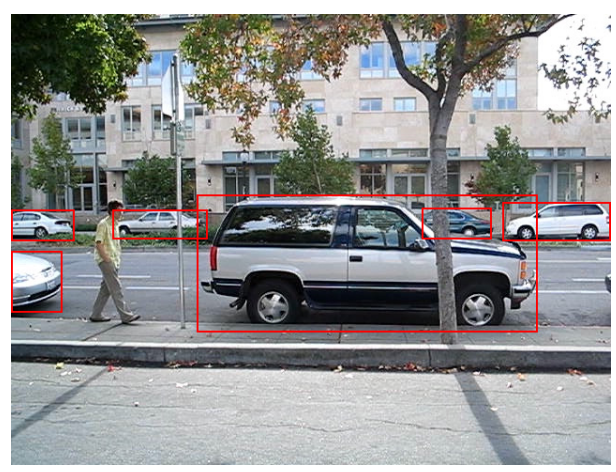

Figure 4.22: Ground-truth boxes in a training frame. 


\subsection{Results with Image Data}

For additional analysis, we also evaluate the results of our network for object detection on single images. This allows us to verify if the performance of our object detector is on par with the state of the art methods. In addition, this allows us to investigate whether our new network structure benefits object detection in images.

We observe that the results are at the level of the state of the art. In these evaluations, we did not use the LSTM parameters we trained with video data, but retrained the LSTM with image data to perform evaluations. For the image datasets, there is no inherent temporal coherence between different images. However, we train the network with the sequence of images as they are listed in the dataset, to benefit from eventual correlations between images.

\subsubsection{Results with Pascal VOC data}

Szegedy et al. [52] compare their neural network with other notable works on the Pascal VOC 2007 dataset. They trained their DetectorNet [52] with VOC 2012 training data, and evaluated it on the VOC 2007 test data, presenting results for each class. We did the training and evaluation on 9 classes of images to compare our work with theirs. The number of training images of each class varies significantly, ranging from 76 (Aeroplane) to around 2,000 images (Person). So, we ran different number of training iterations for each class. For Aeroplane, we ran only 10, 000 iterations. For Person, we ran 60,000 iterations. The training for each class took from half an hour to 3 hours. The comparison of DetectorNet to other works is shown in Figure 4.23. The comparison shows that, for 8 of the 20 classes, their network performed the best. 


\begin{tabular}{|c|c|c|c|c|c|c|c|c|c|c|}
\hline class & aero & bicycle & bird & boat & bottle & bus & car & cat & chair & cow \\
\hline DetectorNet $^{1}$ & .292 & .352 & .194 & .167 & .037 & .532 & .502 & .272 & .102 & .348 \\
\hline Sliding windows $^{1}$ & .213 & .190 & .068 & .120 & .058 & .294 & .237 & .101 & .059 & .131 \\
\hline 3-layer model [19] & .294 & .558 & .094 & .143 & .286 & .440 & .513 & .213 & .200 & .193 \\
\hline Felz. et al. [9] & .328 & .568 & .025 & .168 & .285 & .397 & .516 & .213 & .179 & .185 \\
\hline Girshick et al. [11] & .324 & .577 & .107 & .157 & .253 & .513 & .542 & .179 & .210 & .240 \\
\hline class & table & dog & horse & m-bike & person & plant & sheep & sofa & train & tv \\
\hline DetectorNet $^{1}$ & .302 & .282 & .466 & .417 & .262 & ב.103 & .328 & .268 & .398 & .470 \\
\hline Sliding windows $^{1}$ & .110 & .134 & .220 & .243 & .173 & .070 & .118 & .166 & .240 & .119 \\
\hline 3-layer model [19] & .252 & .125 & .504 & .384 & .366 & .151 & .197 & .251 & .368 & .393 \\
\hline Felz. et al. [9] & .259 & .088 & .492 & .412 & .368 & .146 & .162 & .244 & .392 & .391 \\
\hline Girshick et al. [11] & .257 & .116 & .556 & .475 & .435 & .145 & .226 & .342 & .442 & .413 \\
\hline
\end{tabular}

Figure 4.23: Comparison of DetectorNet to other object detection works, in terms of mean Average Precision (mAP). Image courtesy of Szegedy et al. [52].

The comparison of our work and the best results in the table above is shown in Table 4.1. For most of the classes, our network performs better. For the Person and Sofa classes, our results are very close to the best of the former result in Figure 4.23. For the Bird and Boat classes, the mean Average Precision (mAP) of our network is 0.03 to 0.04 more than the best of former works shown in Figure 4.23. For the Bus and Dog classes, our result is around 0.1 better than the best of former works in Figure 4.23. However, we have worse results on the Train, Bus, and Aeroplane classes.

\begin{tabular}{|l|l|l|}
\hline Classes & Best results in [52] & Our results \\
\hline Airplane & .328 & .266 \\
\hline Bird & .194 & .231 \\
\hline Boat & .168 & .196 \\
\hline Bottle & .286 & .294 \\
\hline Bus & .532 & .446 \\
\hline Dog & .282 & .384 \\
\hline Person & .435 & .444 \\
\hline Sofa & .342 & .344 \\
\hline Train & .442 & .323 \\
\hline
\end{tabular}

Table 4.1: Comparison of our work to the best-performing method mentioned in [52]. The evaluation measure is the mean Average Precision (mAP). 
One possible reason for the worse results in these classes is that we ran less iterations when training these three classes. Also, the VOC datasets have images with very few features, and also not $100 \%$ correct bounding boxes. These factors may also influence our results.

Figure 4.24 and Figure 4.25 show two graphs comparing our recall and precision with the recall and precision of Szegedy et al. [52]. We have similar curves for the Bus class, although our recall-precision curve drops earlier than their curve. For the Bird class, their curve drops sharply around a recall of 0.05 , whereas our curve drops sharply around a recall of 0.2. The average precision we obtain for the Bird class is superior than their result. But for the Bus class, their network performed the best. 

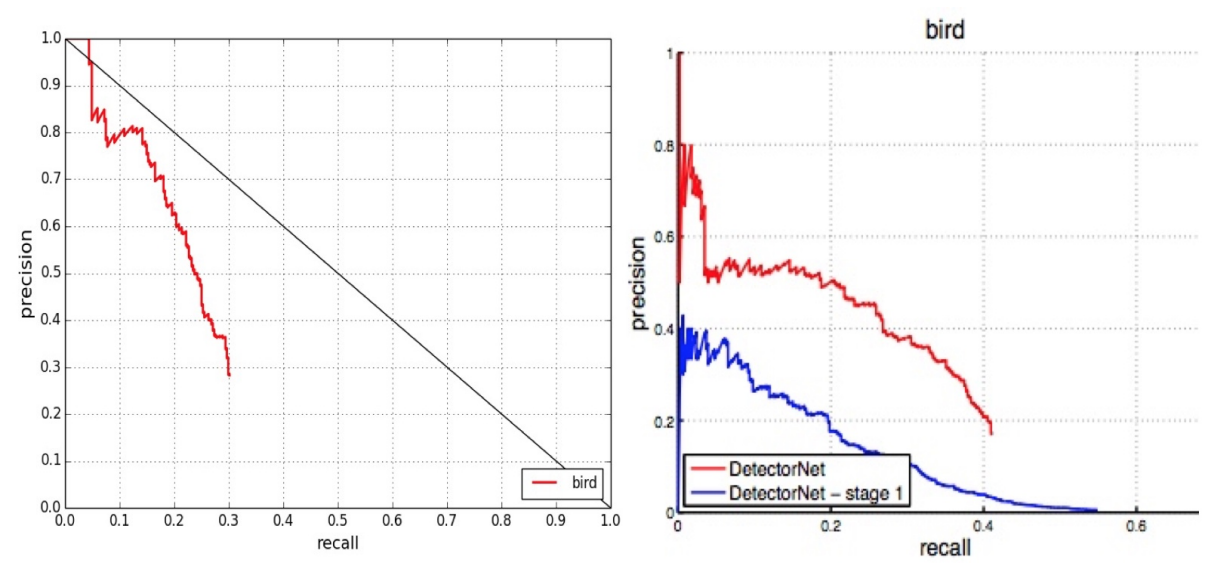

Figure 4.24: Comparison of the Recall and Precision for the Bird class: our network (left), DetectorNet (right, image courtesy of Szegedy [52]).
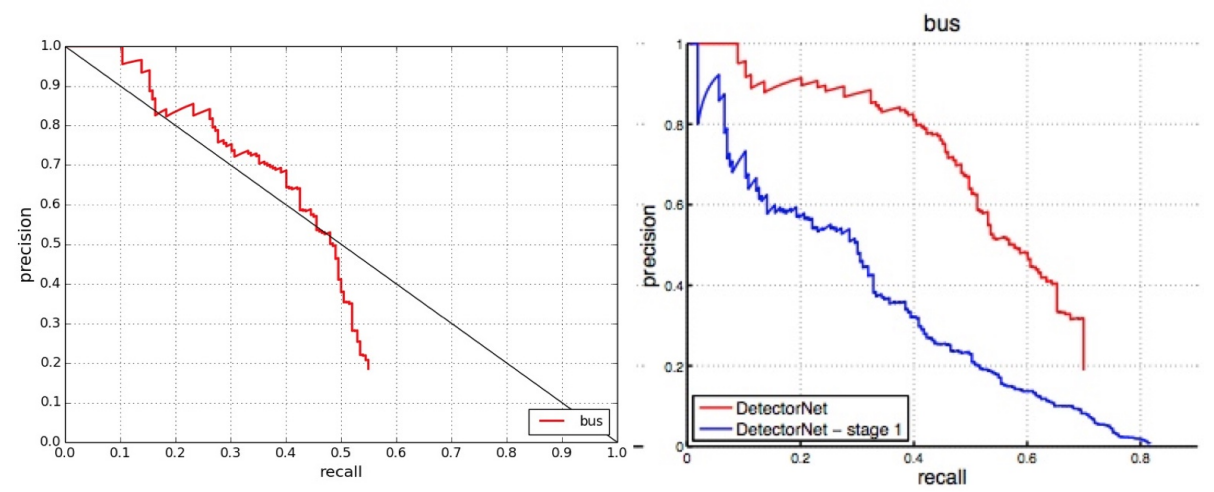

Figure 4.25: Comparison of the Recall and Precision for the Bus class: our network (left), DetectorNet (right, image courtesy of Szegedy [52]).

\subsubsection{Results with ImageNet Data}

ImageNet [45] has held the ILSVRC from 2010 to 2017, providing a dataset of 1,000 classes that is publicly available. We used their dataset of 200 classes for object detection, as well as the object annotations for these classes of objects. The number of images in each class also varies significantly. We used the ILSVRC 2015 training and testing datasets for training and evaluation respectively. We trained 15,000 iterations for each class, which took us four hours. We obtained a mean Average 
Precision (mAP) of $72.2 \%$. The results for the object detection task in the ILSVRC 2017 are listed in Table 4.2 [4]. Our mAP is $0.1 \%$ less than the state of the art performance. However, note that this comparison is only approximate, since we use the ILSVRC 2015 datasets in our evaluation, while the ILSVRC 2017 provides a new set of test images. The ILSVRC training data has not changed since 2014, but their testing data has been partially updated every year. Although our performance is not superior, our system yields reasonably good results. The mAP of our network running on ImageNet [45] data is better than the result of the Pascal VOC data. Because the objects in the training images are large and normally in the center of the image, it is easier for the network to extract features from images of this dataset.

\begin{tabular}{|l|l|l|}
\hline Team Name & Number of Object Categories Won & mean AP \\
\hline BDAT & 85 & 0.731392 \\
\hline BDAT & 65 & 0.732227 \\
\hline BDAT & 20 & 0.723712 \\
\hline DeepView(ETRI) & 10 & 0.593084 \\
\hline NUS-Qihoo_DPNs(DET) & 9 & 0.656932 \\
\hline
\end{tabular}

Table 4.2: The results of the ILSVRC2017 object detection task with provided training data [4]. 


\section{Chapter 5}

\section{Conclusion}

In this thesis, we proposed a new type of network for recognizing the bounding boxes of objects in videos, which combines object detection with tracking. We use GoogLeNet [51] as a feature extractor to extract features of video frames or images. Then, we use an LSTM network to further adjust the features extracted by GoogLeNet based on the context of features extracted from the last video frame. At last, we employ the last layers of the Overfeat network as a classifier and regressor to obtain the predicted confidences and predicted bounding boxes of objects.

Although our work shares similarities with the work of Ning et al. [40], we take into account image features for tracking, rather than only the bounding boxes of objects. Furthermore, the problem targeted by their tool is slightly different than ours, more intended for pure tracking than object detection + tracking as in our work.

We showed with qualitative examples that the results of our proposed network with the LSTM component has a better accuracy for object detection on frames where occlusions and background clutter occur, when compared to the same network without accounting for temporal coherency with an LSTM. The consideration of temporal coherency also leads to more consistent bounding boxes across frames. At the same time, the network obtains object detection results with accuracy similar to the state-of-the-art methods for single images. Finally, the network is able to process videos at the rate of 6 frames per second, providing a suitable solution for the intended application of creating interactive videos. 


\section{Chapter 6}

\section{Future Work}

Although we obtained promising result so far, in the future, we would like to perform a more thorough evaluation of our method to demonstrate its robustness. This would include training and testing with a larger dataset of videos, including more diversity in the appearance of objects from a same class, and also more diversity of classes. At the same time, it is important to provide further quantitative experiments.

It would also be interesting to investigate alternative architectures, tracking solutions, and training schemes for our network. For architectures, it would be insightful to demonstrate the influence that the number of layers of the ConvNet and number of cells in the LSTM have on the accuracy of the results. For tracking, we could investigate the use of a Kalman filter as an alternative solution for tracking the features generated by the ConvNet, since such a filter would be faster than our current solution using an LSTM. In terms of training, it would be interesting to verify if the training procedure can be broken down into even smaller tasks, for example, also training the classifier and regressor individually, so that the network can be trained with larger amounts of data.

Furthermore, we investigated only the object detection aspect of the creation of interactive videos. Further work could also focus on the interaction component of these videos. The hope is to draw an audiences' attention by providing intriguing interactions. Thus, we need proposals for other possible types of interactions that can be introduced into videos, methods for creating such interactions, and some empirical study to demonstrate their usability. 


\section{List of References}

[1] "Cifar-10 and cifar-100 datasets." [Online]. Available: https://www.cs.toronto. edu/ kriz/cifar.html

[2] "Cs231n convolutional neural networks for visual recognition." [Online]. Available: http://cs231n.github.io/convolutional-networks/

[3] "Imagenet." [Online]. Available: http://www.image-net.org/

[4] "Large scale visual recognition challenge 2017 (ilsvrc2017)." [Online]. Available: http://image-net.org/challenges/LSVRC/2017/results

[5] "Visual tracker benchmark." [Online]. Available: http://cvlab.hanyang.ac.kr/ tracker_benchmark/datasets.html

[6] "Vot 2017 benchmark." [Online]. Available: http://www.votchallenge.net/ $\operatorname{vot} 2017 /$

[7] M. Abadi, A. Agarwal, P. Barham, E. Brevdo, Z. Chen, C. Citro, G. S. Corrado, A. Davis, J. Dean, M. Devin et al., "Tensorflow: Large-scale machine learning on heterogeneous distributed systems," in Proc. USENIX Conference on Operating Systems Design and Implementation, 2016, pp. 265-283.

[8] A. M. Andrew, "Backpropagation," Kybernetes, vol. 30, no. 9-10, pp. 1110$1116 ; 1117 ;, 2001$.

[9] S. Arora, A. Bhaskara, R. Ge, and T. Ma, "Provable bounds for learning some deep representations," in International Conference on Machine Learning, 2014, pp. $584-592$.

[10] V. Badrinarayanan, A. Kendall, and R. Cipolla, "SegNet: A deep convolutional encoder-decoder architecture for image segmentation," IEEE Transactions on Pattern Analysis and Machine Intelligence, vol. 39, no. 12, pp. 2481-2495, 2017.

[11] S. Behnke, Hierarchical neural networks for image interpretation. Springer Science \& Business Media, 2003, vol. 2766.

[12] J. L. Elman, "Finding structure in time," Cognitive science, vol. 14, no. 2, pp. 179-211, 1990.

[13] D. Erhan, C. Szegedy, A. Toshev, and D. Anguelov, "Scalable object detection using deep neural networks," in Proceedings of the IEEE Conference on Computer Vision and Pattern Recognition, 2014, pp. 2147-2154. 
[14] M. Everingham, L. Van Gool, C. K. I. Williams, J. Winn, and A. Zisserman, "The pascal visual object classes (voc) challenge," International Journal of Computer Vision, vol. 88, no. 2, pp. 303-338, jun 2010.

[15] K. Fukushima and S. Miyake, "Neocognitron: A self-organizing neural network model for a mechanism of visual pattern recognition," in Competition and cooperation in neural nets. Springer, 1982, pp. 267-285.

[16] R. Girshick, J. Donahue, T. Darrell, and J. Malik, "Rich feature hierarchies for accurate object detection and semantic segmentation," in Proceedings of the IEEE conference on computer vision and pattern recognition, 2014, pp. 580-587.

[17] A. Graves, A.-r. Mohamed, and G. Hinton, "Speech recognition with deep recurrent neural networks," in Acoustics, speech and signal processing (icassp), 2013 ieee international conference on. IEEE, 2013, pp. 6645-6649.

[18] Y. Guo, Y. Liu, A. Oerlemans, S. Lao, S. Wu, and M. S. Lew, "Deep learning for visual understanding," Neurocomputing, vol. 187, no. C, pp. 27-48, 2016.

[19] R. I. Hammoud, Interactive video: algorithms and technologies. Springer, 2006.

[20] D. O. Hebb, The organization of behavior: A neuropsychological theory. Psychology Press, 2005.

[21] G. Hinton, N. Srivastava, and K. Swersky, Neural Networks for Machine Learning, 2017. [Online]. Available: http://www.cs.toronto.edu/ tijmen/ csc321/slides/lecture_slides_lec6.pdf

[22] S. Hochreiter and J. Schmidhuber, "Long short-term memory," Neural computation, vol. 9, no. 8, pp. 1735-1780, 1997.

[23] K. Hornik, G. Dorffner, H. Bischof, and S. O. service), Artificial Neural Networks ICANN 2001: International Conference Vienna, Austria, August 21, 2001 Proceedings. Berlin, Heidelberg: Springer Berlin Heidelberg, 2001, vol. 2130.;2130;.

[24] D. H. Hubel and T. N. Wiesel, "Receptive fields, binocular interaction and functional architecture in the cat's visual cortex," The Journal of physiology, vol. 160, no. 1, pp. 106-154, 1962.

[25] B. Huval, T. Wang, S. Tandon, J. Kiske, W. Song, J. Pazhayampallil, M. Andriluka, P. Rajpurkar, T. Migimatsu, R. Cheng-Yue et al., "An empirical evaluation of deep learning on highway driving," arXiv preprint arXiv:1504.01716, 2015 .

[26] A. Karpathy and L. Fei-Fei, "Deep visual-semantic alignments for generating image descriptions," IEEE Transactions on Pattern Analysis and Machine Intelligence, vol. 39, no. 4, pp. 664-676, 2017.

[27] D. P. Kingma and J. Ba, "Adam: A method for stochastic optimization," Proceedings of the 3rd International Conference on Learning Representations (ICLR), 2014. 
[28] A. Krizhevsky and G. Hinton, Learning multiple layers of features from tiny images, 2009, vol. 2.

[29] A. Krizhevsky, I. Sutskever, and G. E. Hinton, "Imagenet classification with deep convolutional neural networks," in Advances in neural information processing systems, 2012, pp. 1097-1105.

[30] M. M. Lau and K. H. Lim, "Investigation of activation functions in deep belief network," in Control and Robotics Engineering (ICCRE), 2017 2nd International Conference on. IEEE, 2017, pp. 201-206.

[31] Y. LeCun, L. Bottou, Y. Bengio, and P. Haffner, "Gradient-based learning applied to document recognition," Proceedings of the IEEE, vol. 86, no. 11, pp. 2278-2324, 1998.

[32] M. Liang and X. Hu, "Recurrent convolutional neural network for object recognition," in The IEEE Conference on Computer Vision and Pattern Recognition (CVPR), 2015, pp. 3367-3375.

[33] H. Lodish, "Overview of neuron structure and function," Jan 1970. [Online]. Available: https://www.ncbi.nlm.nih.gov/books/NBK21535/

[34] J. Long, E. Shelhamer, and T. Darrell, "Fully convolutional networks for semantic segmentation," in The IEEE Conference on Computer Vision and Pattern Recognition (CVPR), 2015, pp. 3431-3440.

[35] J. L. McClelland and D. E. Rumelhart, Parallel distributed processing: exploration in the microstructure of cognition. The MIT Press, 1987.

[36] W. S. McCulloch and W. Pitts, "A logical calculus of the ideas immanent in nervous activity," The bulletin of mathematical biophysics, vol. 5, no. 4, pp. 115-133, 1943.

[37] S. Y. Min Lin, Qiang Chen, "Network in network," in International Conference on Learning Representations, 2013.

[38] M. Minsky, S. A. Papert, and L. Bottou, Perceptrons: An introduction to computational geometry. MIT press, 2017.

[39] V. Nair and G. E. Hinton, "Rectified linear units improve restricted boltzmann machines," in Proceedings of the 27th international conference on machine learning (ICML-10), 2010, pp. 807-814.

[40] G. Ning, Z. Zhang, C. Huang, X. Ren, H. Wang, C. Cai, and Z. He, "Spatially supervised recurrent convolutional neural networks for visual object tracking," in Int. Symp. on Circuits and Systems (ISCAS). IEEE, 2017, pp. 1-4.

[41] J. Redmon, S. Divvala, R. Girshick, and A. Farhadi, "You only look once: Unified, real-time object detection," in The IEEE Conference on Computer Vision and Pattern Recognition (CVPR), 2016, pp. 779-788. 
[42] S. Ren, K. He, R. Girshick, and J. Sun, "Faster R-CNN: Towards real-time object detection with region proposal networks," in Advances in Neural Information Processing Systems 28, 2015, pp. 91-99.

[43] F. Rosenblatt, "The perceptron: A probabilistic model for information storage and organization in the brain." Psychological review, vol. 65, no. 6, p. 386, 1958.

[44] S. Ruder, "An overview of gradient descent optimization algorithms," arXiv preprint arXiv:1609.04747, 2016.

[45] O. Russakovsky, J. Deng, H. Su, J. Krause, S. Satheesh, S. Ma, Z. Huang, A. Karpathy, A. Khosla, M. Bernstein et al., "Imagenet large scale visual recognition challenge," International Journal of Computer Vision, vol. 115, no. 3, pp. 211-252, 2015.

[46] P. Sermanet, D. Eigen, X. Zhang, M. Mathieu, R. Fergus, and Y. LeCun, "Overfeat: Integrated recognition, localization and detection using convolutional networks," in International Conference on Learning Representations (ICLR 2014). CBLS, 2013, p. 16.

[47] K. Simonyan and A. Zisserman, "Very deep convolutional networks for large-scale image recognition," arXiv preprint arXiv:1409.1556, 2014.

[48] N. Srivastava, G. E. Hinton, A. Krizhevsky, I. Sutskever, and R. Salakhutdinov, "Dropout: a simple way to prevent neural networks from overfitting." Journal of machine learning research, vol. 15, no. 1, pp. 1929-1958, 2014.

[49] H. Su, S. Maji, E. Kalogerakis, and E. Learned-Miller, "Multi-view convolutional neural networks for 3D shape recognition," in The IEEE International Conference on Computer Vision (ICCV), 2015, pp. 945-953.

[50] C. Sun, H. Lu, and M.-H. Yang, "Learning spatial-aware regressions for visual tracking," arXiv preprint arXiv:1706.07457, 2017.

[51] C. Szegedy, W. Liu, Y. Jia, P. Sermanet, S. Reed, D. Anguelov, D. Erhan, V. Vanhoucke, and A. Rabinovich, "Going deeper with convolutions," in Proceedings of the IEEE conference on computer vision and pattern recognition, 2015, pp. 1-9.

[52] C. Szegedy, A. Toshev, and D. Erhan, "Deep neural networks for object detection," in Advances in neural information processing systems, 2013, pp. 25532561.

[53] Tensorflow, "tensorflow/tensorflow-slim," Nov 2017. [Online]. Available: https://github.com/tensorflow/tensorflow/tree/master/tensorflow/contrib/slim

[54] K. E. Van de Sande, J. R. Uijlings, T. Gevers, and A. W. Smeulders, "Segmentation as selective search for object recognition," in Computer Vision (ICCV), 2011 IEEE International Conference on. IEEE, 2011, pp. 1879-1886.

[55] R. Venkatesan, B. Li, and C. Press, Convolutional neural networks in visual computing: a concise guide. Boca Raton;London;: Taylor and Francis, CRC Press, 2017. 
[56] F.-Y. Wang, J. J. Zhang, X. Zheng, X. Wang, Y. Yuan, X. Dai, J. Zhang, and L. Yang, "Where does alphago go: from church-turing thesis to alphago thesis and beyond," IEEE/CAA Journal of Automatica Sinica, vol. 3, no. 2, pp. 113$120,2016$.

[57] P. J. Werbos, "Beyond regression: New tools for prediction and analysis in the behavioral sciences," Doctoral Dissertation, Applied Mathematics, Harvard University, MA, 1974.

[58] F. Yu and V. Koltun, "Multi-scale context aggregation by dilated convolutions," in International Conference on Learning Representations (ICLR), 2015.

[59] M. D. Zeiler and R. Fergus, "Visualizing and understanding convolutional networks," in European conference on computer vision. Springer, 2014, pp. 818-833.

[60] W. Zhang, "Shift-invariant pattern recognition neural network and its optical architecture," in Proceedings of annual conference of the Japan Society of Applied Physics, 1988.

[61] W. Zhang, K. Itoh, J. Tanida, and Y. Ichioka, "Parallel distributed processing model with local space-invariant interconnections and its optical architecture," Applied optics, vol. 29, no. 32, pp. 4790-4797, 1990. 